LOCKHEED MATTIN

ENVIRONMENTAL

RESTORATION

PROGRAM

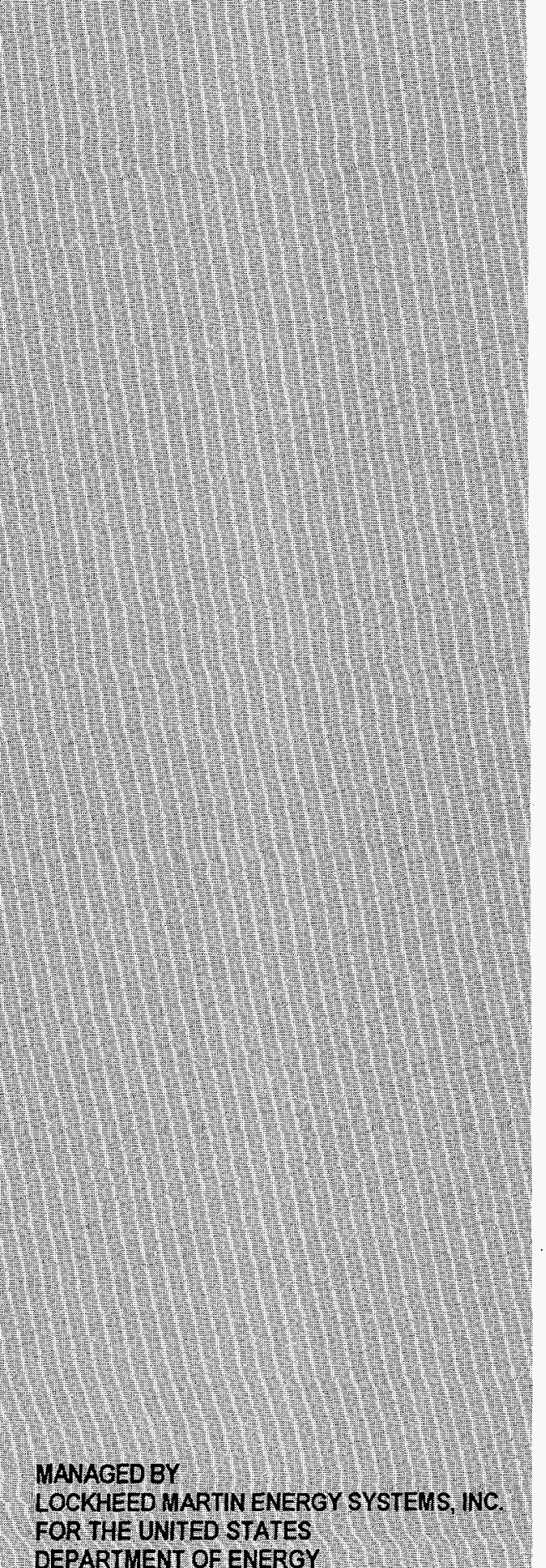

DEPARTMENT OF ENERGY

\title{
Field Characterization Report on Phase I of the Bear Creek Valley Treatability Study, Oak Ridge Y-12 Plant, Oak Ridge, Tennessee
}

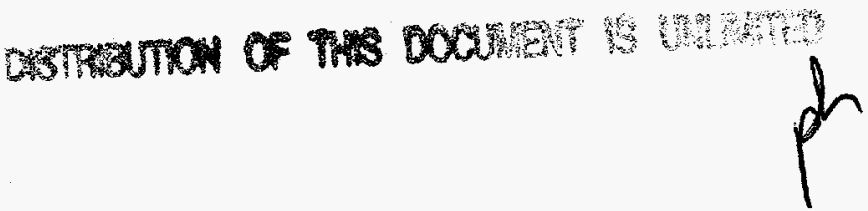

This document has been approved by the Y-12 Plant Technical Information Office for release to the public. Date: 3/31/97

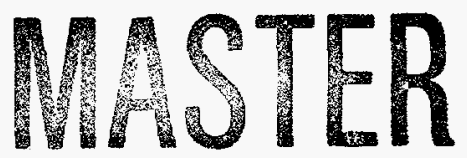

ENERGY SYSTEMS 


\section{Science Applications International Corporation}

contributed to the preparation of this document and should not be considered an eligible contractor for its review. 
Energy Systems Environmental Restoration Program

\section{Field Characterization Report on Phase I of the Bear Creek Valley Treatability Study, Oak Ridge Y-12 Plant, Oak Ridge, Tennessee}

Date Issued-April 1997

Prepared by

Science Applications International Corporation

Oak Ridge, Tennessee

under subcontract 78B-99069C

Prepared for the

U.S. Department of Energy

Office of Environmental Management

under budget and reporting code EW 20

Environmental Management Activities at the

OAK RIDGE Y-12 PLANT

Oak Ridge, Tennessee 37831

managed by

LOCKHEED MARTIN ENERGY SYSTEMS, INC.

for the

U.S. DEPARTMENT OF ENERGY

under contract DE-AC05-84OR21400 



\section{PREFACE}

The Field Characterization Report on Phase I of the Bear Creek Valley Treatability Study, Oak Ridge Y-12 Plant, Oak Ridge, Tennessee (Y/ER-286) was prepared under the Environmental Restoration Program to support the investigation of the practicality of the use of passive, in situ treatment systems to remove contaminants from the Bear Creek Valley Characterization Area. This work was performed under Work Breakdown Structure (WBS) 1.1.02.41.10.34.20. Results from this report will be used to direct further investigation during Phases II and III. 



\section{DISCLAIMER}

This report was prepared as an account of work sponsored by an agency of the United States Government. Neither the United States Government nor any agency thereof, nor any of their employees, make any warranty, express or implied, or assumes any legal liability or responsibility for the accuracy, completeness, or usefulness of any information, apparatus, product, or process disclosed, or represents that its use would not infringe privately owned rights. Reference herein to any specific commercial product, process, or service by trade name, trademark, manufacturer, or otherwise does not necessarily constitute or imply its endorsement, recommendation, or favoring by the United States Government or any agency thereof. The views and opinions of authors expressed herein do not necessarily state or reflect those of the United States Government or any agency thereof. 


\section{CONTENTS}

PREFACE $\ldots \ldots \ldots \ldots \ldots \ldots \ldots \ldots \ldots \ldots \ldots \ldots \ldots \ldots \ldots \ldots \ldots \ldots \ldots$

TABLES $\ldots \ldots \ldots \ldots \ldots \ldots \ldots \ldots \ldots \ldots \ldots \ldots \ldots \ldots \ldots \ldots \ldots \ldots$

FIGURES $\ldots \ldots \ldots \ldots \ldots \ldots \ldots \ldots \ldots \ldots \ldots \ldots \ldots \ldots \ldots \ldots \ldots$

ABBREVIATIONS $\ldots \ldots \ldots \ldots \ldots \ldots \ldots \ldots \ldots \ldots \ldots \ldots \ldots \ldots \ldots \ldots$

EXECUTIVE SUMMARY $\ldots \ldots \ldots \ldots \ldots \ldots \ldots \ldots \ldots \ldots \ldots \ldots \ldots \ldots \ldots$

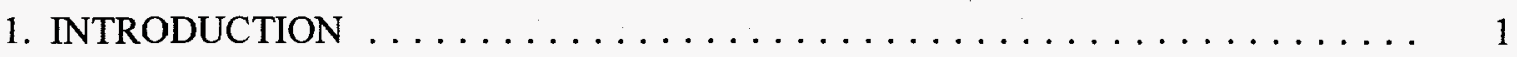

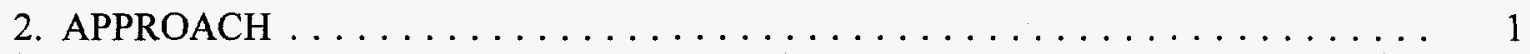

3. SITE CHARACTERIZATION FIELD ACTIVITIES $\ldots \ldots \ldots \ldots \ldots \ldots \ldots \ldots$

3.1 TASK 1 AND TASK 2 SURFACE WATER SAMPLING $\ldots \ldots \ldots \ldots \ldots \ldots$

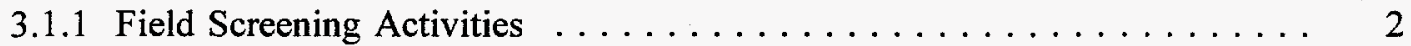

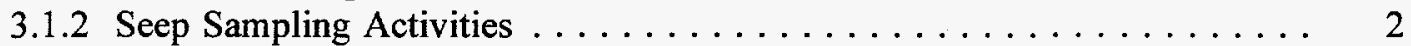

3.1.3 Results of Surface Water Sampling and Analysis . . . . . . . . . . 3

3.2 PIEZOMETER INSTALLATION AND GROUNDWATER SAMPLING $\ldots \ldots$

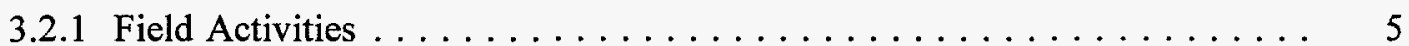

3.2.2 Groundwater Sampling Results . . . . . . . . . . . . . . . 6

3.3 WATER TABLE MONITORING AND HYDRAULIC TESTING $\ldots \ldots \ldots \ldots$

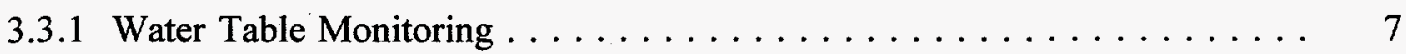

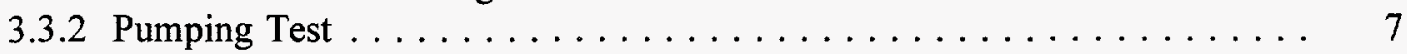

4. CONCLUSIONS $\ldots \ldots \ldots \ldots \ldots \ldots \ldots \ldots \ldots \ldots \ldots \ldots \ldots$

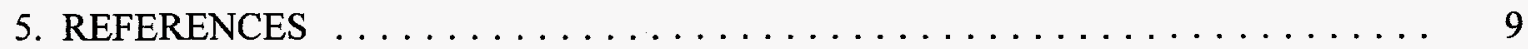

APPENDIX A: WELL DEVELOPMENT REPORTS (GW-834, -835, and -837) . . . . A-1

APPENDIX B: AQUIFER PUMPING TEST RESULTS $\ldots \ldots \ldots \ldots \ldots \ldots \ldots \ldots$

APPENDIX $\mathrm{C}$ : SURVEY COORDINATES $\ldots \ldots \ldots \ldots \ldots \ldots \ldots \ldots \ldots \ldots$ 



\section{TABLES}

1 Bear Creek surface water screening parameters (collected 6/6/96 and 6/14/96) $\ldots \ldots \ldots 13$

2 NT-1 surface water screening parameters (collected 6/4/96) $\ldots \ldots \ldots \ldots \ldots \ldots \ldots \ldots$

3 NT-2 surface water screening parameters (collected 6/5/96) $\ldots \ldots \ldots \ldots \ldots \ldots \ldots \ldots$

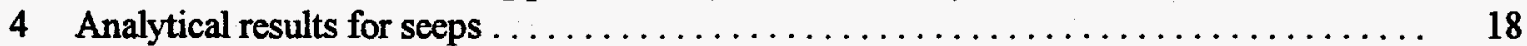

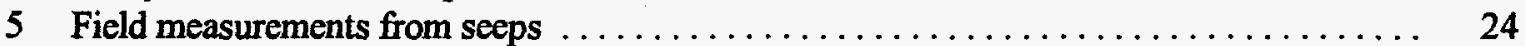

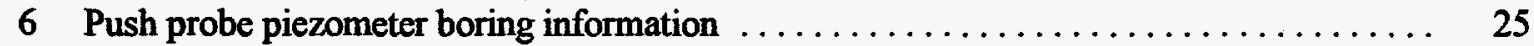

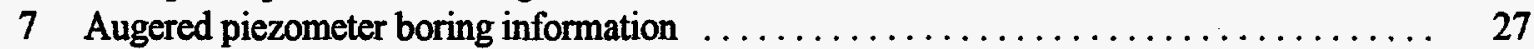

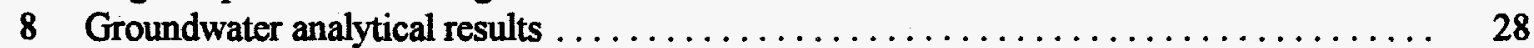

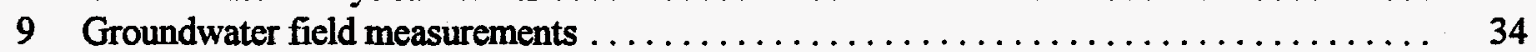

10 Migration pathway summary chemical characteristics $\ldots \ldots \ldots \ldots \ldots \ldots \ldots \ldots \ldots$ 



\section{FIGURES}

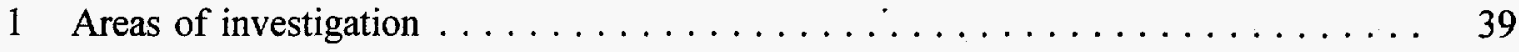

2 Bear Creek surface water sample locations and field screening results . . . . . . 40

3 North Tributary 1 (NT-1) surface water sample locations and field

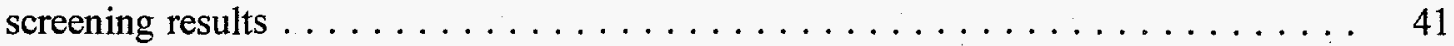

4 North Tributary 2 (NT-2) surface water sample locations and field

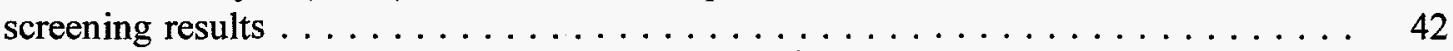

5 Bear Creek surface water uranium screening results $\ldots \ldots \ldots \ldots \ldots \ldots \ldots 43$

6 North Tributary 1 (NT-1) surface water uranium screening results $\ldots \ldots \ldots \ldots .44$

7 North Tributary 2 (NT-2) surface water uranium screening results $\ldots \ldots \ldots \ldots .45$

8 Push probe temporary piezometer locations and groundwater screening results .... 46

9 BCV treatability study $\mathrm{S}-3$ area location map $\ldots \ldots \ldots \ldots \ldots \ldots \ldots \ldots . \ldots \ldots$

10 General piezometer construction $\operatorname{logs} \ldots \ldots \ldots \ldots \ldots \ldots \ldots \ldots \ldots \ldots, 48$

11 Contaminant migration pathways $\ldots \ldots \ldots \ldots \ldots \ldots \ldots \ldots \ldots \ldots \ldots, 49$

12 GW-834 continuous water level, temperature, and conductivity monitoring $\ldots \ldots .50$

13 GW-835 continuous water level, temperature, and conductivity monitoring ..... 51

14 GW-836 continuous water level, temperature, and conductivity monitoring $\ldots \ldots, 52$

15 GW-837 continuous water level, temperature, and conductivity monitoring $\ldots \ldots \ldots 53$

16 BCV treatability study $\mathrm{S}-3$ area water table elevation $(\mathrm{ft}$ amsl) $\ldots \ldots \ldots \ldots \ldots . \ldots 4$ 



\section{ABBREVIATIONS}

BCK

Bear Creek kilometer

BYBY

Boneyard/Burnyard

Energy Systems

Lockheed Martin Energy Systems, Inc.

GWPP

ICP

(Y-12) Groundwater Protection Program

PVC

inductively coupled plasma

TDS

polyvinyl chloride

total dissolved solids

TSS

total suspended solids

VOA

volatile organic analysis 



\section{EXECUTIVE SUMMARY}

A treatability study is being performed to investigate the practicability of using passive, in situ treatment systems to remove contaminants from the Bear Creek Valley (BCV) Characterization Area (CA), as outlined in Bear Creek Valley Characterization Area Technology Demonstration Action Plan (SAIC 1996a). This draft document is a report of the site characterization results and is part of Phase I of this study. Field activities performed are outlined in Bear Creek Valley Passive Surface Water Treatment Technology Demonstrations, Phase I, Site Characterization (SAIC 1996b). The focus of the characterization was to obtain sufficient site-specific data on hydrogeology of NT-1, NT-2, and upper Bear Creek (above its confluence with NT-1) to support selection of groundwater capture and treatment systems in Phases II and III. Groundwater samples from the S-3 Site and NT-1 area were also collected for the principal investigators to test during Phase I laboratory work (SAIC 1996a).

Three contaminant migration pathways were delineated in the S-3 Area. Each is described and briefly characterized by field observations and analysis of surface and groundwater collected within each pathway:

Migration pathway 1: to Bear Creek south of the S-3 site; shown by elevated conductivity, nitrate concentrations, uranium concentrations, and gross alpha and beta activity and by a relatively low $\mathrm{pH}$.

Migration pathway 2: $\quad$ to Bear Creek $-1000 \mathrm{ft}$ southwest of the S-3 site; shown by elevated uranium concentrations and gross alpha and beta activity. Nitrate concentrations were not elevated.

Migration pathway 3: along NT-1 and continuing to NT-2; shown by elevated conductivity, nitrate concentrations, and gross alpha and beta activity. Uranium concentrations are relatively low overall. As this pathway discharges at NT-2 further along stratigraphic strike, it is characterized by elevated conductivity and gross alpha and beta activity. 


\section{INTRODUCTION}

This report describes the results of site characterization activities outlined in Phase I of the Bear Creek Valley Characterization Area Technology Demonstration Action Plan (SAIC 1996a). Detailed direction regarding the Sampling and Analysis Plan, the Quality Assurance Project Plan, the Health and Safety Plan, and the Waste Management Plan for the site characterization is presented in the Bear Creek Valley Passive Surface Water Treatment Technology Demonstrations, Phase I, Site Characterization (SAIC 1996b). The areas of investigation are shown on Fig. 1.

The scope of Phase I site characterization at Bear Creek, NT-1, and NT-2 included six tasks: (1) surface water field screening, (2) surface water and seep sampling, (3) temporary piezometer installation and groundwater sampling, (4) hydraulic testing, (5) data evaluation, and (6) project support and technical report preparation. The detailed activities of each task are defined in the Sampling and Analysis Plan (Sect. 2) of the Bear Creek Valley Passive Surface Water Treatment Technology Demonstrations, Phase I, Site Characterization (SAIC 1996b).

\section{APPROACH}

The investigation approach for the site characterization was designed to determine the following (SAIC 1996b):

- locate the discrete points of groundwater discharge in tributaries NT-1, NT-2, and Bear Creek adjacent to the S-3 Site through surface water sample collection and in-field screening (Task 1);

- determine contaminant geochemistry of surface water and selected seeps along these reaches (Task 2);

- analyze groundwater field parameters near discharge points and identify locations of groundwater contaminant pathways (Task 3);

- provide site-specific groundwater and geotechnical data for the Phase I laboratory testing of potential treatment media (Task 3); and

- obtain sufficient data on site hydrogeology to support Phase I laboratory testing and location of the groundwater capture and treatment systems in Phases II and III (Task 4).

\section{SITE CHARACTERIZATION FIELD ACTIVITIES}

The site characterization field activities included Tasks 1 through 4 . The results of each task are summarized below. 
Surface and groundwater field screen parameters were measured using a Horiba U-10 instrument (specific conductivity, $\mathrm{pH}$, and temperature). Nitrate concentrations were determined in the field using a HACH AccuVac Nitraver 5 Nitrate Test Kit with a measurement range of 0 to $50 \mathrm{ppm}$.

Surface water for analysis was collected as grab samples using a dipper [for volatile organic analysis (VOA) collection] and a peristaltic pump. Use of the pump allowed filtering of samples in the field with disposable in-line filters.

\subsection{TASK 1 AND TASK 2 SURFACE WATER SAMPLING}

\subsubsection{Field Screening Activities}

Surface water grab samples were collected at 73 locations at approximately $50 \mathrm{ft}$. spacing during a summer baseflow period (June 1996) to find discrete points of groundwater discharge along reaches of Bear Creek, NT-1, and NT-2 (Figs. 2 through 4). Sample collection in NT-1 and NT-2 was upstream from the confluence with Bear Creek of each tributary. Sample collection in Bear Creek was conducted upstream of pooled water caused by a beaver dam at Bear Creek kilometer (BCK) 12.46.

Samples were collected from the main channel of each stream and from identifiable seeps adjacent to the main channels. Recognition of seepage areas was done visually (by noting obvious wet areas) and by observing trends, specific conductivity, $\mathrm{pH}$, and temperature in the main stream. Field analysis of nitrate concentration was also performed at each sample location. Trends in the field parameter measurements for NT-1, NT-2, and Bear Creek are shown in Figs. 2 through 4. Selected locations were sampled and analyzed for uranium (Figs. 5 through 7 ). These data are also summarized in Tables 1 through 3.

The upper reach of Bear Creek was initially sampled during a dry (summer low flow) period (June 6, 1996). Except for a small pool located at Station BC 135, the creek was dry upstream of Station BC 1030. Initiation of flow coincided with the locations of two seeps adjacent to the stream channel. Data from the previously dry reach were collected at a later date following a rain event (summer high flow period, June 14, 1996) (Fig. 2).

\subsubsection{Seep Sampling Activities}

Surface water (seep) grab samples were collect from five seep locations (Figs. 2 through 4): Stations BC 1030, BC 1280, NT1 200, NT1 390, and NT2 970. The purpose was to determine surface water contaminant geochemistry in these seeps. The sample locations were at the most active or probably contaminated seeps based on the results of Task 1 field screening. These samples were analyzed for the following: volatile organics, inductively coupled plasma (ICP) metals, radiochemistry (including fluorimetric uranium and tritium), anions, alkalinity, mercury, bicarbonate, and total dissolved solids/total suspended solids (TDS/TSS) (Table 4). Field parameters were also measured at time of collection (Table 5). 


\subsubsection{Results of Surface Water Sampling and Analysis}

Specific conductivity, $\mathrm{pH}$, temperature, and nitrate concentration observed during the screening of surface water and seeps was used to identify discrete points of groundwater discharge at Bear Creek, NT-1, and NT-2. Observations for each stream reach are summarized below.

\section{Bear Creek}

- Eight seeps were identified along Bear Creek: BC 600, BC 900, BC 1030, BC 1150, BC 1215, BC 1280, BC 1300, and BC 1330. During summer baseflow (June 6, 1996), stream flow began at Station 1030 (Fig. 2). Following a period of rain prior to June 14, 1996, water was present in the previously dry reach of Bear Creek upstream of Station BC 1030 (summer high flow) and allowed identification of seeps at BC 600 and BC 900.

- In-stream values for $\mathrm{pH}(7.0$ to 7.4$)$ and temperature $\left(16^{\circ}\right.$ to $\left.19^{\circ} \mathrm{C}\right)$ were similar along the reaches of upper Bear Creek sampled during high and low flow periods. A short reach with significantly higher specific conductivity water was observed between Stations BC 500 and 750 during the high flow period measured on June 14, 1996 (Fig. 2). In-stream values for specific conductance outside this short reach were similar during high and low flow periods $(\sim 1.0 \mathrm{mS} / \mathrm{cm} \mathrm{M})$.

- In-stream nitrate concentrations were 2 to 3 times higher in the low flow period compared to the high flow period.

- Identified seeps upstream of BC 1030 (summer high flow period only) generally had screening values similar to adjacent in-stream values for specific conductance, $\mathrm{pH}$, and temperature. Identified seeps downstream of and including BC 1030 (summer low flow period only) fit two patterns compared with adjacent in-stream values: (1) similar nitrate concentrations and lower temperature (BC 1030, 1150, and 1330) and (2) nondetectable nitrate concentrations and slightly higher temperature (BC 1215, 1280, and 1300). For seeps below $\mathrm{BC} 1030, \mathrm{pH}$ values were generally lower than in-stream values.

- Uranium concentrations in seeps upstream of BC 1030 (high flow period) were 2 to 3 times higher than those seeps downstream of and including BC 1030 (low flow period).

No single seep can account for uranium contamination in upper Bear Creek. Contamination is likely derived from more diffuse seepage of groundwater into the channel of Bear Creek above BCK 12.46. Identifiable seeps may define the discharge points of preferential groundwater flow path(s). The occurrence of a groundwater discharge point adjacent to the location where dry weather flow starts in Bear Creek may indicate that this seepage area (BC 1030) is connected to this pathway(s).

NT-1

- Three active seeps were identified in NT-1: NT1 075, NT1 200, and NT1 390. 
- Seeps at NT-1 are characterized by higher specific conductivity and nitrate concentrations and lower $\mathrm{pH}$ compared to adjacent in-stream values (Fig. 3).

- The in-stream concentration of nitrate increases downstream from $\sim 10 \mathrm{ppm}$ at NT1 500 to $>50 \mathrm{ppm}$ at NT1 250 where the concentration exceeded the upper limit of the in-field analytical method $(50 \mathrm{ppm})$. Concentrations of nitrate at seeps NT1 200 and NT1 390 measured in the laboratory were 1800 and $720 \mathrm{ppm}$, respectively (September 19, 1996). The concentration of nitrate and the specific conductance at seep NT1 390 varied between sampling events (June 4 and September 19, 1996): nitrate concentration increased from 20 to $720 \mathrm{ppm}$ and specific conductance increased from 1.07 to $17.4 \mathrm{mS} / \mathrm{cm}$.

- Coincident with the increase of nitrate concentration, $\mathrm{pH}$ of in-stream flow decreases downstream from NT1 390. This is consistent with the additive discharge of groundwater with lower $\mathrm{pH}$ from the seeps to NT-1 along this reach.

- Conductivity measured while collecting a surface water sample at Station NT1 390 on September 19, 1996, was significantly higher $(17.4 \mathrm{mS} / \mathrm{cm})$ than during the Task 1 field screenings on June 4, $1996(1.07 \mathrm{mS} / \mathrm{cm})$. The only other surface water sample location with higher measured conductivity was Station NT1 $075(22.3 \mathrm{mS} / \mathrm{cm})$.

No single seep can account for contamination observed in NT-1. However, NT-1 is contaminated by discharge from a zone extending from $\sim$ NT1 500 to NT1 50 . Within this zone, identifiable seeps are located at sites with higher flux of groundwater discharge (NT1 75, 200, and 390) and may indicate preferential groundwater flow paths. These seeps probably account for a large proportion of the total contaminant influx into NT-1.

NT-2

- Two active seeps were identified in NT-2: NT2 645 and NT2 970.

- Seeps at NT-2 are characterized by higher conductivity and nitrate concentrations and lower $\mathrm{pH}$ compared to values measured in the adjacent stream (Fig. 4).

- The in-stream nitrate concentration increases from $<20 \mathrm{ppm}$ to $>50 \mathrm{ppm}$ between NT2 1000 and NT2 950. This is coincident with the seep at NT2 970 which had nitrate concentration of $1000 \mathrm{ppm}$ on September 12, 1996.

No single seep can account for contamination observed in NT-2. However, this stream is contaminated by discharge from a zone extending from $\sim$ NT2 645 to NT2 970 . Within this zone, two identifiable seeps are located at sites with higher flux of groundwater discharge (NT2 645 and 970) and indicate preferential groundwater flow paths. Of these two seeps, NT2 970 appears to account for the largest proportion of the total contaminant influx into NT-2. 


\subsection{PIEZOMETER INSTALLATION AND GROUNDWATER SAMPLING}

\subsubsection{Field Activities}

To identify groundwater contaminant pathways, temporary piezometers were installed adjacent to locations of groundwater discharge recognized in the previous tasks. The piezometers were installed in two phases. The first phase involved installation of 25 temporary piezometers (TPB-01, -02, -04 through -16, and -18 through -27) using push-probe (geoprobe) drilling methods (Fig. 8). In addition, two temporary piezometers P1 and P2 were already located close to NT-1 (Fig. 9). All borings were pushed to refusal (bedrock) and piezometers constructed using 1-in. diameter polyvinyl chloride (PVC) casing with 2-ft screened intervals at total depth (Fig. 9). Groundwater grab samples were collected from each piezometer during the sampling event (summer baseflow period) and field screened for specific conductivity, $\mathrm{pH}$, temperature, and nitrate concentration; most piezometers were also sampled for uranium screening. During this phase, a staged approach was employed to use the results from the initial piezometer screenings to determine the locations of the later ones. Boring information for each push-probe temporary piezometer, including depth to water measured in each on June 24, 1996, is in Table 6. Total depth of piezometers in this stage ranges for 5.8 to $23.3 \mathrm{ft}$.

In the second phase, four temporary piezometers (GW-834 through -837) were installed using hollow-stem auger drilling methods (Fig. 9). These temporary piezometers were located to intercept contaminated groundwater flow identified from results for the push-probe installed piezometers. All borings were augered to refusal (bedrock) and piezometers constructed using 4-in. diameter PVC casing with 2-ft screened intervals at total depth (Fig. 10). Attempts were unsuccessful to collect shelby tube samples for geotechnical analysis from these borings. The shelby tubes could not be pushed through the sample interval without crushing them. Each piezometer was developed; however, GW-836 was not completely developed due to slow recharge rate. Boring information for each augered temporary piezometer is in Table 7.

Groundwater grab samples were collected from three of the piezometers (GW-834, -835, and -837) and analyzed for the following: volatile organics, ICP metals, radiochemistry (including fluorimetric uranium and tritium), anions, alkalinity, mercury, bicarbonate, and TDS/TSS (Table 8). Field measurements were also recorded at the time of sample collection (Table 9).

To better delineate groundwater contaminant pathways, five additional temporary piezometers (TPB-28 through -32) were installed at the end of the second phase using push probe (air hammer) techniques (Fig. 8). All borings were pushed to refusal (bedrock) and piezometers constructed using 1-in. diameter PVC with 2.1-ft screened intervals at total depth (Fig. 9). Groundwater grab samples were collected from these piezometers for field screening of specific conductivity, $\mathrm{pH}$, and temperature as well as uranium screening.

Each of the push-probe temporary piezometers were sampled in a single event between June 14 and June 26, 1996. Following a heavy rainstorm, the piezometers at NT-1 were sampled on August 2, 1996 for field screening (specific conductivity, $\mathrm{pH}$, and temperature). The highest specific conductivity reading for groundwater $(30.3 \mathrm{mS} / \mathrm{cm})$ was observed in TPB-02 (Fig. 8).

Elevated beta/gamma readings were encountered during the boring of GW-836 ( $\sim 400 \mathrm{cpm})$. These readings were from soil below the water table brought to the surface on the augers. 
Elevated mercury vapor concentration was measured in the soil cuttings from GW-837 (maximum $0.777 \mathrm{mg} / \mathrm{ml}$ ). These cuttings were left uncovered overnight and mercury vapor concentration dropped below an acceptable value, allowing work to continue.

\subsubsection{Groundwater Sampling Results}

Screening results of groundwater from the temporary push-probe piezometers were consistent with the screening results from the active seeps (groundwater discharge points) recognized during the surface water screenings. By using a staged approach in installing these piezometers, locations of groundwater contaminant pathways during the summer baseflow period have been delineated. Three principal migration pathways have been identified and are shown in Fig. 11 . The screening results from push probe piezometers characterizing these pathways are summarized below:

Migration pathway 1: to Bear Creek south of the S-3 site; shown in TPB-09 and TPB-19 by elevated TDS, nitrate, uranium, and ${ }^{99} \mathrm{Tc}$ concentrations and by a decrease in $\mathrm{pH}$.

Migration pathway 2: $\quad$ to Bear Creek through the area where TPB-07, $-08,-15$, and -16 are located; shown by elevated uranium concentrations. Nitrate concentrations in these piezometers were not elevated.

Migration pathway 3: to NT-1 and extending to NT-2; shown by elevated TDS and nitrate concentrations in TPB-01, $-02,-04$, and-24. Uranium concentrations in this pathway were low. This pathway also discharges to NT-2 further along stratigraphic strike. At NT-2, it is characterized by elevated TDS in Piezometer TPB-12.

Augered piezometers are located within one of the three contaminant pathways recognized along NT-1 and Bear Creek (Fig. 11). An evaluation of the analytical results of groundwater collected from the augered piezometers GW-834, -835 , and -837 shows the values to be consistent with the push-probe piezometers field screening results. The analytical results are summarized in Table 8.

The analytical results from the seep at Station NT1 200 at NT-1 (Fig. 3) may show a significant exception to the characterization of these contaminant pathways. At this location, uranium concentration is similar to that seen in the temporary piezometers within migration pathways 1 and 2 along Bear Creek whereas results from sampling other seeps and piezometers in the NT-1 pathway indicate that uranium was present in very low concentrations $(<10 \mu \mathrm{g} / \mathrm{L})$. The analytical results from the groundwater seep at NT1 200 are summarized below:

- NT1 200 (in migration pathway 3.):

nitrate concentration - $1800 \mathrm{mg} / \mathrm{L}$; uranium concentration $-2.1 \mathrm{mg} / \mathrm{L}$;

gross alpha - $56 \mathrm{pCi} / \mathrm{L}$; gross beta - $740 \mathrm{pCi} / \mathrm{L}$; Technetium-99 - 15,000 pCi/L. 


\subsection{WATER TABLE MONITORING AND HYDRAULIC TESTING}

The purpose of this task was to obtain sufficient data on site hydrogeology to support Phase I laboratory testing and Phases II and III location selection of the groundwater capture and treatment systems (SAIC 1996a).

\subsubsection{Water Table Monitoring}

Continuous monitoring of water level, specific conductivity, and temperature in GW-834, $-835,-836$, and -837 began on August 28, 1996, and continued through September 30, 1996 (one month duration). The results of the monitoring are shown on Figs. 12 through 15 . Water levels in each piezometer showed responses to precipitation; however, neither water temperature nor specific conductance showed any changes that could be related to precipitation events. Both GW-834 and GW-835 showed rapid increases in water level within a few hours of initiation of precipitation. GW-836 and GW-837 showed more subdued responses. These results indicate that although rapid recharge to shallow groundwater at the S-3 site occurs, the volume of recharge for individual events is not sufficient to result in measurable changes in groundwater chemical parameters.

\subsubsection{Pumping Test}

A pumping test was performed on November 12, 1996, and is described in detail in Appendix B. The purpose of the test was to characterize the hydraulics of the shallow, unconsolidated zone groundwater interval at this site and estimate the pumping flow rates that could be sustained in GW-835. Before the test started, water level in all the observation piezometers was measured (Fig. 16). During the test, water levels were measured in the pumped piezometer (GW-835) and in nine adjacent observation piezometers (GW-836 and TPB-06, -07 , $-15,-16,-18,-26,-29$, and -30 ).

The pumping rate during the majority of the test was $\sim 6 \mathrm{~L} / \mathrm{min}$. This rate was the maximum possible for pump with the small water column above the pump intake at test initiation $(\sim 6.5 \mathrm{ft})$. The water level in GW-835 appeared to stabilize and remain relatively constant at this pumping rate.

The purpose of the pumping test was to characterize the unconsolidated groundwater interval and estimate the flow rates that the pumped piezometer could sustain with time. Groundwater grab samples were also collected at the beginning, middle, and near end of the test to determine if there was variation in the chemical composition during the period.

Water level responses in piezometers during the pumping test are shown in Fig. B2. With the exception of TPB-18, no response to the pumping of GW-835 was observed in the piezometers. Much of the fluctuation in water levels could most likely be attributed to normal daily variation in the water table. In TPB-18 drawdown of $\sim 0.35 \mathrm{ft}$ was observed 20 minutes after pumping started. As the test progressed the water level in TPB-18 rose to $-0.15 \mathrm{ft}$ below the starting head. This unusual response may indicate a dual storage system. Water levels in piezometers GW-836 and TPB-07, $-26,-29$, and -30 rose slightly as the test progressed, and these piezometers were apparently not hydraulically connected to GW-835. Based on the results observed, the following conclusions were made (refer to Appendix B for details): 
- GW-835 may be capable of producing groundwater at the rate of up to $6 \mathrm{~L} / \mathrm{min}$ based on the relatively constant water level observed in the piezometer following initial drawdown.

- The screened interval in GW-835 appears to be connected to a highly transmissive zone or interval. The groundwater discharge rate into GW-835 during the test was relatively high and recovery of the water level to pre-test level at termination of the test was very rapid $(-5$ mins $)$.

- GW-835 is completed in an interval that is probably not in direct hydraulic connection with the observation piezometers. This was shown by the lack of significant change in water levels in the observation piezometers during the test.

- There was little chemical variation in the groundwater collected during the test.

Historical information may explain the heterogeneous nature of the unconsolidated zone in the vicinity of the pumping test. Surface water drainage patterns were altered due to activities at the Y-12 Plant and, specifically, construction of the S-3 Ponds (Sutton 1995). Interpretation of preconstruction aerial photographs shows a section of the original stream channel of Bear Creek to be north of the present channel (Fig. 11). The S-3 Ponds appear to have been constructed over this filled stream channel. The stream channel continued to the southwest from this point and through the site of the pumping test. This trend appears to agree with the interpreted trend of contaminant migration pathway 2. The trace of the original channel then intersects the current channel of Bear Creek in the vicinity of stream flow initiation observed during the summer baseflow period.

It is possible that GW-835 was completed within the original stream channel. Associated fluvial lag deposits and/or coarse fill material could provide the observed interval of high hydraulic transmissivity. Confinement of this relatively high transmissive interval within the original stream channel boundaries could also explain the heterogeneous nature and the lack of response in the nearby observation piezometers.

\section{CONCLUSIONS}

Field characterization efforts have delineated three primary pathways for contaminated groundwater at the S-3 Site to discharge to surface water (Fig. 11).

- two pathways for uranium-contaminated groundwater to the main stem of Bear Creek adjacent to the former S-3 Ponds. These two pathways were characterized by 4 in. piezometers and are named the GW-835 and GW-837 sites. Groundwater in the shallow pathway to Bear Creek closest to S-3 Ponds (GW-837) also is contaminated with nitrate, ${ }^{99} \mathrm{Tc}$, and some elevated levels of metals, and groundwater in GW-835 is primarily contaminated with uranium and 
- one deeper along strike flow path for uranium, nitrate, PCE, ${ }^{99} \mathrm{Tc}$, metals, and high TDS contaminated groundwater to NT-1. This deeper along strike flow path extends to NT-2 although, at NT-2, the contaminants are predominately nitrate, elevated TDS, metals, VOCs, and ${ }^{99} \mathrm{Tc}$.

Permeability and groundwater flow rates in these pathways have not been defined; however piezometers located in the two pathways for uranium to Bear Creek showed lower than expected drawdown during well development indicating that these zones may have relatively higher permeability than the surrounding formation. Single well pumping tests have shown that GW-835 can sustain a pumping rate of up to $6 \mathrm{~L} / \mathrm{min}$, whereas $\mathrm{GW}-837$ can only sustain $60 \mathrm{~mL} / \mathrm{min}$, and GW-834 a $100 \mathrm{~mL} / \mathrm{min}$ pumping rate. GW-835 probably intersects a groundwater pathway with relatively high hydraulic conductivity and of limited extent which may be related to the presence of a former stream channel for Bear Creek. This stream channel was apparently filled during construction of the S-3 Ponds and the other facilities in this location and has probably provided a preferential pathway for contaminants to migrate from the S-3 Ponds to Bear Creek.

\section{REFERENCES}

Kimbrough, C.W., L.W. Long, and L.W. McMahon. 1994. Environmental Surveillance Procedures Quality Control Program, Rev. 4, ES/EH/INT-14 (replaces ESH/SUB/8721706/1) Martin Marietta Energy Systems, Inc., Oak Ridge K-25 Site, Oak Ridge, Tennessee.

SAIC (Science Applications International Corporation). 1996a. Bear Creek Valley Characterization Area Technology Demonstration Action Plan, Draft, Y/EN-5479, prepared for Lockheed Martin Energy Systems, Inc., Oak Ridge Y-12 Plant, Oak Ridge, Tennessee, April.

SAIC. 1996b. Bear Creek Valley Passive Water Treatment Technology Demonstration, Phase I, Site Characterization, Draft, Y/ER-269, prepared for Lockheed Martin Energy Systems, Inc., Oak Ridge Y-12 Plant, Oak Ridge, Tennessee, June.

Sutton, G.E. 1995. "Distribution of Anthropogenic Fill Material Within Y-12 Plant Area, Oak Ridge, Tennessee," Y-TS-1387. 

TABLES 



\begin{tabular}{|c|c|c|c|c|c|c|c|c|}
\hline Locations & Sample no. & $\begin{array}{l}\text { Conductivity } \\
(\mathrm{mS} / \mathrm{cm})\end{array}$ & $\begin{array}{l}\mathrm{pH} \\
\text { (su) }\end{array}$ & $\begin{array}{l}\text { Temperature } \\
\text { (C) }\end{array}$ & $\begin{array}{l}\text { Nitrate concentration } \\
\text { (ppm) }\end{array}$ & $\begin{array}{l}\text { Gross alpha } \\
(\mathrm{pCi} / \mathrm{L})\end{array}$ & $\begin{array}{l}\text { Gross beta } \\
(\mathrm{pCi} / \mathrm{L})\end{array}$ & $\begin{array}{l}\text { U. Fluor } \\
\text { (ppm) }\end{array}$ \\
\hline \multicolumn{9}{|c|}{ Stream } \\
\hline BC 1430 & $\mathrm{BC} 1213$ & 1.030 & 7.10 & 17.5 & 9 & & & \\
\hline BC 1350 & $\mathrm{BC} 1212$ & 1.020 & 7.14 & 17.7 & 9 & & & \\
\hline BC 1300 & $\mathrm{BC} 1209$ & 1.010 & 7.13 & 17.8 & 8 & & & \\
\hline BC 1250 & $\mathrm{BC} 1207$ & 1.020 & 7.07 & 17.5 & 8 & & & \\
\hline BC 1200 & BC1205 & 1.020 & 7.16 & 17.1 & 7 & & & \\
\hline BC 1150 & BC1203 & 1.050 & 7.06 & 16.3 & 7 & & & \\
\hline BC 1100 & Dry & --- & $\cdots$ & --- & --- & & & \\
\hline BC 1050 & Dry & -- & -- & --- & --- & & & \\
\hline BC 1000 & BC1214* & 1.000 & 7.40 & 18.0 & 3 & & & \\
\hline BC 950 & BC1215* & 1.000 & 7.40 & 18.0 & 3 & & & \\
\hline BC 900 & BC1216* & 0.990 & 7.20 & 17.9 & 3 & 133 & 53.3 & 0.43 \\
\hline BC 850 & BC1217 & 0.493 & 7.01 & 18.1 & 2 & & & \\
\hline BC 800 & BC1218* & 0.990 & 7.01 & 18.9 & 3 & & & \\
\hline BC 750 & $\mathrm{BC} 1219 *$ & 1.770 & 6.99 & 18.7 & 3 & & & \\
\hline BC 700 & $\mathrm{BC} 1220^{*}$ & 1.930 & 7.09 & 18.5 & 2 & & & \\
\hline BC 630 & $\mathrm{BC} 1221^{*}$ & 1.970 & 6.69 & 18.4 & 4 & 149 & 34.4 & 0.49 \\
\hline BC 600 & BC1222* & 1.140 & 7.09 & 18.5 & 3 & & & \\
\hline BC 550 & BC1223* & 2.680 & 7.13 & 18.9 & 0 & & & \\
\hline BC 500 & BC1224* & 0.633 & 7.15 & 19.1 & 3 & 126 & 44.9 & 0.43 \\
\hline
\end{tabular}




\begin{tabular}{|c|c|c|c|c|c|c|c|c|}
\hline Locations & Sample no. & $\begin{array}{l}\text { Conductivity } \\
(\mathrm{mS} / \mathrm{cm})\end{array}$ & $\begin{array}{l}\mathrm{pH} \\
\text { (su) }\end{array}$ & $\begin{array}{l}\text { Temperature } \\
\text { (C) }\end{array}$ & $\begin{array}{l}\text { Nitrate concentration } \\
(\mathrm{ppm})\end{array}$ & $\begin{array}{l}\text { Gross alpha } \\
(\mathrm{pCi} / \mathrm{L})\end{array}$ & $\begin{array}{l}\text { Gross beta } \\
(\mathrm{pCi} / \mathrm{L})\end{array}$ & $\begin{array}{l}\text { U. Fluor } \\
\text { (ppm) }\end{array}$ \\
\hline BC 450 & BC1225* & 0.630 & 7.17 & 19.0 & 3 & & & \\
\hline BC 400 & BC1226* & 0.606 & 7.21 & 19.8 & 3 & & & \\
\hline BC 350 & BC1230* & 0.585 & 7.23 & 20.0 & 2 & & & \\
\hline BC 300 & $\mathrm{BC} 1227^{*}$ & 0.550 & 7.20 & 20.3 & 3 & 67.4 & 30.2 & 0.27 \\
\hline BC 250 & $\mathrm{BC} 1228 *$ & 0.530 & 7.11 & 20.9 & 4 & & & \\
\hline BC 200 & Dry & --- & -- & --- & --- & & & \\
\hline BC 150 & Dry & --- & --- & - & --- & & & \\
\hline BC 135 & BC1229* & 0.501 & 7.09 & 20.5 & 4 & & & \\
\hline \multicolumn{9}{|c|}{ Seep } \\
\hline BC 1330 & $\mathrm{BC} 1211$ & 0.851 & 6.97 & 15.5 & 8 & 65 & 44 & 0.25 \\
\hline BC 1300 & BC1210 & 1.100 & 6.96 & 18.4 & 0 & 52 & 23 & 0.26 \\
\hline BC 1280 & BC1208 & 0.631 & 6.93 & 18.4 & 0 & 47 & 19 & 0.13 \\
\hline BC 1215 & BC1206 & 0.679 & 6.84 & 19.7 & 0 & 46 & 16 & 0.12 \\
\hline BC 1150 & BC1204 & 0.930 & 7.06 & 15.1 & 3 & 79 & 62 & 0.27 \\
\hline BC 1030 & BC1202 & 1.080 & 6.70 & 14.3 & 3 & 80 & 38 & 0.48 \\
\hline BC 900 & BC1216* & 0.990 & 7.20 & 17.9 & 3 & 133 & 53.3 & 0.43 \\
\hline
\end{tabular}




\begin{tabular}{|c|c|c|c|c|c|c|c|c|}
\hline Locations & Sample no. & $\begin{array}{l}\text { Conductivity } \\
(\mathrm{mS} / \mathrm{cm})\end{array}$ & $\begin{array}{l}\mathrm{pH} \\
(\mathrm{su})\end{array}$ & $\begin{array}{l}\text { Temperature } \\
\text { (C) }\end{array}$ & $\begin{array}{c}\text { Nitrate concentration } \\
\text { (ppm) }\end{array}$ & $\begin{array}{l}\text { Gross alpha } \\
(\mathrm{pCi} / \mathrm{L})\end{array}$ & $\begin{array}{c}\text { Gross beta } \\
(\mathrm{pCi} / \mathrm{L})\end{array}$ & $\begin{array}{l}\text { U.Fluor } \\
\text { (ppm) }\end{array}$ \\
\hline \multicolumn{9}{|c|}{ Stream } \\
\hline NT-1 000 & N11201 & 1.370 & 6.84 & 22.1 & 50 & & & \\
\hline NT-1 050 & N11202 & 1.750 & 6.54 & 20.6 & 50 & & & \\
\hline NT-1 100 & N11203 & 1.640 & 6.56 & 20,3 & 50 & & & \\
\hline NT-1 150 & N11205 & 1.470 & 6.68 & 20.3 & 50 & & & \\
\hline NT-1 200 & N11206 & 1.240 & 6.66 & 20.5 & 50 & & & \\
\hline NT-1 250 & N11208 & 1.180 & 6.89 & 20.5 & 49 & & & \\
\hline NT-1 300 & N11209 & 0.960 & 6.91 & 20.4 & 42 & & & \\
\hline NT-1 350 & N11210 & 0.680 & 5.83 & 20.3 & 30 & 6.6 & 37 & 0.004 \\
\hline NT-1 400 & N11211 & 0.484 & 7.49 & 20.8 & 20 & & & \\
\hline NT-1 450 & N11212 & 0.436 & 7.51 & 21.0 & 18 & & & \\
\hline NT-1 500 & $\mathrm{~N} 11213$ & 0.363 & 7.71 & 20.5 & 12 & & & \\
\hline NT-1 550 & N11214 & 0.252 & 7.71 & 19.4 & 5 & & & \\
\hline NT-1 575 & N11215 & 0.244 & 7.58 & 18.9 & 4 & & & \\
\hline \multicolumn{9}{|c|}{ Seep } \\
\hline NT-1 075 & N11204 & 22.300 & 6.08 & 20.5 & 50 & 83 & 910 & 0.016 \\
\hline NT-1 200 & N11207 & 4.410 & 6.01 & 19.5 & 50 & 42 & 780 & 0.0017 \\
\hline NT-1 390 & N11216 & 1.070 & 6.83 & 17.8 & 20 & 6.8 & 100 & 0.0049 \\
\hline
\end{tabular}




\begin{tabular}{|c|c|c|c|c|c|c|c|c|}
\hline Locations & Sample no. & $\begin{array}{l}\text { Conductivity } \\
(\mathrm{mS} / \mathrm{cm})\end{array}$ & $\begin{array}{l}\mathrm{pH} \\
(\mathrm{su})\end{array}$ & $\begin{array}{l}\text { Temperature } \\
\text { (C) }\end{array}$ & $\begin{array}{l}\text { Nitrate concentration } \\
\text { (ppm) }\end{array}$ & $\begin{array}{l}\text { Gross alpha } \\
(\mathrm{pCi} / \mathrm{L})\end{array}$ & $\begin{array}{c}\text { Gross beta } \\
(\mathrm{pCi} / \mathrm{L})\end{array}$ & $\begin{array}{c}\text { U. Fluor } \\
\text { (ppm) }\end{array}$ \\
\hline \multicolumn{9}{|c|}{ Stream } \\
\hline NT-2 000 & $\mathrm{~N} 21201$ & 0.704 & 7.43 & 17.8 & 45 & & & \\
\hline NT-2 050 & N21202 & 0.701 & 7.53 & 17.5 & 41 & & & \\
\hline NT-2 100 & N21203 & 0.697 & 7.59 & 17.0 & 46 & & & \\
\hline NT-2 150 & N21204 & 0.695 & 7.60 & 16.9 & 50 & & & \\
\hline NT-2 200 & N21205 & 0.695 & 7.59 & 16.7 & 49 & & & \\
\hline NT-2 250 & N21206 & 0.695 & 7.53 & 16.4 & 48 & & & \\
\hline NT-2 300 & $\mathrm{~N} 21207$ & 0.695 & 7.55 & 16.4 & 46 & & & \\
\hline NT-2 350 & N21208 & 0.694 & 7.52 & 16.1 & 50 & & & \\
\hline NT-2 400 & N21209 & 0.694 & 7.51 & 16.0 & 47 & & & \\
\hline NT-2 450 & N21210 & 0.694 & 7.46 & 16.0 & 50 & & & \\
\hline NT-2 500 & N21211 & 0.682 & 7.43 & 15.9 & 50 & & & \\
\hline NT-2 550 & N21212 & 0.687 & 7.38 & 16.0 & 49 & & & \\
\hline NT-2 600 & $\mathrm{~N} 21213$ & 0.695 & 7.43 & 16.2 & 50 & & & \\
\hline NT-2 650 & N21214 & 0.696 & 7.44 & 18.9 & 46 & & & \\
\hline NT-2 700 & $\mathrm{~N} 21215$ & 0.726 & 7.47 & 19.0 & 50 & & & \\
\hline NT-2 750 & N21216 & 0.710 & 7.32 & 18.6 & 50 & & & \\
\hline NT-2 800 & N21217 & 0.721 & 7.23 & 18.5 & 50 & & & \\
\hline NT-2 850 & N21218 & 0.672 & 7.07 & 18.6 & 50 & & & \\
\hline NT-2 900 & N21219 & 0.655 & 7.04 & 19.1 & 48 & & & \\
\hline
\end{tabular}




\begin{tabular}{|c|c|c|c|c|c|c|c|c|}
\hline \multicolumn{9}{|c|}{ Table 3 (continued) } \\
\hline Locations & Sample no. & $\begin{array}{l}\text { Conductivity } \\
(\mathrm{mS} / \mathrm{cm})\end{array}$ & $\begin{array}{l}\mathrm{pH} \\
\text { (su) }\end{array}$ & $\begin{array}{l}\text { Temperature } \\
\text { (C) }\end{array}$ & $\begin{array}{l}\text { Nitrate concentration } \\
(\mathrm{ppm})\end{array}$ & $\begin{array}{l}\text { Gross alpha } \\
(\mathrm{pCi} / \mathrm{L})\end{array}$ & $\begin{array}{c}\text { Gross beta } \\
(\mathrm{pCi} / \mathrm{L})\end{array}$ & $\begin{array}{l}\text { U.Fluor } \\
\text { (ppm) }\end{array}$ \\
\hline NT-2 950 & $\mathrm{~N} 21220$ & 0.590 & 6.91 & 19.2 & 50 & & & \\
\hline NT-2 1000 & N21221 & 0.141 & 7.38 & 19.2 & 17 & & & \\
\hline NT-2 1120 & $\mathrm{~N} 21225$ & 0.121 & 7.34 & 18.3 & & & & \\
\hline \multicolumn{9}{|c|}{ Seep } \\
\hline NT-2 645 & $\mathrm{~N} 21224$ & 3.23 & 7.08 & 18.6 & 50 & 11 & 33 & 0.0023 \\
\hline NT-2 970 & N21223 & 12.6 & 6.05 & 15.5 & 50 & 49 & 680 & 0.0015 \\
\hline
\end{tabular}




\begin{tabular}{|c|c|c|c|c|c|c|c|c|c|c|}
\hline \multirow[t]{2}{*}{$\begin{array}{l}\text { Sample no. (location) } \\
\text { Collection date }\end{array}$} & \multicolumn{2}{|c|}{$\begin{array}{c}\text { BC1101 } \\
\text { (BC 1030) } \\
9 / 12 / 96\end{array}$} & \multicolumn{2}{|c|}{$\begin{array}{c}\text { BC1102 } \\
\text { (BC 1280) } \\
9 / 20 / 96\end{array}$} & \multicolumn{2}{|c|}{$\begin{array}{c}\text { NT1101 } \\
\text { (NT1 200) } \\
9 / 19 / 96\end{array}$} & \multicolumn{2}{|c|}{$\begin{array}{c}\text { NT1102 } \\
\text { (NT1 390) } \\
9 / 19 / 96\end{array}$} & \multicolumn{2}{|c|}{$\begin{array}{c}\text { NT2101 } \\
\text { (NT2 970) } \\
\text { 9/12/96 }\end{array}$} \\
\hline & Filtered & Unfiltered & Filtered & Unfiltered & Filtered & Unfiltered & Filtered & Unfiltered & Filtered & Unfiltered \\
\hline \multicolumn{11}{|c|}{ Analysis } \\
\hline Bicarbonate, $\mathrm{mg} / \mathrm{L}$ & & 384 & & 550 & & 30 & & 412 & & 253 \\
\hline Mercury, mg/L & $<0.0002$ & & 0.0006 & & 0.0045 & & $<0.0002$ & & $\begin{array}{l}<0.000 \\
2\end{array}$ & \\
\hline Aluminum, $\mu \mathrm{g} / \mathrm{L}$ & $<200$ & $<200$ & $<200$ & 1530 & 723 & 110000 & 707 & 7210 & $<200$ & 5170 \\
\hline Boron, $\mu \mathrm{g} / \mathrm{L}$ & 62.3 & 5206 & 94.9 & 99.3 & 49.7 & 420 & $<20$ & 63.3 & $<20$ & 55.3 \\
\hline Barium, $\mu \mathrm{g} / \mathrm{L}$ & 72.1 & 72.7 & 285 & 311 & 728 & 958 & 4340 & 4910 & 4290 & 11000 \\
\hline Calcium, $\mu \mathrm{g} / \mathrm{L}$ & 153000 & 153000 & 164000 & 170000 & 360000 & $>1000000$ & 1100000 & 1100000 & 1120 & $>1000000$ \\
\hline Cadmium, $\mu \mathrm{g} / \mathrm{L}$ & $<20$ & $<20$ & $<20$ & $<20$ & 80.7 & 846 & $<20$ & 252 & $<20$ & 590 \\
\hline Cobalt, $\mu \mathrm{g} / \mathrm{L}$ & $<20$ & $<20$ & $<20$ & $<20$ & 35.2 & 601 & $<20$ & 71.8 & $<20$ & 171 \\
\hline Chromium, $\mu \mathrm{g} / \mathrm{L}$ & $<20$ & $<20$ & $<20$ & 69 & $<20$ & $<20$ & $<20$ & $<20$ & $<20$ & $<20$ \\
\hline Copper, $\mu \mathrm{g} / \mathrm{L}$ & $<30$ & $<30$ & $<30$ & $<30$ & $<30$ & $<300$ & $<300$ & $<300$ & $<300$ & $<300$ \\
\hline Iron, $\mu \mathrm{g} / \mathrm{L}$ & $<200$ & $<200$ & 1610 & 5150 & $<200$ & 152 & 1000 & 3790 & $<200$ & $<200$ \\
\hline Potassium, $\mu \mathrm{g} / \mathrm{L}$ & 3820 & 3870 & 6150 & 6560 & 7730 & 58400 & 4960 & 14900 & 4820 & 23800 \\
\hline Magnesium, $\mu \mathrm{g} / \mathrm{L}$ & 21100 & 21000 & 23200 & 23900 & 52200 & 253000 & 139000 & 144000 & 139000 & 241000 \\
\hline Manganese, $\mu \mathrm{g} / \mathrm{L}$ & 951 & 984 & 5050 & 5180 & 11000 & 100000 & 5470 & 52400 & 5060 & 100000 \\
\hline Molybdenum, $\mu \mathrm{g} / \mathrm{L}$ & $<40$ & $<40$ & $<40$ & $<40$ & $<40$ & $<40$ & $<40$ & $<40$ & $<40$ & $<40$ \\
\hline Sodium, $\mu \mathrm{g} / \mathrm{L}$ & 54100 & 53900 & 9890 & 10000 & 50300 & 355000 & 92500 & 147000 & 92000 & 261000 \\
\hline Nickel, $\mu \mathrm{g} / \mathrm{L}$ & $<40$ & $<40$ & $<40$ & $<40$ & 339 & 4930 & $<40$ & 434 & $<40$ & 1030 \\
\hline Lead, $\mu \mathrm{g} / \mathrm{L}$ & $<200$ & $<200$ & $<200$ & $<200$ & $<200$ & $<200$ & $<200$ & $<200$ & $<200$ & $<200$ \\
\hline
\end{tabular}


Table 4 (continued)

\begin{tabular}{|c|c|c|c|c|c|c|c|c|c|c|}
\hline \multirow[t]{2}{*}{$\begin{array}{l}\text { Sample no. (location) } \\
\text { Collection date }\end{array}$} & \multicolumn{2}{|c|}{$\begin{array}{c}\text { BC1101 } \\
\text { (BC 1030) } \\
9 / 12 / 96\end{array}$} & \multicolumn{2}{|c|}{$\begin{array}{c}\text { BC1102 } \\
\text { (BC 1280) } \\
9 / 20 / 96\end{array}$} & \multicolumn{2}{|c|}{$\begin{array}{c}\text { NT1101 } \\
\text { (NT1 200) } \\
9 / 19 / 96\end{array}$} & \multicolumn{2}{|c|}{$\begin{array}{c}\text { NT1102 } \\
\text { (NT1 390) } \\
9 / 19 / 96\end{array}$} & \multicolumn{2}{|c|}{$\begin{array}{c}\text { NT2101 } \\
\text { (NT2 970) } \\
9 / 12 / 96\end{array}$} \\
\hline & Filtered & Unfiltered & Filtered & Unfiltered & Filtered & Unfiltered & Filtered & Unfiltered & Filtered & Unfiltered \\
\hline Thallium, $\mu \mathrm{g} / \mathrm{L}$ & $<100$ & $<100$ & $<100$ & $<100$ & $<100$ & 102 & $<100$ & $<100$ & $<100$ & 114 \\
\hline Vanadium, $\mu \mathrm{g} / \mathrm{L}$ & $<10$ & $<10$ & $<10$ & $<10$ & $<10$ & $<10$ & $<10$ & $<10$ & $<10$ & $<10$ \\
\hline Zinc, $\mu \mathrm{g} / \mathrm{L}$ & 27.2 & $<20$ & 23.8 & 55.6 & 22.7 & 172 & $<20$ & 98.8 & $<20$ & 109 \\
\hline Selenium, $\mu \mathrm{g} / \mathrm{L}$ & $<500$ & $<500$ & $<500$ & $<500$ & $<500$ & $<1000$ & $<500$ & $<500$ & $<500$ & $<1000$ \\
\hline Silver, $\mu \mathrm{g} / \mathrm{L}$ & 24.9 & $<20$ & $<20$ & $<20$ & $<20$ & $<20$ & $<20$ & $<20$ & $<20$ & $<20$ \\
\hline Arsenic, $\mu \mathrm{g} / \mathrm{L}$ & $<100$ & $<100$ & $<100$ & $<100$ & $<100$ & $<100$ & $<100$ & $<100$ & $<100$ & $<100$ \\
\hline Beryllium, $\mu \mathrm{g} / \mathrm{L}$ & $<2$ & $<2$ & $<2$ & $<2$ & $<2$ & 35.7 & $<1.0$ & $<10$ & $<2$ & $<10$ \\
\hline Antimony, $\mu \mathrm{g} / \mathrm{L}$ & $<100$ & $<100$ & $<100$ & $<100$ & $<100$ & $<100$ & $<100$ & $<100$ & $<100$ & $<100$ \\
\hline Lithium, $\mu \mathrm{g} / \mathrm{L}$ & $<10$ & $<10$ & 174 & 199 & $<10$ & 14.1 & 53.9 & 13.1 & 52.9 & $<10$ \\
\hline \multicolumn{11}{|l|}{ Phosphorous, $\mu \mathrm{g} / \mathrm{L}$} \\
\hline Titanium, $\mu \mathrm{g} / \mathrm{L}$ & $<20$ & $<20$ & $<20$ & 16.7 & $<20$ & $<20$ & $<20$ & 49.3 & $<20$ & $<20$ \\
\hline Strontium, $\mu \mathrm{g} / \mathrm{L}$ & 333 & 327 & 709 & 723 & 1010 & 3520 & 4150 & 2910 & 4120 & 4730 \\
\hline Alkalinity, mg/L & & 400 & & 550 & & 30 & & 410 & & 260 \\
\hline Alpha activity, $\mathrm{pC} / \mathrm{L}$ & & $89 \pm 14$ & & $16 \pm 4.9$ & & $56 \pm 11$ & & $170 \pm 35$ & & $63 \pm 16$ \\
\hline Beta activity, $\mathrm{pC} / \mathrm{L}$ & & $78 \pm 12$ & & $10 \pm 4.8$ & & $740 \pm 85$ & & $1700 \pm 200$ & & $850 \pm 100$ \\
\hline Tritium, pC/L & & $170 \pm 580$ & & $3130 \pm 240$ & & $1300 \pm 270$ & & $370 \pm 230$ & & $370 \pm 230$ \\
\hline Fluoride, $\mathrm{mg} / \mathrm{L}$ & & 0.902 & & 0.65 & & 57 & & 2.1 & & \\
\hline Chloride, $\mathrm{mg} / \mathrm{L}$ & & 42 & & 9.5 & & 180 & & 60 & & 58 \\
\hline Nitrite as Nitrogen, $\mathrm{mg} / \mathrm{L}$ & & & & $<0.05$ & & 5.8 & & & & \\
\hline
\end{tabular}


Table 4 (continued)

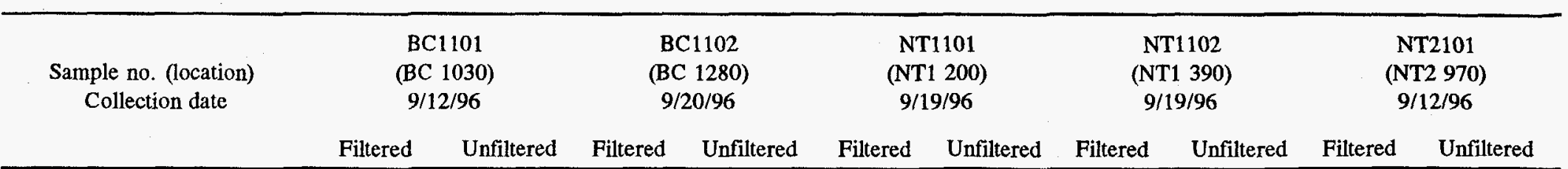

Bromide, mg/L

Nitrate as Nitrogen, $\mathrm{mg} / \mathrm{L}$

Ortho-Phosphate as

Phosphorus, mg/L

Sulfate, $\mathrm{mg} / \mathrm{L}$

22-Sodium Activity, pC/L

40-Potassium Activity, $\mathrm{pC} / \mathrm{L}$

51-Chromium Activity, pC/L

54-Manganese Activity, pC/L

57-Cobalt Activity, pC/L

58-Cobalt Activity, pC/L

59-Iron Activity, pC/L

60-Cobalt Activity, pC/L

65-Zinc Activity, pC/L

95-Niobium Activity, pC/L

95-Zirconium Activity, pC/L

106-Ruthenium Activity,

$\mathrm{pC} / \mathrm{L}$

125-Antimony Activity, pC/L

134-Cesium Activity, pC/L
4.6

4.9

$<0.12$

95

$1.1 \pm 1.6$

$5.6 \pm 4.4$

$1.3 \pm 18$

$0.5 \pm 1.7$

$-0.049 \pm 1.4$

$0.17 \pm 1.8$

$1.8 \pm 4.2$

$0.53 \pm 1.9$

$1.8 \pm 4.1$

$0.53 \pm 2.1$

$-0.75 \pm 4.9$

$7.7 \pm 16$

$-5.3 \pm 4.7$

$-0.33 \pm 1.9$
1800

120

$-0.53 \pm 1.7$

$82 \pm 45$

$-4.3 \pm 18$

$0.18 \pm 1.7$

$0.91 \pm 1.4$

$-0.67 \pm 1.8$

$-0.75 \pm 3.5$

$2.1 \pm 1.8$

$-0.17 \pm 4.3$

$0.85 \pm 2.1$

$-2.3 \pm 4.0$

$-5.3 \pm 15$

$-3.5 \pm 4.7$

$0.44 \pm 1.9$
720

59

$0.53 \pm 1.6$

$4 \pm 39$

$6 \pm 16$

$-0.51 \pm 1.6$

$0.099 \pm 1.2$

$-0.32 \pm 1.6$

$2.6 \pm 3.5$

$0.23 \pm 1.7$

$-1.6 \pm 4.2$

$-0.003 \pm 2.0$

$-5.5 \pm 4.1$

$-2.1 \pm 14$

$3.9 \pm 4.3$

$0.17 \pm 1.8$
1000

3.4

$-0.76 \pm 2.0$

$39 \pm 52$

$1.7 \pm 19$

$-0.52 \pm 1.8$

$-0.35 \pm 1.2$

$0.59 \pm 2.1$

$-3.4 \pm 4.8$

$1.9 \pm 2.1$

$3.8 \pm 5.0$

$2.4 \pm 2.6$

$-2.5 \pm 5.0$

$-18 \pm 17$

$0.41 \pm 4.7$

$-2.5 \pm 2.4$ 


\begin{tabular}{|c|c|c|c|c|c|c|c|c|c|c|}
\hline \multirow[t]{2}{*}{$\begin{array}{l}\text { Sample no. (location) } \\
\text { Collection date }\end{array}$} & \multicolumn{2}{|c|}{$\begin{array}{c}\text { BC1101 } \\
\text { (BC 1030) } \\
9 / 12 / 96\end{array}$} & \multicolumn{2}{|c|}{$\begin{array}{c}\text { BC1102 } \\
\text { (BC 1280) } \\
9 / 20 / 96\end{array}$} & \multicolumn{2}{|c|}{$\begin{array}{c}\text { NT1101 } \\
\text { (NT1 200) } \\
9 / 19 / 96\end{array}$} & \multicolumn{2}{|c|}{$\begin{array}{c}\text { NT1102 } \\
\text { (NT1 390) } \\
9 / 19 / 96\end{array}$} & \multicolumn{2}{|c|}{$\begin{array}{c}\text { NT2101 } \\
\text { (NT2 970) } \\
9 / 12 / 96\end{array}$} \\
\hline & Filtered & Unfiltered & Filtered & Unfiltered & Filtered & Unfiltered & Filtered & Unfiltered & Filtered & Unfiltered \\
\hline 137-Cesium Activity, $\mathrm{pC} / \mathrm{L}$ & & $1.4 \pm 1.8$ & & $4.5 \pm 5.6$ & & $0.52 \pm 1.8$ & & $-0.61 \pm 1.6$ & & $0.005 \pm 2.0$ \\
\hline 144-Cerium Activity, $\mathrm{pC} / \mathrm{L}$ & & $11 \pm 11$ & & $-9.1 \pm 13$ & & $-9.7 \pm 12$ & & $-7.2 \pm 9.1$ & & $-0.43 \pm 9.6$ \\
\hline 208-Thallium Activity, pC/L & & $-1.4 \pm 3.4$ & & $1.9 \pm 3.9$ & & $1 \pm 3.1$ & & $0.53 \pm 3.4$ & & $0.9 \pm 4.3$ \\
\hline 212-Bismuth Activity, $\mathrm{pC} / \mathrm{L}$ & & $24 \pm 19$ & & $2.8 \pm 18$ & & $13 \pm 13$ & & $13 \pm 13$ & & $14 \pm 16$ \\
\hline 212-Lead Activity, pC/L & & $1.8 \pm 5.5$ & & $-4.8 \pm 6.2$ & & $4 \pm 5.6$ & & $0.19 \pm 3.9$ & & $1.3 \pm 5.4$ \\
\hline 235 Uranium Activity, $\mathrm{pC} / \mathrm{L}$ & & $3.3 \pm 4.6$ & & $-14 \pm 14$ & & $13 \pm 4.7$ & & $2.6 \pm 3.9$ & & $16 \pm 17$ \\
\hline Technetium -99, pC/L & & $17 \pm 3.8$ & & $-2 \pm 2.0$ & . & $\begin{array}{l}15000 \\
\pm 1500\end{array}$ & & $\begin{array}{l}5700 \\
\pm 570\end{array}$ & & $\begin{array}{l}1900 \\
\pm 190\end{array}$ \\
\hline Total Dissolved Solids, mg/L & & & & 620 & & 12000 & & 10000 & & \\
\hline Total Strontium, $\mathbf{p C} / \mathbf{L}$ & & $0.14 \pm 0.80$ & & $-0.08 \pm 0.62$ & & $6.3 \pm 1.0$ & & $24 \pm 3.3$ & & $1.3 \pm 0.8$ \\
\hline Total Suspended Solids, mg/L & & 6 & . & 81 & & 8 & & 69 & & 45 \\
\hline Uranium, $\mu \mathrm{g} / \mathrm{g}$ & & 0.47 & & 0.054 & & 2.1 & & 0.007 & & $<0.002$ \\
\hline Chloromethane, $\mu \mathrm{g} / \mathrm{L}$ & & 10 & & 10 & & 10 & & 10 & & 10 \\
\hline Bromomethane, $\mu \mathrm{g} / \mathrm{L}$ & & 10 & & 10 & & 10 & & 10 & & 10 \\
\hline Vinyl chloride, $\mu \mathrm{g} / \mathrm{L}$ & & 10 & & 10 & & 10 & & 10 & & 10 \\
\hline Chloroethane, $\mu \mathrm{g} / \mathrm{L}$ & & 10 & & 10 & & 10 & & 10 & & 10 \\
\hline Trichlorofluoromethane, $\mu \mathrm{g} / \mathrm{L}$ & & 10 & & 10 & & 10 & & 10 & & 10 \\
\hline Methylene chloride, $\mu \mathrm{g} / \mathrm{L}$ & & 10 & & 10 & & 4 & & 7 & & 3 \\
\hline Acetone, $\mu \mathrm{g} / \mathrm{L}$ & & 2 & & 9 & & 8 & & 6 & & 5 \\
\hline
\end{tabular}


Table 4 (continued)

\begin{tabular}{|c|c|c|c|c|c|}
\hline Sample no. (location) & $\begin{array}{c}\text { BC1101 } \\
\text { (BC 1030) }\end{array}$ & $\begin{array}{c}\text { BC1102 } \\
\text { (BC 1280) }\end{array}$ & $\begin{array}{c}\text { NT1101 } \\
\text { (NT1 200) }\end{array}$ & $\begin{array}{c}\text { NT1102 } \\
\text { (NT1 390) }\end{array}$ & $\begin{array}{c}\text { NT2101 } \\
\text { (NT2 970) }\end{array}$ \\
\hline Collection date & $9 / 12 / 96$ & $9 / 20 / 96$ & $9 / 19 / 96$ & $9 / 19 / 96$ & $9 / 12 / 96$ \\
\hline
\end{tabular}

\begin{tabular}{|c|c|c|c|c|c|c|c|c|c|}
\hline & Filtered & Unfiltered & Filtered & Unfiltered & Filtered & Unfiltered & Filtered & Unfiltered Filtered & Unfiltered \\
\hline $\begin{array}{l}\text { 1,1,2-Trichloro- } \\
\text { 1,2,2-trifluoroethane, } \mu \mathrm{g} / \mathrm{L}\end{array}$ & & 10 & & 10 & & 3 & & 10 & 12 \\
\hline Carbon disulfide, $\mu \mathrm{g} / \mathrm{L}$ & & 10 & & 10 & & 10 & & 10 & 10 \\
\hline 1,1-Dichloroethene, $\mu \mathrm{g} / \mathrm{L}$ & & 10 & & 10 & & 10 & & 10 & 10 \\
\hline 1,1-Dichloroethane, $\mu \mathrm{g} / \mathrm{L}$ & & 10 & & 10 & & 10 & & 10 & 10 \\
\hline cis-1,2-Dichloroethene, $\mu \mathrm{g} / \mathrm{L}$ & & 10 & & 10 & & 2 & & 2 & 10 \\
\hline $\operatorname{tran}-1,2$-Dichloroethene, $\mu \mathrm{g} / \mathrm{L}$ & & 10 & & 10 & & 10 & & 10 & 10 \\
\hline Chloroform, $\mu \mathrm{g} / \mathrm{L}$ & & 10 & & 10 & & 1 & & 6 & 5 \\
\hline 1,2-Dichloroethane, $\mu \mathrm{g} / \mathrm{L}$ & & 10 & & 10 & & 10 & & 10 & 10 \\
\hline 2-Butanone, $\mu \mathrm{g} / \mathrm{L}$ & . & 4 & & 5 & & 10 & & 10 & 6 \\
\hline 1,1,1-Trichloroethane, $\mu \mathrm{g} / \mathrm{L}$ & & 10 & & 10 & & 10 & & 10 & 10 \\
\hline Carbon tetrachloride, $\mu \mathrm{g} / \mathrm{L}$ & & 10 & & 10 & & 10 & & 10 & 10 \\
\hline Bromodichloromethane, $\mu \mathrm{g} / \mathrm{L}$ & & 10 & & 10 & & 10 & & 10 & 10 \\
\hline 1,2-Dichloropropane, $\mu \mathrm{g} / \mathrm{L}$ & & 10 & & 10 & & 10 & & 10 & 10 \\
\hline $\begin{array}{l}\text { cis-1,3-Dichloropropene, } \\
\mu \mathrm{g} / \mathrm{L}\end{array}$ & & 10 & & 10 & & 10 & & 10 & 10 \\
\hline Trichloroethene, $\mu \mathrm{g} / \mathrm{L}$ & & 10 & & 10 & & 10 & & 10 & 10 \\
\hline Dibromochloromethane, $\mu \mathrm{g} / \mathrm{L}$ & & 10 & & 10 & & 10 & & 10 & 10 \\
\hline 1,1,2-Trichloroethane, $\mu \mathrm{g} / \mathrm{L}$ & & 10 & & 10 & & 10 & & 10 & 10 \\
\hline Benzene, $\mu \mathrm{g} / \mathrm{L}$ & & 10 & & 10 & & 10 & & 10 & 10 \\
\hline
\end{tabular}




\begin{tabular}{|c|c|c|c|c|c|c|c|c|c|c|}
\hline \multirow[t]{2}{*}{$\begin{array}{l}\text { Sample no. (location) } \\
\text { Collection date }\end{array}$} & \multicolumn{2}{|c|}{$\begin{array}{c}\text { BC1101 } \\
\text { (BC 1030) } \\
9 / 12 / 96\end{array}$} & \multicolumn{2}{|c|}{$\begin{array}{c}\text { BC1102 } \\
\text { (BC 1280) } \\
9 / 20 / 96\end{array}$} & \multicolumn{2}{|c|}{$\begin{array}{c}\text { NT1101 } \\
\text { (NT1 200) } \\
9 / 19 / 96\end{array}$} & \multicolumn{2}{|c|}{$\begin{array}{c}\text { NT1102 } \\
\text { (NT1 390) } \\
9 / 19 / 96\end{array}$} & \multicolumn{2}{|c|}{$\begin{array}{c}\text { NT2101 } \\
\text { (NT2 970) } \\
9 / 12 / 96\end{array}$} \\
\hline & Filtered & Unfiltered & Filtered & Unfiltered & Filtered & Unfiltered & Filtered & Unfiltered & Filtered & Unfiltered \\
\hline $\begin{array}{l}\text { trans-1,3-Dichloropropene, } \\
\mu \mathrm{g} / \mathrm{L}\end{array}$ & & 10 & & 10 & & 10 & & 10 & & 10 \\
\hline Bromoform, $\mu \mathrm{g} / \mathrm{L}$ & & 10 & & 10 & & 10 & & 10 & & 10 \\
\hline 4-Methyl-2-pentanone, $\mu \mathrm{g} / \mathrm{L}$ & & 10 & & 10 & & 10 & & 10 & & 10 \\
\hline 2-Hexanone, $\mu \mathrm{g} / \mathrm{L}$ & & 10 & & 10 & & 10 & & 10 & & 10 \\
\hline Tetrachloroethene, $\mu \mathrm{g} / \mathrm{L}$ & & 10 & & 10 & & 52 & & 45 & & 10 \\
\hline $\begin{array}{l}\text { 1,1,2,2-Tetrachloroethane, } \\
\mu \mathrm{g} / \mathrm{L}\end{array}$ & & 10 & & 10 & & 10 & & 10 & & 10 \\
\hline Toluene, $\mu \mathrm{g} / \mathrm{L}$ & & 10 & & 10 & & 10 & & 10 & & 10 \\
\hline Chlorobenzene, $\mu \mathrm{g} / \mathrm{L}$ & & 10 & & 10 & & 10 & & 10 & & 10 \\
\hline Ethylbenzene, $\mu \mathrm{g} / \mathrm{L}$ & & 10 & & 10 & & 10 & & 10 & & 10 \\
\hline Styrene, $\mu \mathrm{g} / \mathrm{L}$ & & 10 & & 10 & & 10 & & 10 & & 10 \\
\hline Xylene, $\mu \mathrm{g} / \mathrm{L}$ & & 10 & & 10 & & 10 & & 10 & & 10 \\
\hline
\end{tabular}




\section{4}

Table 5. Field measurements from seeps (see Table 4)

\begin{tabular}{cccccc}
\hline Location & Sample no. & Date & $\begin{array}{c}\text { Conductivity } \\
(\mathbf{m S} / \mathbf{c m})\end{array}$ & $\begin{array}{c}\text { pH } \\
(\mathbf{s u})\end{array}$ & $\begin{array}{c}\text { Temperature } \\
\left({ }^{\circ} \mathbf{C}\right)\end{array}$ \\
\hline BC 1030 & BC1101 & $9 / 12 / 96$ & 0.994 & 6.61 & 22.0 \\
BC 1280 & BC1102 & $9 / 20 / 96$ & 0.307 & 6.99 & 17.1 \\
NT1 200 & NT1101 & $9 / 19 / 96$ & 0.522 & 5.42 & 20.7 \\
NT1 390 & NT1102 & $9 / 19 / 96$ & 17.400 & 5.23 & 20.5 \\
NT2 970 & NT2101 & $9 / 12 / 96$ & 0.579 & 5.79 & 17.8 \\
\hline
\end{tabular}




\begin{tabular}{|c|c|c|c|c|c|c|c|c|c|}
\hline Location & $\begin{array}{c}\text { Surface } \\
\text { Elevation } \\
\text { (AMSL) }\end{array}$ & $\begin{array}{c}\text { Total depth } \\
\text { [from TOC (ft)] }\end{array}$ & $\begin{array}{l}\text { Casing } \\
\text { stick-up } \\
\text { (ft) }\end{array}$ & $\begin{array}{l}\text { TD (BLS) } \\
\text { (ft) }\end{array}$ & $\begin{array}{c}\text { Bedrock } \\
\text { Elevation } \\
\text { (AMSL) }\end{array}$ & $\begin{array}{c}\text { Depth to water } \\
\text { [from TOC } \\
\text { (ft)] } \\
6 / 24 / 96\end{array}$ & $\begin{array}{l}\text { Depth to water } \\
\text { [from BLS (ft)] } \\
6 / 24 / 96\end{array}$ & $\begin{array}{l}\text { Water Table } \\
\text { Elevation } \\
\text { (AMSL) }\end{array}$ & $\begin{array}{c}\text { Water } \\
\text { height } \\
(\mathrm{ft}) \\
6 / 24 / 96\end{array}$ \\
\hline TPB-1 & 990.33 & 16.51 & 3.57 & 12.94 & 977.39 & 12.16 & 8.59 & 981.74 & 4.35 \\
\hline TPB-2 & 991.72 & 20.88 & 3.08 & 17.8 & 973.92 & 9.50 & 6.42 & 985.30 & 11.38 \\
\hline TPB-3 & --- & Abandoned & - & -- & - & - & - & -- & $\cdots$ \\
\hline TPB-4 & 990.30 & 16.64 & 1.88 & 14.76 & 975.54 & 10.39 & 8.51 & 981.79 & 6.25 \\
\hline TPB-5 & 991.20 & 20.42 & 2.94 & 17.48 & 973.72 & 12.76 & 9.82 & 981.38 & 7.66 \\
\hline TPB-6 & 998.17 & 16.64 & 2.08 & 14.56 & 983.61 & 13.16 & 11.08 & 987.09 & 3.48 \\
\hline TPB-7 & 996.63 & 23.46 & 2.72 & 20.74 & 975.89 & 17.15 & 14.43 & 982.20 & 6.31 \\
\hline TPB-8 & 988.87 & 19.63 & 2.71 & 16.92 & 971.95 & 10.27 & 7.56 & 981.31 & 9.36 \\
\hline TPB-9 & 1004.51 & 16.63 & 2.35 & 14.28 & 990.23 & 10.94 & 8.59 & 995.92 & 5.69 \\
\hline TPB-10 & 1005.04 & 25.34 & 2.04 & 23.3 & 981.74 & 13.20 & 11.16 & 993.88 & 12.14 \\
\hline TPB-11 & 1003.08 & 20.42 & 2.49 & 17.93 & 985.15 & 13.68 & 11.19 & 991.89 & 6.74 \\
\hline TPB-12 & 975.63 & 16.02 & 3.12 & 12.9 & 962.73 & 8.83 & 5.71 & 969.92 & 7.19 \\
\hline TPB-13 & 969.94 & 19.99 & 2.63 & 17.36 & 952.58 & 5.82 & 3.19 & 966.75 & 14.17 \\
\hline TPB-14 & 971.70 & 11.68 & 1.96 & 9.72 & 961.98 & 8.98 & 7.02 & 964.68 & 2.70 \\
\hline TPB-15 & 997.91 & 19.85 & 2.32 & 17.53 & 980.38 & 13.18 & 10.86 & 987.05 & 6.67 \\
\hline TPB-16 & 998.21 & 16.58 & 1.29 & 15.29 & 982.92 & 12.30 & 11.01 & 987.20 & 4.28 \\
\hline TPB-17 & -- & Abandoned & $\cdots$ & -- & -- & - & - & -- & $-\cdots$ \\
\hline TPB-18 & 997.30 & 23.27 & 2.68 & 20.59 & 976.71 & 14.50 & 11.82 & 985.48 & 8.77 \\
\hline TPB-19 & 1001.36 & 13.97 & 2.79 & 11.18 & 990.18 & 8.68 & 5.89 & 995.47 & 5.29 \\
\hline TPB-20 & 975.75 & 14.84 & 2.52 & 12.32 & 963.43 & 9.36 & 6.84 & 968.91 & 5.48 \\
\hline
\end{tabular}


Table 6 (continued)

\begin{tabular}{|c|c|c|c|c|c|c|c|c|c|}
\hline Location & $\begin{array}{l}\text { Surface } \\
\text { Elevation } \\
\text { (AMSL) }\end{array}$ & $\begin{array}{c}\text { Total depth } \\
\text { [from TOC (ft)] }\end{array}$ & $\begin{array}{l}\text { Casing } \\
\text { stick-up } \\
\text { (ft) }\end{array}$ & $\begin{array}{l}\text { TD (BLS) } \\
\text { (ft) }\end{array}$ & $\begin{array}{c}\text { Bedrock } \\
\text { Elevation } \\
\text { (AMSL) }\end{array}$ & $\begin{array}{c}\text { Depth to water } \\
\text { [from TOC } \\
\text { (ft)] } \\
6 / 24 / 96 \\
\end{array}$ & $\begin{array}{l}\text { Depth to water } \\
\text { [from BLS (ft)] } \\
6 / 24 / 96 \\
\end{array}$ & $\begin{array}{l}\text { Water Table } \\
\text { Elevation } \\
\text { (AMSL) }\end{array}$ & $\begin{array}{c}\text { Water } \\
\text { height } \\
\text { (ft) } \\
6 / 24 / 96\end{array}$ \\
\hline TPB-21 & 980.97 & 19.08 & 2.42 & 16.66 & 964.31 & 17.83 & 15.41 & 965.56 & 1.25 \\
\hline TPB-22 & 980.13 & 21.53 & 2.29 & 19.24 & 960.89 & 17.35 & 15.06 & 965.07 & 4.18 \\
\hline TPB-23 & 972.76 & 23.81 & 2.68 & 21.13 & 951.63 & 10.62 & 7.94 & 964.82 & 13.19 \\
\hline TPB-24 & 991.53 & 18.65 & 2.67 & 15.98 & 975.55 & 11.95 & 9.28 & 982.25 & 6.70 \\
\hline TPB-25 & 1007.68 & 16.56 & 1.41 & $15: 15$ & 992.53 & 12.15 & 10.74 & 996.94 & 4.41 \\
\hline TPB-26 & 1000.36 & 19.85 & 2.79 & 17.06 & 983.30 & 9.82 & 7.03 & 993.33 & 10.03 \\
\hline TPB-27 & 998.72 & 21.53 & 2.68 & 18.85 & 979.87 & 11.17 & 8.49 & 990.23 & 10.36 \\
\hline TPB-28 & 1010.69 & 23.10 & 3.00 & 20.10 & 990.59 & $15.64^{a}$ & $12.64^{a}$ & 998.05 & $7.46^{a}$ \\
\hline TPB-29 & 999.88 & 23.10 & 3.00 & 20.10 & 979.78 & $14.86^{a}$ & $11.86^{a}$ & 988.02 & $8.24^{a}$ \\
\hline TPB-30 & 1000.06 & 22.60 & 3.50 & 18.60 & 981.46 & $13.08^{a}$ & $9.58^{a}$ & 990.48 & $9.52^{a}$ \\
\hline TPB-31 & 1007.42 & 13.30 & 3.40 & 9.90 & $b$ & $11.68^{a}$ & $8.28^{a}$ & 999.14 & $1.62^{a}$ \\
\hline TPB-32 & 1007.29 & 19.30 & 3.20 & 16.10 & 991.19 & $14.46^{a}$ & $11.26^{a}$ & 996.03 & $4.84^{a}$ \\
\hline
\end{tabular}

- Measurements made 11/6/96. These piezometers were installed in a later phase.

bush probe refusal encountered above bedrock. 
Table 7. Augered piezometer boring information

\begin{tabular}{|c|c|c|c|c|c|c|c|c|c|}
\hline Location & $\begin{array}{c}\text { Surface } \\
\text { Elevation }\end{array}$ & $\begin{array}{l}\text { Total depth } \\
\text { [from TOC } \\
\text { (ft)] }\end{array}$ & $\begin{array}{c}\text { Casing stick-up } \\
\text { (ft) }\end{array}$ & $\begin{array}{l}\text { TD (BLS) } \\
\text { (ft) }\end{array}$ & $\begin{array}{l}\text { Bedrock } \\
\text { Elevation } \\
\text { (AMSI) }\end{array}$ & $\begin{array}{c}\text { Depth to Water } \\
\text { [from TOC } \\
\text { (ft)] }\end{array}$ & $\begin{array}{l}\text { Depth to water } \\
\text { [from BLS (ft)] }\end{array}$ & $\begin{array}{c}\text { Water } \\
\text { Table } \\
\text { Elevation } \\
\text { (AMSL) }\end{array}$ & $\begin{array}{c}\text { Water } \\
\text { height } \\
\text { (ft) }\end{array}$ \\
\hline GW-834 & 992.05 & 19.80 & 3.00 & 16.80 & 975.25 & $13.90(8 / 20 / 96)$ & 10.90 & 981.15 & 5.90 \\
\hline GW-835 & 998.04 & 22.10 & 2.90 & 19.2 & 978.84 & $13.23(8 / 14 / 96)$ & 10.33 & 987.71 & 8.87 \\
\hline GW-836 & 998.04 & 27.35 & 3.00 & 24.35 & 973.69 & $16.12(8 / 28 / 96)$ & 13.12 & 984.92 & 11.23 \\
\hline GW-837 & 1005.22 & 30.90 & 3.00 & 27.90 & 977.32 & $12.94(8 / 19 / 96)$ & 9.94 & 995.28 & 17.96 \\
\hline
\end{tabular}




\begin{tabular}{|c|c|c|c|c|c|c|c|c|c|c|}
\hline \multirow{4}{*}{$\begin{array}{l}\text { Sample No. (location) } \\
\text { Collection date } \\
\text { Analysis }\end{array}$} & \multicolumn{4}{|c|}{ PA1001 (GW-834) } & \multicolumn{2}{|c|}{ PA1002 (GW-835) } & \multicolumn{4}{|c|}{ PA1004 (GW-837) } \\
\hline & \multicolumn{4}{|c|}{$8 / 20 / 96$} & \multicolumn{2}{|c|}{$8 / 14 / 96$} & \multicolumn{4}{|c|}{$8 / 19 / 96$} \\
\hline & \multicolumn{2}{|c|}{ Filtered } & \multicolumn{2}{|c|}{ Unfiltered } & \multirow{2}{*}{$\frac{\text { Filtered }}{\text { Rep 1 }}$} & \multirow{2}{*}{$\frac{\text { Unfiltered }}{\text { Rep 2 }}$} & \multicolumn{2}{|c|}{ Filtered } & \multicolumn{2}{|c|}{ Unfiltered } \\
\hline & Rep 1 & Rep 2 & Rep 1 & $\operatorname{Rep} 2$ & & & Rep 1 & $\operatorname{Rep} 2$ & $\operatorname{Rep} 1$ & $\operatorname{Rep} 2$ \\
\hline Bicarbonate, $\mathrm{mg} / \mathrm{L}$ & & & 443 & & & 426 & & & 434 & \\
\hline Mercury, mg/L & 0.0002 & & $<0.0002$ & & & 0.00049 & 0.00039 & & 0.0012 & \\
\hline Aluminum, $\mu \mathrm{g} / \mathrm{L}$ & 2760 & 2670 & 9860 & 8210 & 1220 & 4550 & 4690 & 4630 & 27900 & 25500 \\
\hline Boron, $\mu \mathrm{g} / \mathrm{L}$ & 102 & $<100$ & 114 & $<100$ & 108 & 108 & 23.1 & $<100$ & 39.9 & $<100$ \\
\hline Barium, $\mu \mathrm{g} / \mathrm{L}$ & $>23200$ & 23800 & 23700 & 24300 & 83.5 & 95.2 & 483 & 489 & 578 & 587 \\
\hline Calcium, $\mu \mathrm{g} / \mathrm{L}$ & $>1000000$ & 2629000 & $>1000000$ & 2662000 & 163000 & 163000 & $>1000000$ & 1647000 & $>1000000$ & 1681000 \\
\hline Cadmium, $\mu \mathrm{g} / \mathrm{L}$ & 411 & 391 & 427 & 404 & $<20$ & $<20$ & 199 & 184 & 204 & 196 \\
\hline Cobalt, $\mu \mathrm{g} / \mathrm{L}$ & 216 & 212 & 222 & 211 & $<20$ & $<20$ & 257 & 250 & 265 & 265 \\
\hline Chromium, $\mu \mathrm{g} / \mathrm{L}$ & $<20$ & $<100$ & $<20$ & $<100$ & $<20$ & $<20$ & $<20$ & $<100$ & $<20$ & $<100$ \\
\hline Copper, $\mu \mathrm{g} / \mathrm{L}$ & 65.2 & $<200$ & 70.5 & $<200$ & $<30$ & $<30$ & 58.6 & $<200$ & 77.2 & $<200$ \\
\hline Iron, $\mu \mathrm{g} / \mathrm{L}$ & 362 & $<800$ & 5810 & 5700 & 712 & 4580 & 207 & $<800$ & 14300 & 14300 \\
\hline Potassium, $\mu \mathrm{g} / \mathrm{L}$ & 29000 & 26500 & 32000 & 28200 & 6380 & 7300 & 43200 & 38800 & 47900 & 42400 \\
\hline Magnesium, $\mu \mathrm{g} / \mathrm{L}$ & 335000 & 322000 & 343000 & 327000 & 26000 & 26400 & 152000 & 146000 & 158000 & 151000 \\
\hline Manganese, $\mu \mathrm{g} / \mathrm{L}$ & $>100000$ & 145000 & $>100000$ & 147000 & 1120 & 1160 & 100000 & 157000 & $>100000$ & 159000 \\
\hline Molybdenum, $\mu \mathrm{g} / \mathrm{L}$ & $<40$ & $<200$ & $<40$ & $<200$ & $<40$ & $<40$ & $<40$ & $<200$ & $<40$ & $<200$ \\
\hline Sodium, $\mu \mathrm{g} / \mathrm{L}$ & 506000 & 494000 & 516000 & 500000 & 17400 & 17300 & 695000 & 677000 & 705000 & 688000 \\
\hline Nickel, $\mu \mathrm{g} / \mathrm{L}$ & 1660 & 1710 & 1710 & 1750 & $<40$ & $<40$ & 2130 & 2130 & 2140 & 2210 \\
\hline Lead, $\mu \mathrm{g} / \mathrm{L}$ & $<200$ & $<800$ & $<200$ & $<800$ & $<200$ & $<200$ & $<200$ & $<800$ & $<200$ & $<800$ \\
\hline Thallium, $\mu \mathrm{g} / \mathrm{L}$ & 136 & $<500$ & 139 & $<500$ & $<100$ & $<100$ & 143 & $<500$ & 169 & $<500$ \\
\hline Vanadium, $\mu \mathrm{g} / \mathrm{L}$ & $<10$ & $<50$ & $<10$ & $<50$ & $<10$ & $<10$ & $<10$ & $<50$ & $<10$ & $<50$ \\
\hline
\end{tabular}




\begin{tabular}{|c|c|c|c|c|c|c|c|c|c|c|}
\hline \multicolumn{11}{|c|}{ Table 8 (continued) } \\
\hline \multirow{4}{*}{$\begin{array}{l}\text { Sample No. (location) } \\
\text { Collection date } \\
\text { Analysis }\end{array}$} & \multicolumn{4}{|c|}{ PA1001 (GW-834) } & \multicolumn{2}{|c|}{ PA1002 (GW-835) } & \multicolumn{4}{|c|}{ PA1004 (GW-837) } \\
\hline & \multicolumn{4}{|c|}{$8 / 20 / 96$} & \multicolumn{2}{|c|}{$8 / 14 / 96$} & \multicolumn{4}{|c|}{$8 / 19 / 96$} \\
\hline & \multicolumn{2}{|c|}{ Filtered } & \multicolumn{2}{|c|}{ Unfiltered } & \multirow{2}{*}{$\frac{\text { Filtered }}{\text { Rep 1 }}$} & \multirow{2}{*}{$\frac{\text { Unfiltered }}{\text { Rep 2 }}$} & \multicolumn{2}{|c|}{ Filtered } & \multicolumn{2}{|c|}{ Unfiltered } \\
\hline & Rep 1 & $\operatorname{Rep} 2$ & Rep 1 & Rep 2 & & & Rep 1 & $\operatorname{Rep} 2$ & Rep 1 & Rep 2 \\
\hline Zinc, $\mu \mathrm{g} / \mathrm{L}$ & 33.5 & $<100$ & 44.8 & $<100$ & $<20$ & $<20$ & 97.6 & $<100$ & 119 & 105 \\
\hline Selenium, $\mu \mathrm{g} / \mathrm{L}$ & $<500$ & $<3000$ & $<500$ & $<3000$ & $<500$ & $<500$ & $<500$ & $<3000$ & $<500$ & $<3000$ \\
\hline Silver, $\mu \mathrm{g} / \mathrm{L}$ & $<20$ & $<80$ & $<20$ & $<80$ & $<20$ & $<20$ & $<20$ & $<80$ & $<20$ & $<80$ \\
\hline Arsenic, $\mu \mathrm{g} / \mathrm{L}$ & $<100$ & $<500$ & $<100$ & $<500$ & $<100$ & $<100$ & $<100$ & $<500$ & $<100$ & $<500$ \\
\hline Beryllium, $\mu \mathrm{g} / \mathrm{L}$ & 2 & $<5.0$ & 2.5 & $<5.0$ & $<1.0$ & $<1.0$ & 2.9 & $<5.0$ & 4.5 & $<5$ \\
\hline Antimony, $\mu \mathrm{g} / \mathrm{L}$ & $<100$ & $<500$ & 100 & $<500$ & $<100$ & $<100$ & $<100$ & $<500$ & $<100$ & $<500$ \\
\hline Lithium, $\mu \mathrm{g} / \mathrm{L}$ & 41.8 & $<50$ & 50.5 & $<50$ & 30.6 & 32.4 & 119 & 93.8 & 143 & 124 \\
\hline Phosphorous, $\mu \mathrm{g} / \mathrm{L}$ & 547 & $<2000$ & 668 & $<2000$ & $<300$ & $<300$ & 562 & $<2000$ & 724 & $<2000$ \\
\hline Titanium, $\mu \mathrm{g} / \mathrm{L}$ & $<20$ & $<80$ & 189 & 96.2 & $<20$ & 124 & $<20$ & $<80$ & 244 & 161 \\
\hline Strontium, $\mu \mathrm{g} / \mathrm{L}$ & 660 & 6770 & 6670 & 6850 & 488 & 490 & 2390 & 2440 & 2450 & 2500 \\
\hline Alkalinity, $\mathrm{mg} / \mathrm{L}$ & & & 420 & & & 310.1 & & & 410 & \\
\hline Alpha activity, $\mathrm{pC} / \mathrm{L}$ & & & $210 \pm 56$ & & & $370 \pm 45$ & & & $790 \pm 120$ & \\
\hline Beta activity, $\mathrm{pC} / \mathrm{L}$ & & & $9000 \pm 1000$ & & & $280 \pm 34$ & & & $4300 \pm 500$ & \\
\hline Tritium, $\mathrm{pC} / \mathrm{L}$ & & & $1500 \pm 280$ & & & $-46 \pm 150$ & & & $1100 \pm 250$ & \\
\hline Fluoride, $\mathrm{mg} / \mathrm{L}$ & & & 4.8 & & & 0.4 & & & 4.9 & \\
\hline Chloride, $\mathrm{mg} / \mathrm{L}$ & & & 260 & & & 28 & & & 320 & \\
\hline Nitrite as Nitrogen, $\mathrm{mg} / \mathrm{L}$ & & & 8.3 & & & 0.2 & & & 7.3 & \\
\hline Bromide, $\mathrm{mg} / \mathrm{L}$ & & & NA & & & NA & & & NA & \\
\hline Nitrate as Nitrogen, $\mathrm{mg} / \mathrm{L}$ & & & 2300 & & & 29 & & & 1400 & \\
\hline
\end{tabular}




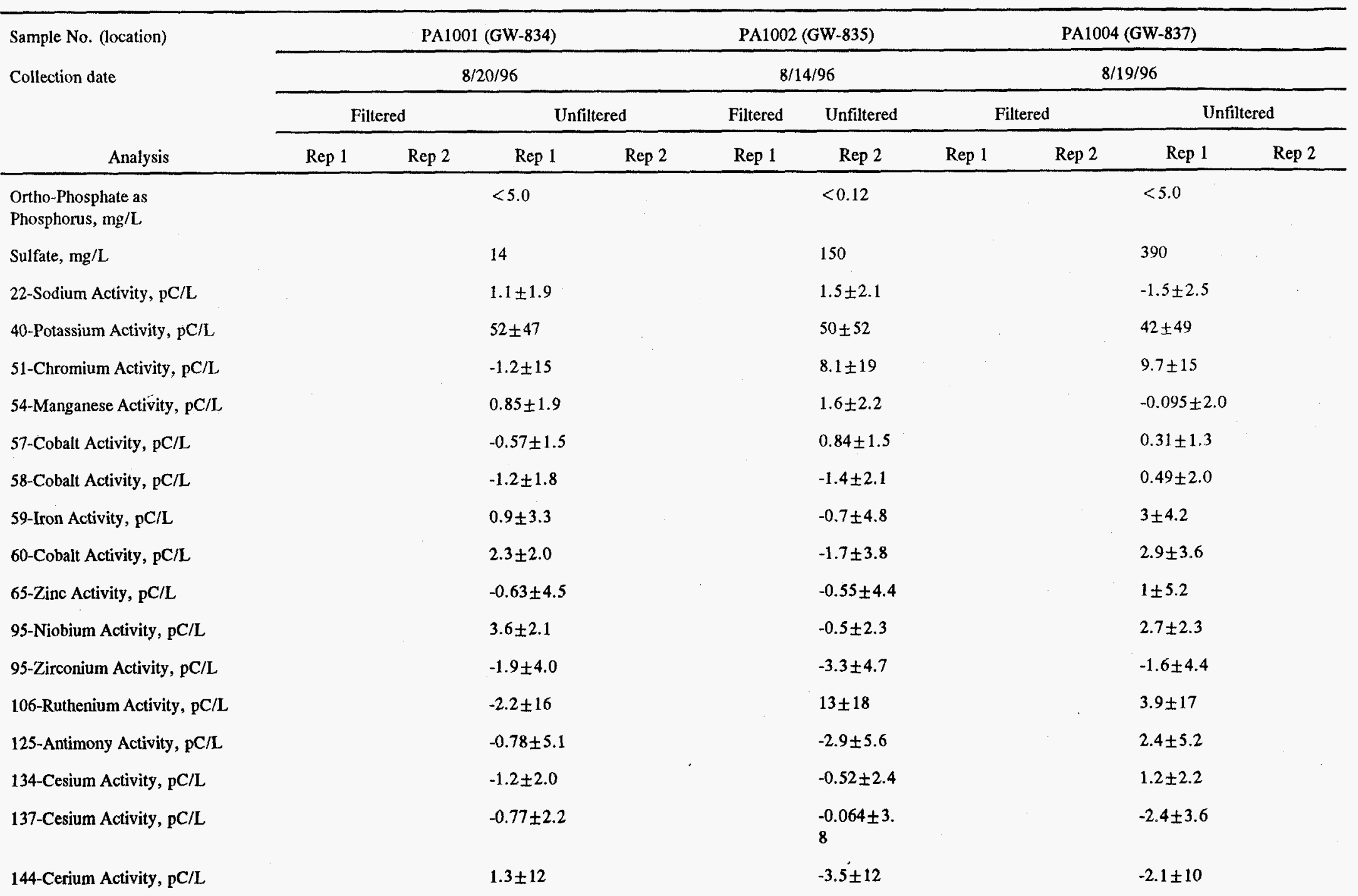




\begin{tabular}{|c|c|c|c|c|c|c|c|c|c|c|c|}
\hline \multirow{4}{*}{$\begin{array}{l}\text { Sample No. (location) } \\
\text { Collection date } \\
\text { Analysis }\end{array}$} & \multicolumn{4}{|c|}{ PA1001 (GW-834) } & \multicolumn{2}{|c|}{ PA1002 (GW-835) } & \multicolumn{4}{|c|}{ PA1004 (GW-837) } & \multirow{4}{*}{ * } \\
\hline & \multicolumn{4}{|c|}{$8 / 20 / 96$} & \multicolumn{2}{|c|}{$8 / 14 / 96$} & \multicolumn{4}{|c|}{$8 / 19 / 96$} & \\
\hline & \multicolumn{2}{|c|}{ Filtered } & \multicolumn{2}{|c|}{ Unfiltered } & \multirow{2}{*}{$\frac{\text { Filtered }}{\text { Rep } 1}$} & \multirow{2}{*}{$\frac{\text { Unfiltered }}{\text { Rep } 2}$} & \multicolumn{2}{|c|}{ Filtered } & \multicolumn{2}{|c|}{ Unfiltered } & \\
\hline & Rep 1 & Rep 2 & Rep 1 & Rep 2 & & & Rep 1 & Rep 2 & Rep 1 & Rep 2 & \\
\hline 208-Thallium Activity, $\mathrm{pC} / \mathrm{L}$ & & & $4.5 \pm 4.4$ & & & $3.4 \pm 4.0$ & & & $3.1 \pm 3.6$ & & \\
\hline 212-Bismuth Activity, $\mathrm{pC} / \mathrm{L}$ & & & $13 \pm 14$ & & & $13 \pm 16$ & & & $1.8 \pm 16$ & & \\
\hline 212-Lead Activity, $\mathrm{pC} / \mathrm{L}$ & & & $18 \pm 5.6$ & & & $6.4 \pm 5.7$ & & & $12 \pm 5.0$ & & \\
\hline 235 Uranium Activity, $\mathrm{pC} / \mathrm{L}$ & & & $7.8 \pm 5.0$ & & & $5 \pm 18$ & & & $20 \pm 5.0$ & & \\
\hline Technetium -99, pC/L & & & $\begin{array}{l}22000 \\
\pm 2200\end{array}$ & & & $150 \pm 16$ & & & $\begin{array}{l}10000 \\
\pm 1000\end{array}$ & & \\
\hline Total Dissolved Solids, mg/L & & & & & & 690 & & & 11000 & & \\
\hline Total Strontium, $\mathrm{pC} / \mathrm{L}$ & & & $-2.4 \pm ?$ & & & $0.4 \pm 1.1$ & & & $360 \pm ?$ & & $\omega$ \\
\hline Total Suspended Solids, mg/L & & & 190 & & & 9 & & & & & \\
\hline Uranium, $\mu \mathrm{g} / \mathrm{g}$ & & & 0.0037 & & & 1.7 & & & 2.6 & & \\
\hline Unknown, $\mu \mathrm{g} / \mathrm{L}$ & & & 65 & & & & & & 60 & & \\
\hline Acetaldoxime, $\mu \mathrm{g} / \mathrm{L}$ & & & 6 & & & & & & & & \\
\hline 1,2-Ethanediol, Dinitrate, $\mu \mathrm{g} / \mathrm{L}$ & & & 16 & & & & & & & & \\
\hline 1,5-Pentanediol, Dinitrate, $\mu \mathrm{g} / \mathrm{L}$ & & & 10 & & & & & & & & \\
\hline Chloromethane, $\mu \mathrm{g} / \mathrm{L}$ & & & $10 \mathrm{U}$ & & & $10 U$ & & & $3 \mathrm{~J}$ & & \\
\hline Bromomethane, $\mu \mathrm{g} / \mathrm{L}$ & & & $4 \mathrm{~J}$ & & & $10 U$ & & & $2 \mathrm{~J}$ & & \\
\hline Vinyl chloride, $\mu \mathrm{g} / \mathrm{L}$ & & & $10 \mathrm{U}$ & & & $10 \mathrm{U}$ & & & $10 U$ & & \\
\hline Chloroethane, $\mu \mathrm{g} / \mathrm{L}$ & & & $10 U$ & & & $10 \mathrm{U}$ & & & $10 \mathrm{U}$ & & \\
\hline Trichlorofluoromethane, $\mu \mathrm{g} / \mathrm{L}$ & & & $10 \mathrm{U}$ & & & $10 \mathrm{U}$ & & & $10 \mathrm{U}$ & & \\
\hline Methylene chloride, $\mu \mathrm{g} / \mathrm{L}$ & & & 14 & & & $10 \mathrm{U}$ & & & 18 & & \\
\hline
\end{tabular}




\begin{tabular}{|c|c|c|c|c|c|c|c|c|c|c|}
\hline \multicolumn{11}{|c|}{ Table 8 (continued) } \\
\hline \multirow{4}{*}{$\begin{array}{l}\text { Sample No. (location) } \\
\text { Collection date } \\
\text { Analysis }\end{array}$} & \multicolumn{4}{|c|}{ PA1001 (GW-834) } & \multicolumn{2}{|c|}{ PA1002 (GW-835) } & \multicolumn{4}{|c|}{ PA1004 (GW-837) } \\
\hline & \multicolumn{4}{|c|}{$8 / 20 / 96$} & \multicolumn{2}{|c|}{$8 / 14 / 96$} & \multicolumn{4}{|c|}{$8 / 19 / 96$} \\
\hline & \multicolumn{2}{|c|}{ Filtered } & \multicolumn{2}{|c|}{ Unfiltered } & \multirow{2}{*}{$\begin{array}{l}\text { Filtered } \\
\text { Rep } 1 \\
\end{array}$} & \multirow{2}{*}{$\begin{array}{c}\text { Unfiltered } \\
\text { Rep } 2 \\
\end{array}$} & \multicolumn{2}{|c|}{ Filtered } & \multicolumn{2}{|c|}{ Unfiltered } \\
\hline & Rep 1 & Rep 2 & Rep 1 & Rep 2 & & & Rep 1 & Rep 2 & Rep 1 & $\operatorname{Rep} 2$ \\
\hline Acetone, $\mu \mathrm{g} / \mathrm{L}$ & \multicolumn{4}{|c|}{12} & \multicolumn{2}{|r|}{$9 \mathrm{BJ}$} & \multicolumn{4}{|c|}{43} \\
\hline $\begin{array}{l}\text { 1,1,2-Trichloro- } \\
\text { 1,2,2-trifluoroethane, } \mu \mathrm{g} / \mathrm{L}\end{array}$ & \multicolumn{4}{|c|}{$10 \mathrm{U}$} & \multicolumn{2}{|r|}{$5 \mathrm{~J}$} & \multicolumn{4}{|c|}{$3 \mathrm{~J}$} \\
\hline Carbon disulfide, $\mu \mathrm{g} / \mathrm{L}$ & \multicolumn{4}{|c|}{$10 \mathrm{U}$} & \multicolumn{2}{|r|}{$10 U$} & \multicolumn{4}{|c|}{$10 \mathrm{U}$} \\
\hline 1,1-Dichloroethene, $\mu \mathrm{g} / \mathrm{L}$ & \multicolumn{4}{|c|}{$10 \mathrm{U}$} & \multicolumn{2}{|r|}{$10 \mathrm{U}$} & \multicolumn{4}{|c|}{$10 \mathrm{U}$} \\
\hline 1,1-Dichloroethane, $\mu \mathrm{g} / \mathrm{L}$ & \multicolumn{4}{|c|}{$10 \mathrm{U}$} & \multicolumn{2}{|r|}{$10 \mathrm{U}$} & \multicolumn{4}{|c|}{$10 \mathrm{U}$} \\
\hline cis-1,2-Dichloroethene, $\mu \mathrm{g} / \mathrm{L}$ & \multicolumn{4}{|c|}{$6 \mathrm{~J}$} & \multicolumn{2}{|r|}{$10 \mathrm{U}$} & \multicolumn{4}{|c|}{$3 \mathrm{~J}$} \\
\hline tran-1,2-Dichloroethene, $\mu \mathrm{g} / \mathrm{L}$ & & & $10 \mathrm{U}$ & & & $10 \mathrm{U}$ & & & $10 \mathrm{U}$ & \\
\hline Chloroform, $\mu \mathrm{g} / \mathrm{L}$ & & & $9 \mathrm{~J}$ & & & $10 \mathrm{U}$ & & & $7 \mathrm{~J}$ & \\
\hline 1,2-Dichloroethane, $\mu \mathrm{g} / \mathrm{L}$ & & & $10 \mathrm{U}$ & & & $10 \mathrm{U}$ & & & $10 \mathrm{U}$ & \\
\hline 2-Butanone, $\mu \mathrm{g} / \mathrm{L}$ & & & 4 BJ & & & $3 \mathrm{BJ}$ & & & $8 \mathrm{BJ}$ & \\
\hline 1,1,1-Trichloroethane, $\mu \mathrm{g} / \mathrm{L}$ & & & $10 \mathrm{U}$ & & & $10 \mathrm{U}$ & & & $1 \mathrm{~J}$ & \\
\hline Carbon tetrachioride, $\mu \mathrm{g} / \mathrm{L}$ & & & $10 \mathrm{U}$ & & & $10 U$ & & & $10 \mathrm{U}$ & \\
\hline Bromodichloromethane, $\mu \mathrm{g} / \mathrm{L}$ & & & $10 \mathrm{U}$ & & & $10 \mathrm{U}$ & & & $10 \mathrm{U}$ & \\
\hline 1,2-Dichloropropane, $\mu \mathrm{g} / \mathrm{L}$ & & & $10 \mathrm{U}$ & & & $10 \mathrm{U}$ & & & $10 \mathrm{U}$ & \\
\hline cis-1,3-Dichloropropene, $\mu \mathrm{g} / \mathrm{L}$ & & & $10 \mathrm{U}$ & & & $10 \mathrm{U}$ & & & $10 \mathrm{U}$ & \\
\hline Trichloroethene, $\mu \mathrm{g} / \mathrm{L}$ & & & $10 \mathrm{U}$ & & & $10 U$ & & & $10 \mathrm{U}$ & \\
\hline Dibromochloromethane, $\mu \mathrm{g} / \mathrm{L}$ & & & $10 \mathrm{U}$ & & & $10 \mathrm{U}$ & & & $10 \mathrm{U}$ & \\
\hline 1,1,2-Trichloroethane, $\mu \mathrm{g} / \mathrm{L}$ & & & $10 \mathrm{U}$ & & & $10 U$ & & & $10 \mathrm{U}$ & \\
\hline Benzene, $\mu \mathrm{g} / \mathrm{L}$ & & & $10 \mathrm{U}$ & & & $10 \mathrm{U}$ & & & $10 \mathrm{U}$ & \\
\hline
\end{tabular}




\begin{tabular}{|c|c|c|c|c|c|c|c|c|c|c|}
\hline \multirow{4}{*}{$\begin{array}{l}\text { Sample No. (location) } \\
\text { Collection date } \\
\text { Analysis }\end{array}$} & \multicolumn{4}{|c|}{ PA1001 (GW-834) } & \multicolumn{2}{|c|}{ PA1002 (GW-835) } & \multicolumn{4}{|c|}{ PA1004 (GW-837) } \\
\hline & \multicolumn{4}{|c|}{$8 / 20 / 96$} & \multicolumn{2}{|c|}{$8 / 14 / 96$} & \multicolumn{4}{|c|}{$8 / 19 / 96$} \\
\hline & \multicolumn{2}{|c|}{ Filtered } & \multicolumn{2}{|c|}{ Unfiltered } & \multirow{2}{*}{$\frac{\text { Filtered }}{\text { Rep } 1}$} & \multirow{2}{*}{$\begin{array}{c}\text { Unfiltered } \\
\text { Rep } 2\end{array}$} & \multicolumn{2}{|c|}{ Filtered } & \multicolumn{2}{|c|}{ Unfiltered } \\
\hline & $\operatorname{Rep} 1$ & Rep 2 & Rep 1 & $\operatorname{Rep} 2$ & & & Rep 1 & $\operatorname{Rep} 2$ & Rep 1 & Rep 2 \\
\hline trans-1,3-Dichloropropene, $\mu \mathrm{g} / \mathrm{L}$ & & & $10 \mathrm{U}$ & & & $10 \mathrm{U}$ & & & $10 \mathrm{U}$ & \\
\hline Bromoform, $\mu \mathrm{g} / \mathrm{L}$ & & & $10 \mathrm{U}$ & & & $10 \mathrm{U}$ & & & $10 \mathrm{U}$ & \\
\hline 4-Methyl-2-pentanone, $\mu \mathrm{g} / \mathrm{L}$ & & & $10 \mathrm{U}$ & & & $10 \mathrm{U}$ & & & $2 \mathrm{~J}$ & \\
\hline 2-Hexanone, $\mu \mathrm{g} / \mathrm{L}$ & & & $10 \mathrm{U}$ & & & $10 \mathrm{U}$ & & & $10 \mathrm{U}$ & \\
\hline Tetrachloroethene, $\mu \mathrm{g} / \mathrm{L}$ & & & $2 \mathrm{~J}$ & & & $4 \mathrm{~J}$ & & & 14 & \\
\hline 1,1,2,2-Tetrachloroethane, $\mu \mathrm{g} / \mathrm{L}$ & & & $10 \mathrm{U}$ & & & $10 \mathrm{U}$ & & & $10 \mathrm{U}$ & \\
\hline Toluene, $\mu \mathrm{g} / \mathrm{L}$ & & & $10 \mathrm{U}$ & & & $10 \mathrm{U}$ & & & $10 \mathrm{U}$ & \\
\hline Chlorobenzene, $\mu \mathrm{g} / \mathrm{L}$ & & & $10 \mathrm{U}$ & & & $10 \mathrm{U}$ & & & $10 \mathrm{U}$ & \\
\hline Ethylbenzene, $\mu \mathrm{g} / \mathrm{L}$ & & & $10 \mathrm{U}$ & & & $10 \mathrm{U}$ & & & $10 \mathrm{U}$ & \\
\hline Styrene, $\mu \mathrm{g} / \mathrm{L}$ & & & $10 \mathrm{U}$ & & & $10 \mathrm{U}$ & & & $10 \mathrm{U}$ & \\
\hline Xylene, $\mu \mathrm{g} / \mathrm{L}$ & & & $10 \mathrm{U}$ & & & $10 \mathrm{U}$ & & & $10 \mathrm{U}$ & \\
\hline Ethane 1,2-Dichloro-1,1,2-tr, $\mu \mathrm{g} / \mathrm{L}$ & & & & & & 4.03 & & & & \\
\hline
\end{tabular}


34

Table 9. Groundwater field measurements (see Table 8)

\begin{tabular}{cccccc}
\hline Location & Sample no. & Date & $\begin{array}{c}\text { Conductivity } \\
(\mathbf{m S} / \mathbf{c m})\end{array}$ & $\begin{array}{c}\text { pH } \\
(\mathbf{s u})\end{array}$ & $\begin{array}{c}\text { Temperature } \\
\left({ }^{\circ} \mathbf{C}\right)\end{array}$ \\
\hline GW-834 & PA1001 & $8 / 20 / 96$ & 16.5 & 5.48 & 23.8 \\
GW-835 & PA1002 & $8 / 14 / 96$ & 1.12 & 6.41 & 22.6 \\
GW-837 & PA1004 & $8 / 19 / 96$ & 11.9 & 5.38 & 26.4 \\
\hline
\end{tabular}


Table 10. Migration pathway summary chemical characteristics

\begin{tabular}{ccccccc}
\hline $\begin{array}{c}\text { Migration } \\
\text { pathway }\end{array}$ & $\begin{array}{c}\text { Sample } \\
\text { location }\end{array}$ & $\begin{array}{c}\text { Nitrate } \\
(\mathrm{mg} / \mathrm{L})\end{array}$ & $\begin{array}{c}\text { Uranium } \\
(\mu \mathrm{g} / \mathrm{g})\end{array}$ & $\begin{array}{c}\text { Gross alpha } \\
(\mathrm{pCi} / \mathrm{L})\end{array}$ & $\begin{array}{c}\text { Gross beta } \\
(\mathrm{pCi} / \mathrm{L})\end{array}$ & $\begin{array}{c}\text { Technetium } \\
(\mathbf{p C i} / \mathrm{L})\end{array}$ \\
\hline 1 & GW-837 & 1400 & 2.6 & 790 & 4300 & 10,000 \\
2 & GW-835 & 29 & 1.7 & 370 & 280 & 150 \\
3 & GW-834 & 2300 & 0.0037 & 210 & 9000 & 22,000 \\
3 (cont.) & TPB-12, -13, -20 & $2->50$ & $<0.003-0.037$ & $97-1581$ & $78-824$ & N/A \\
\hline
\end{tabular}



FIGURES 
' 


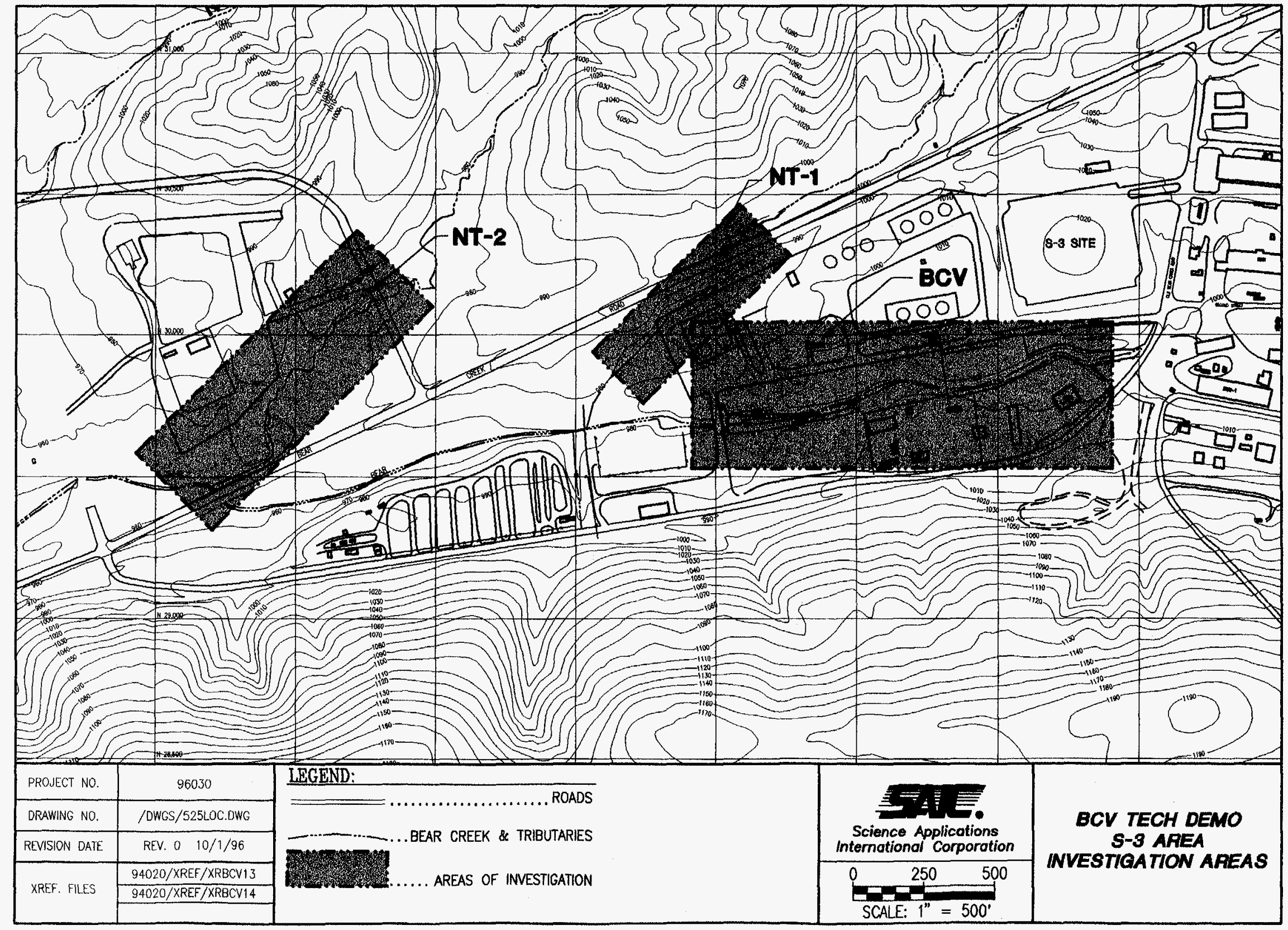

Fig. 1. Areas of investigation. 



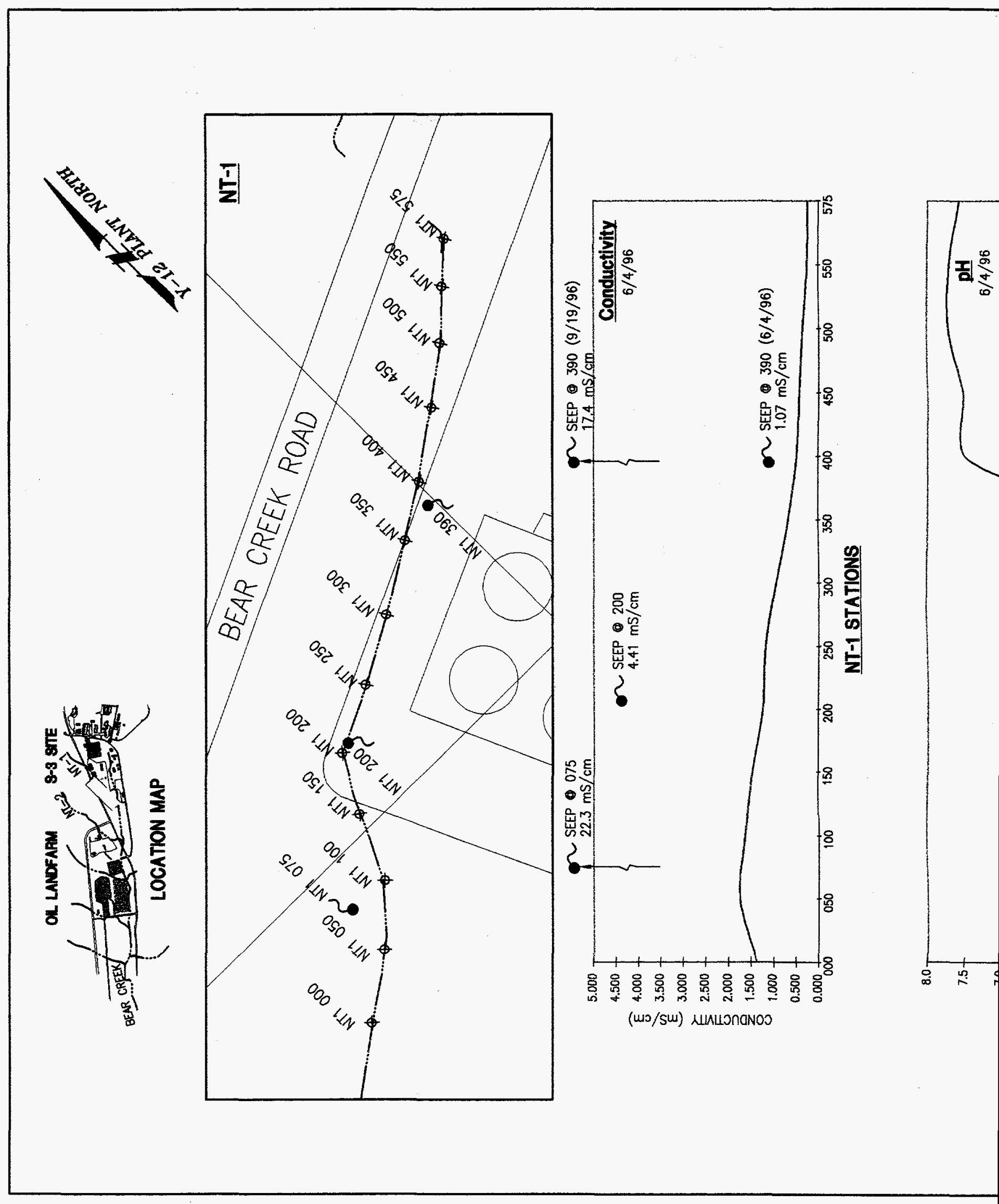




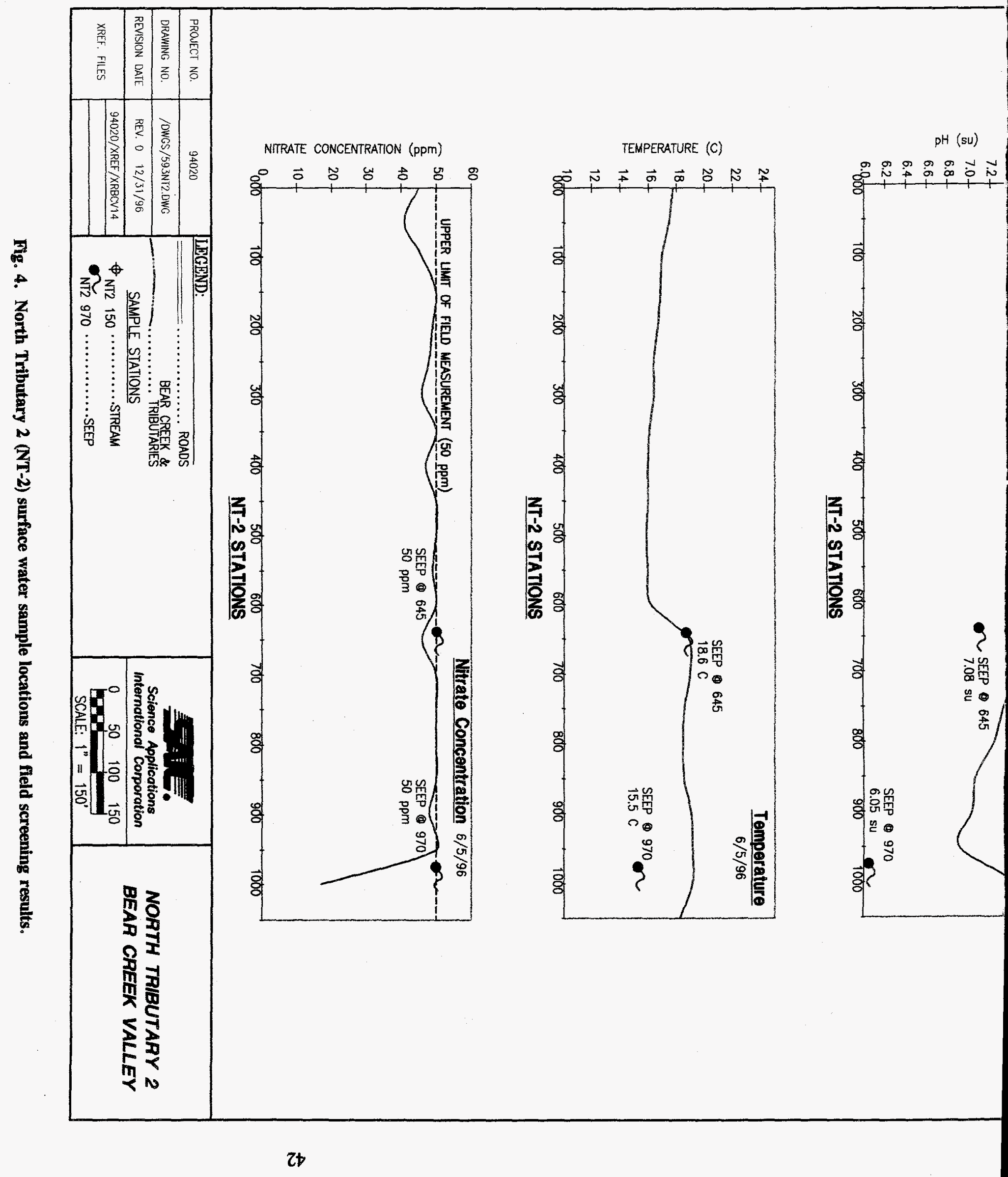




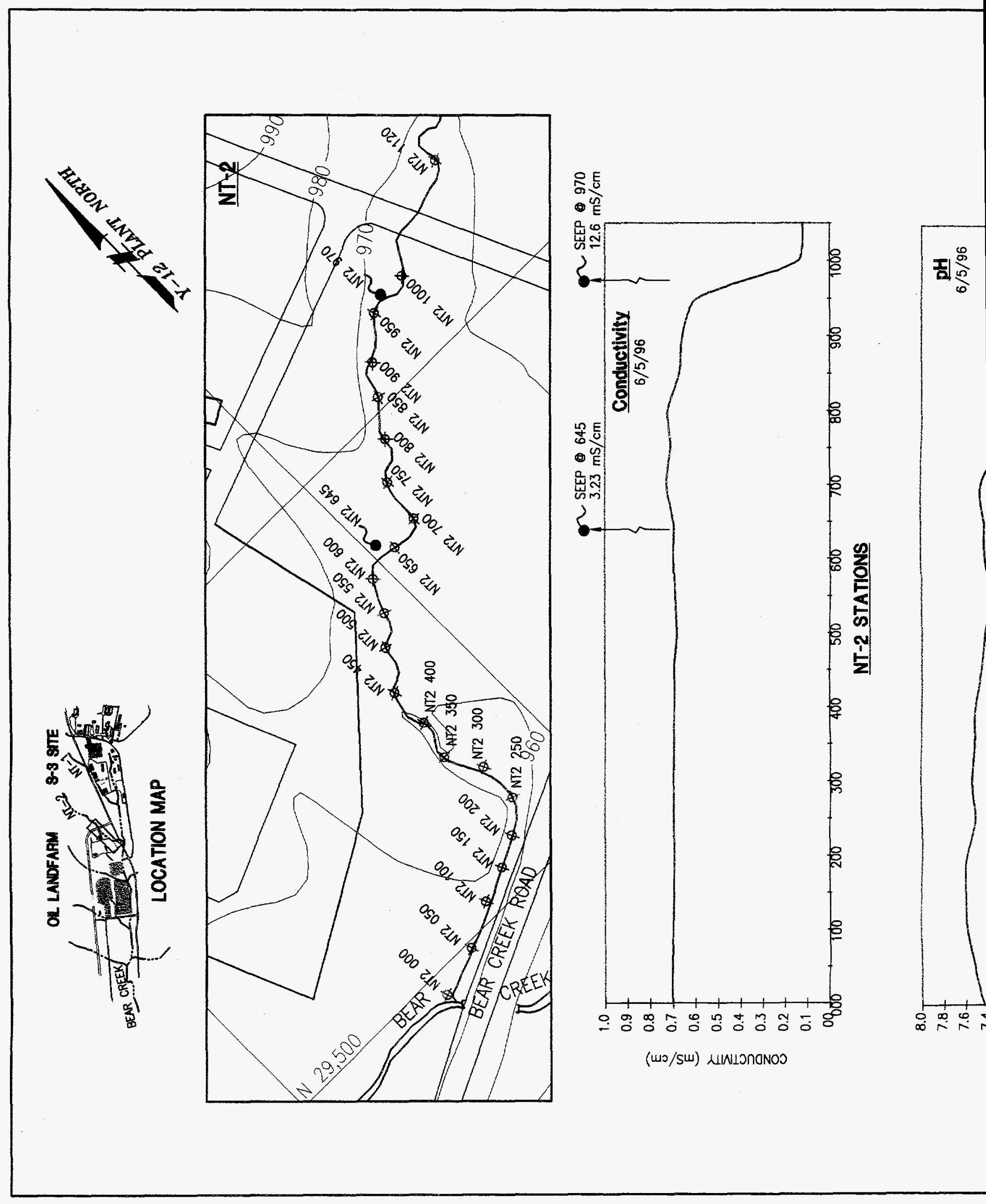




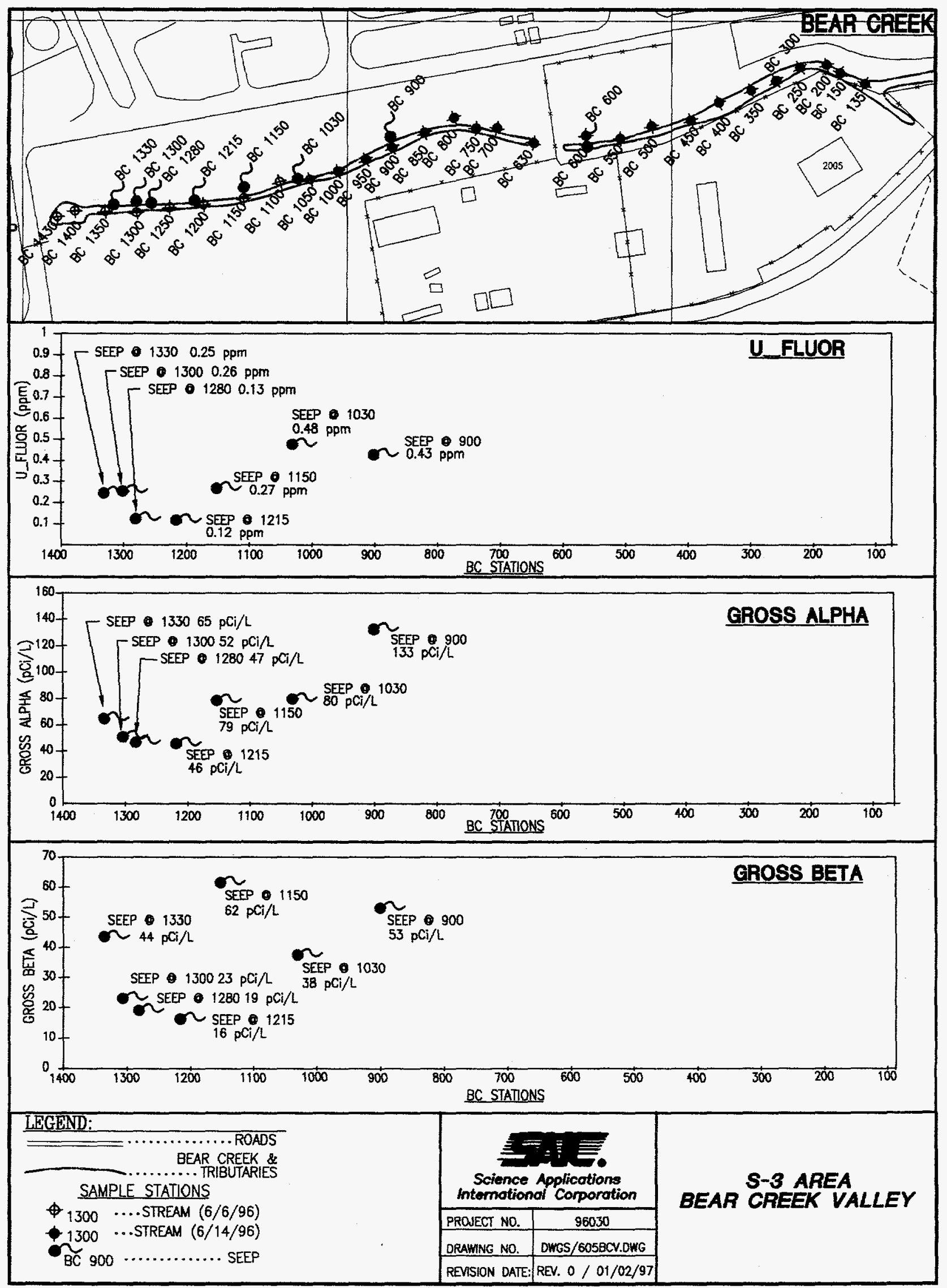

Fig. 5. Bear Creek surface water uranium screening results. 


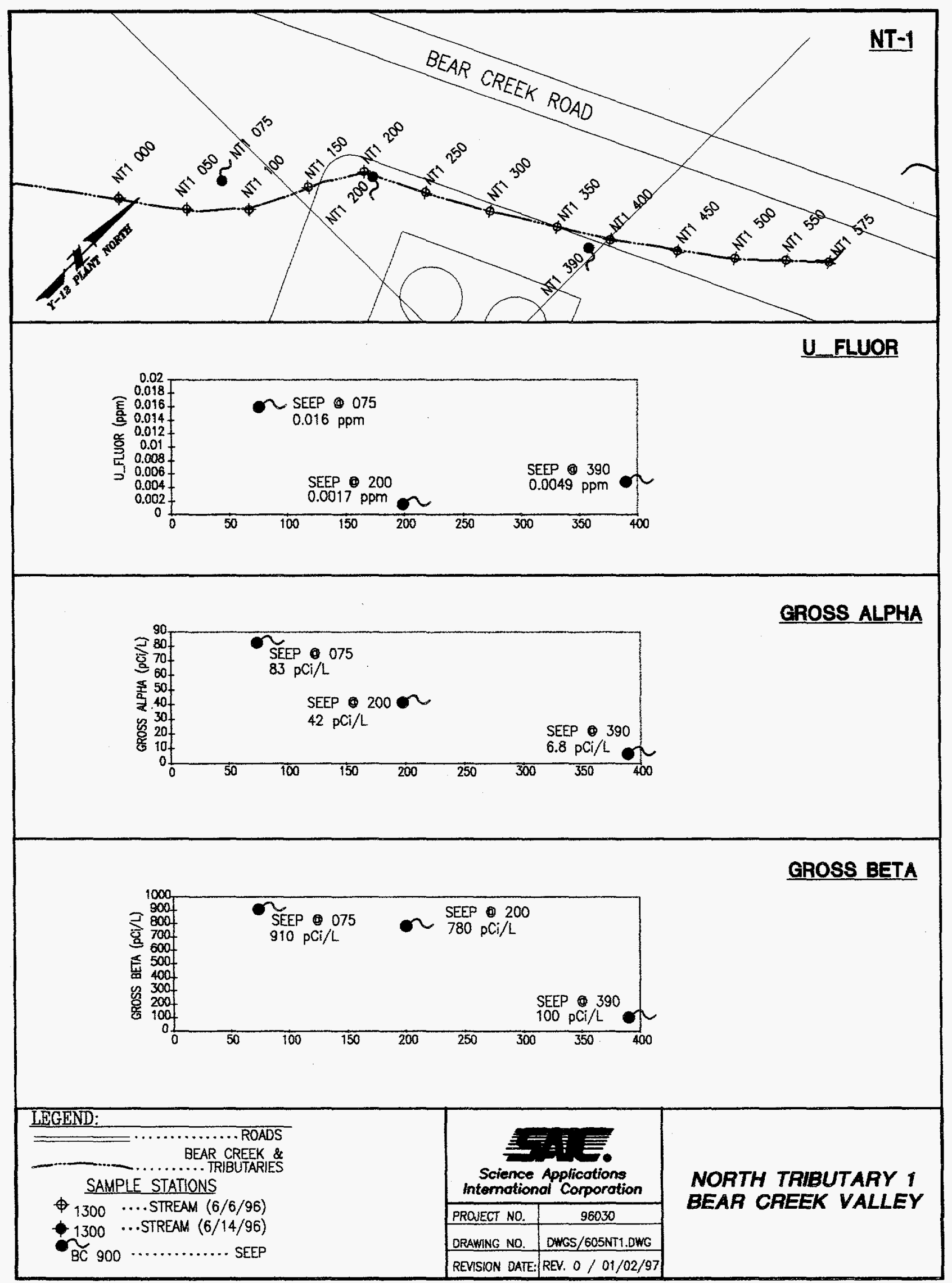

Fig. 6. North Tributary 1 (NT-1) surface water uranium screening results. 


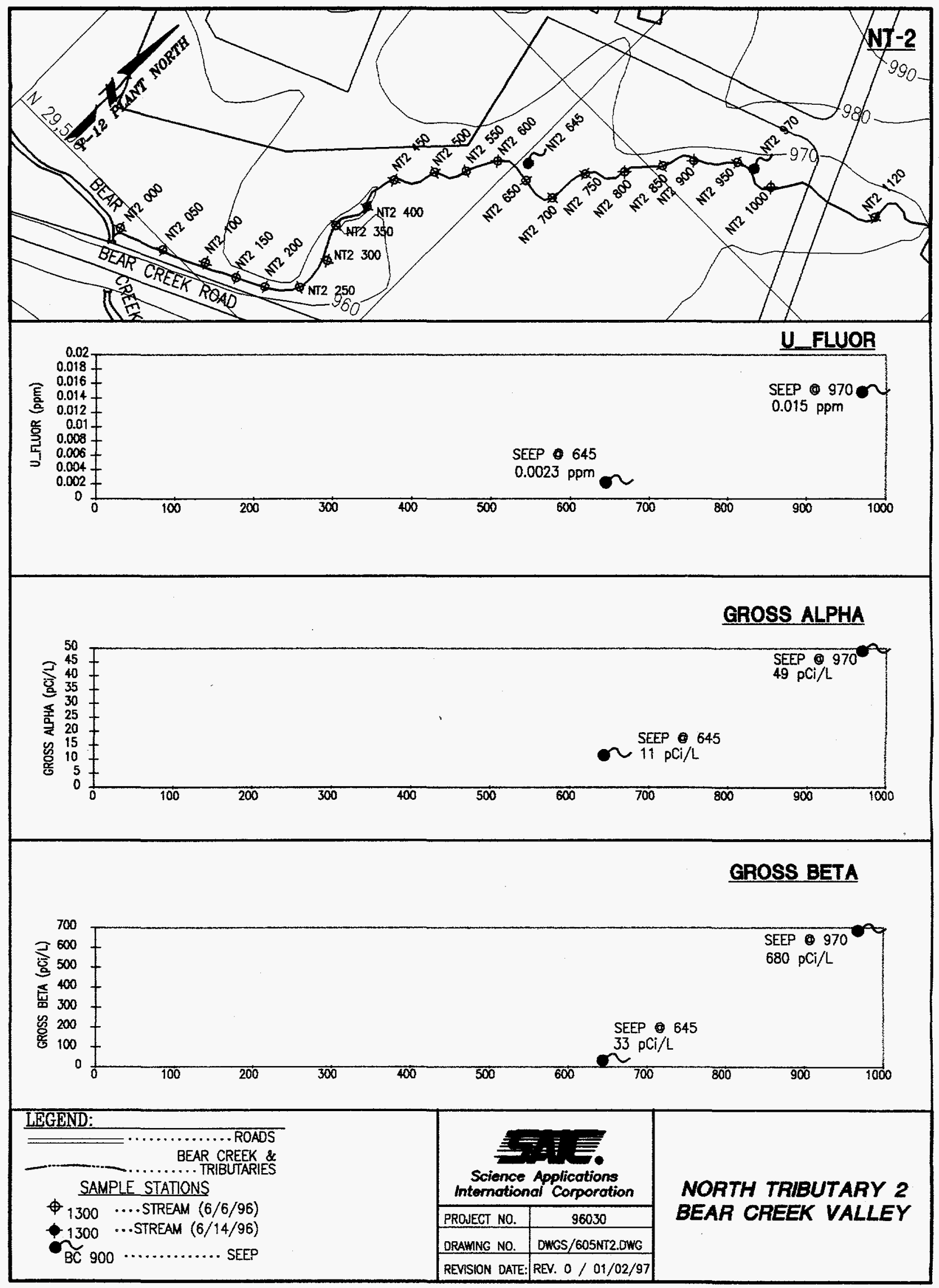

Fig. 7. North Tributary 2 (NT-2) surface water uranium screening results. 



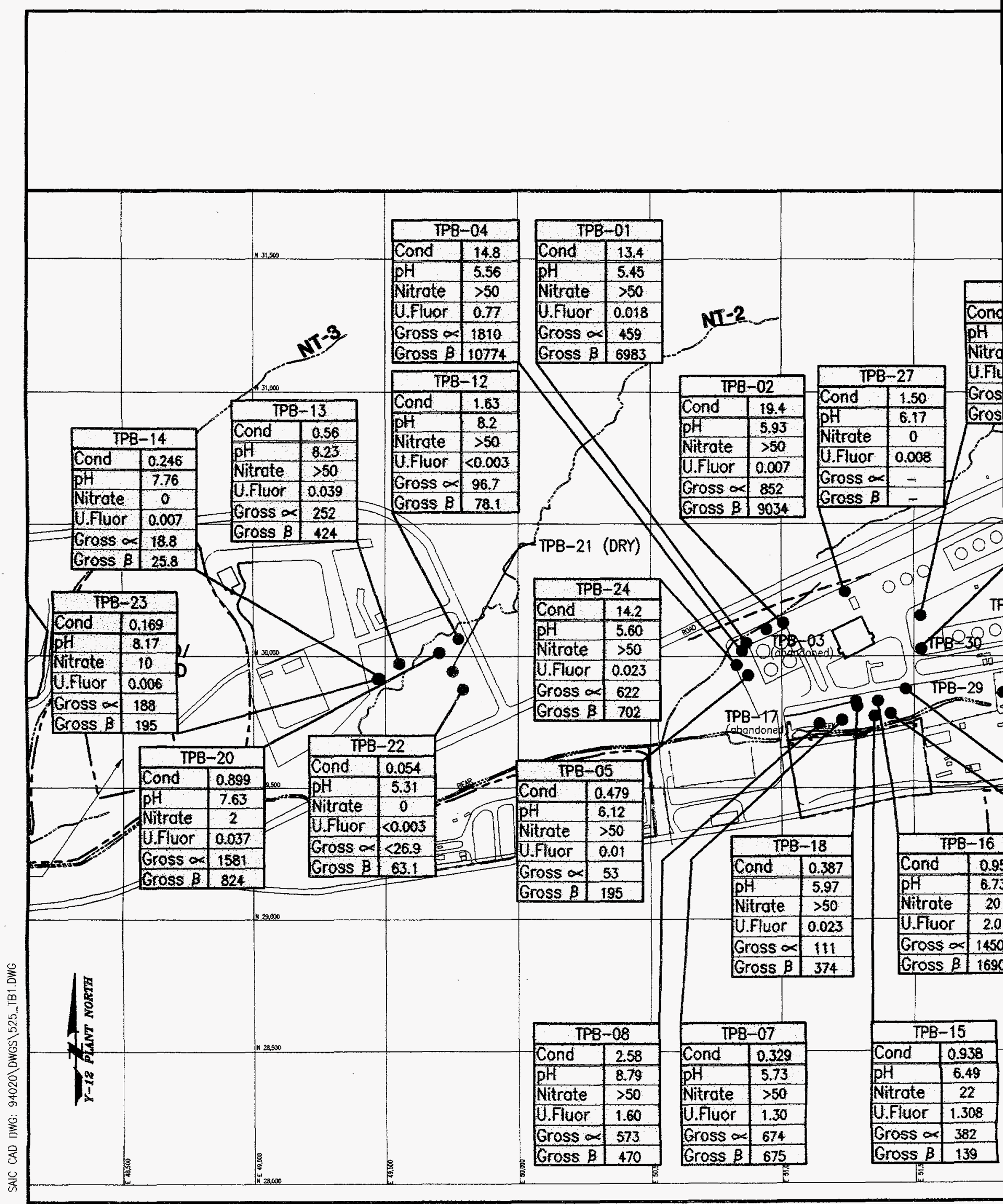

Fig. 8. Push probe temporary piezometer loc 


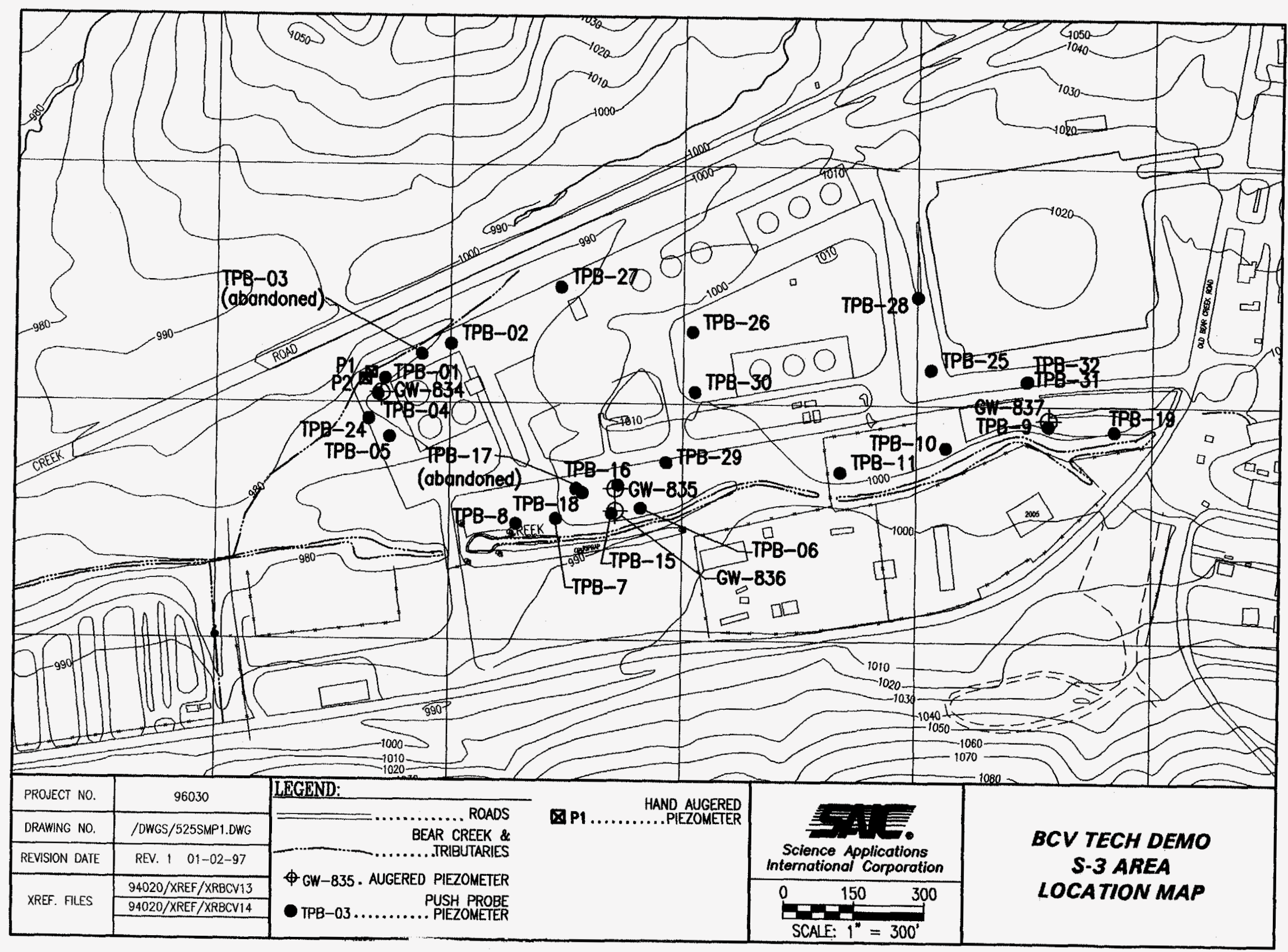

Fig. 9. BCV treatability study S-3 area location map. 


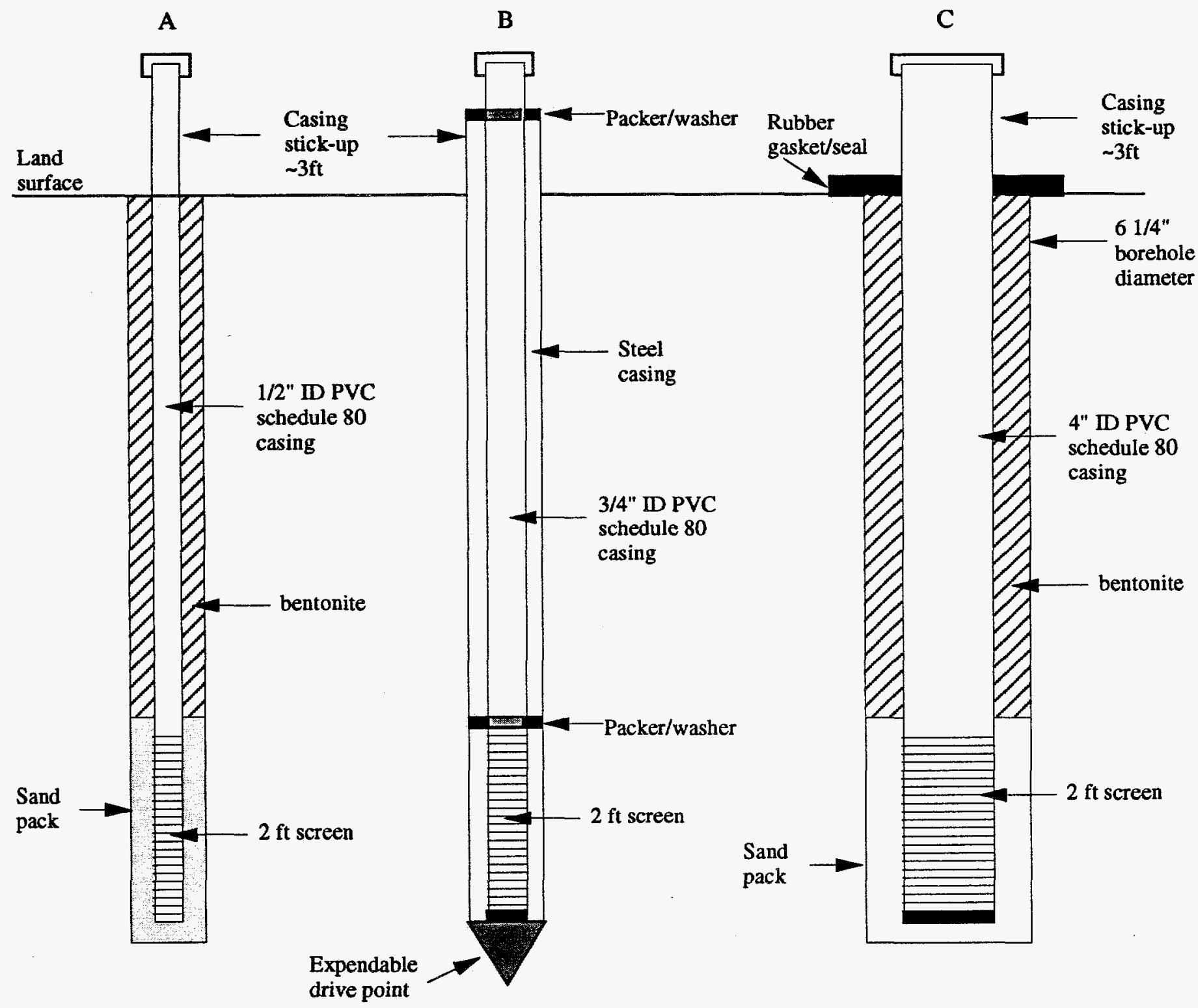

Construction A - TPB-01 through -27

Construction B - TPB-28 through - 32

Construction C - GW-834 through -837

Figure 10. General piezometer construction logs. 


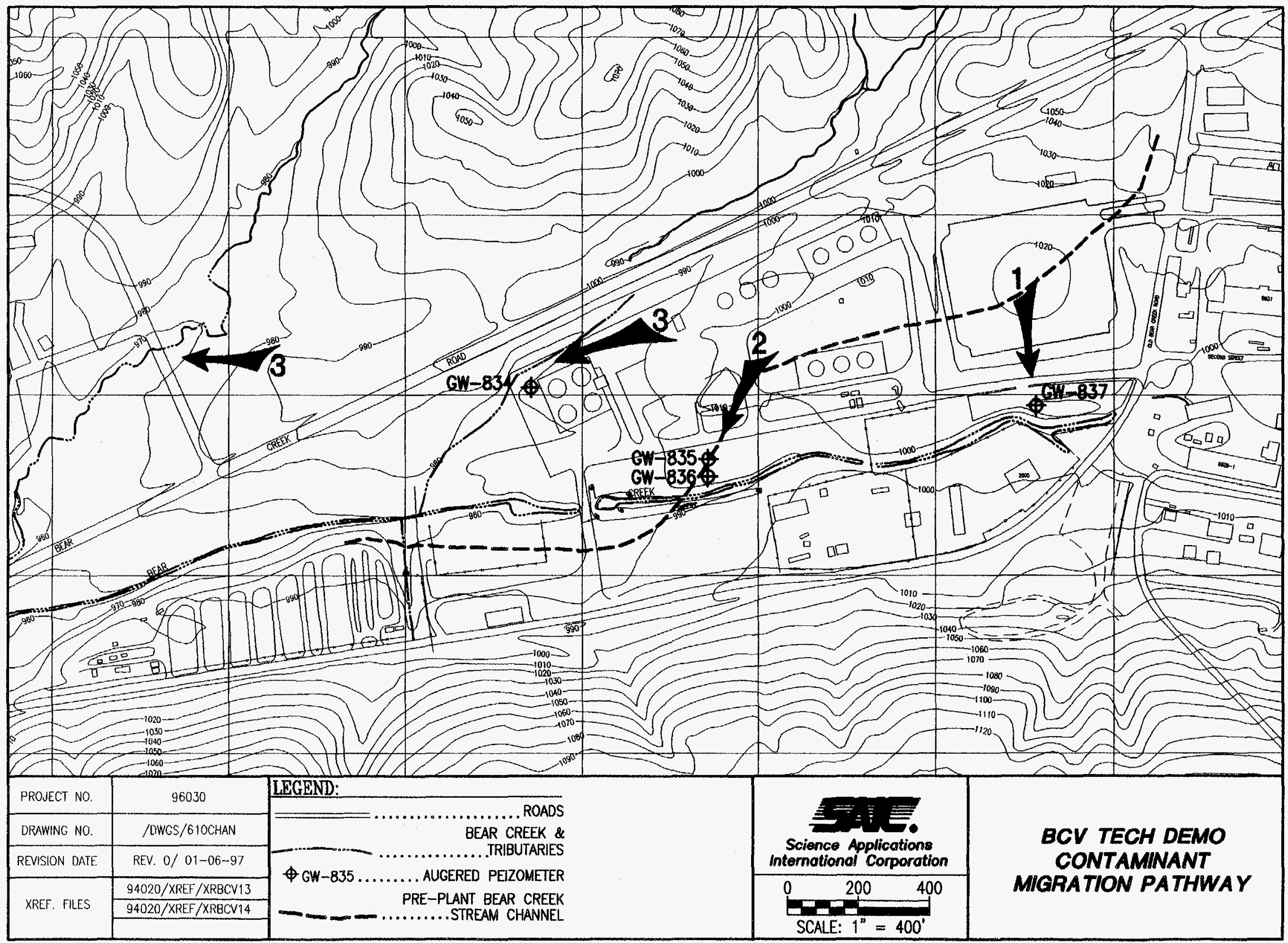

Fig. 11. Contaminant migration pathways. 


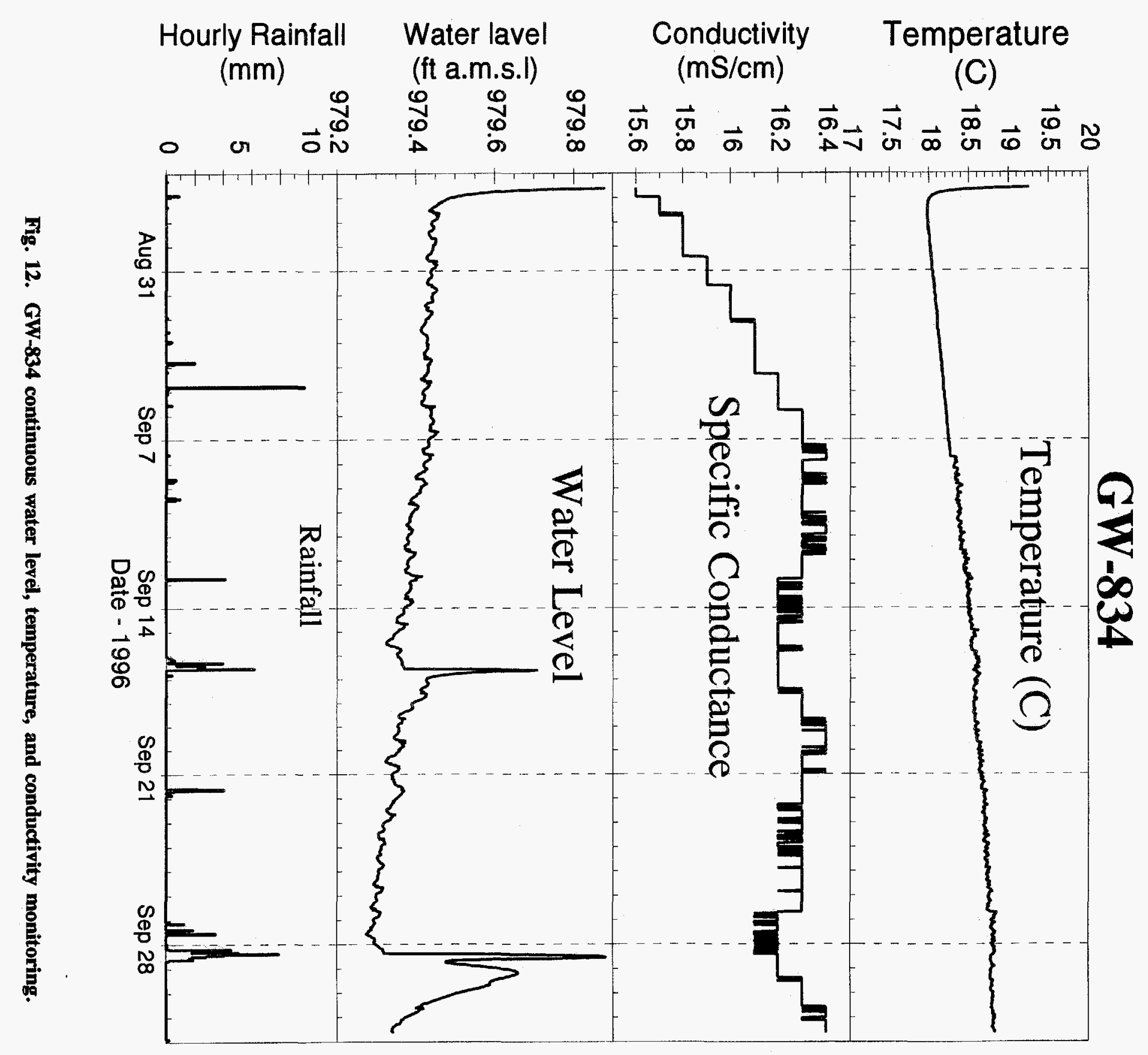




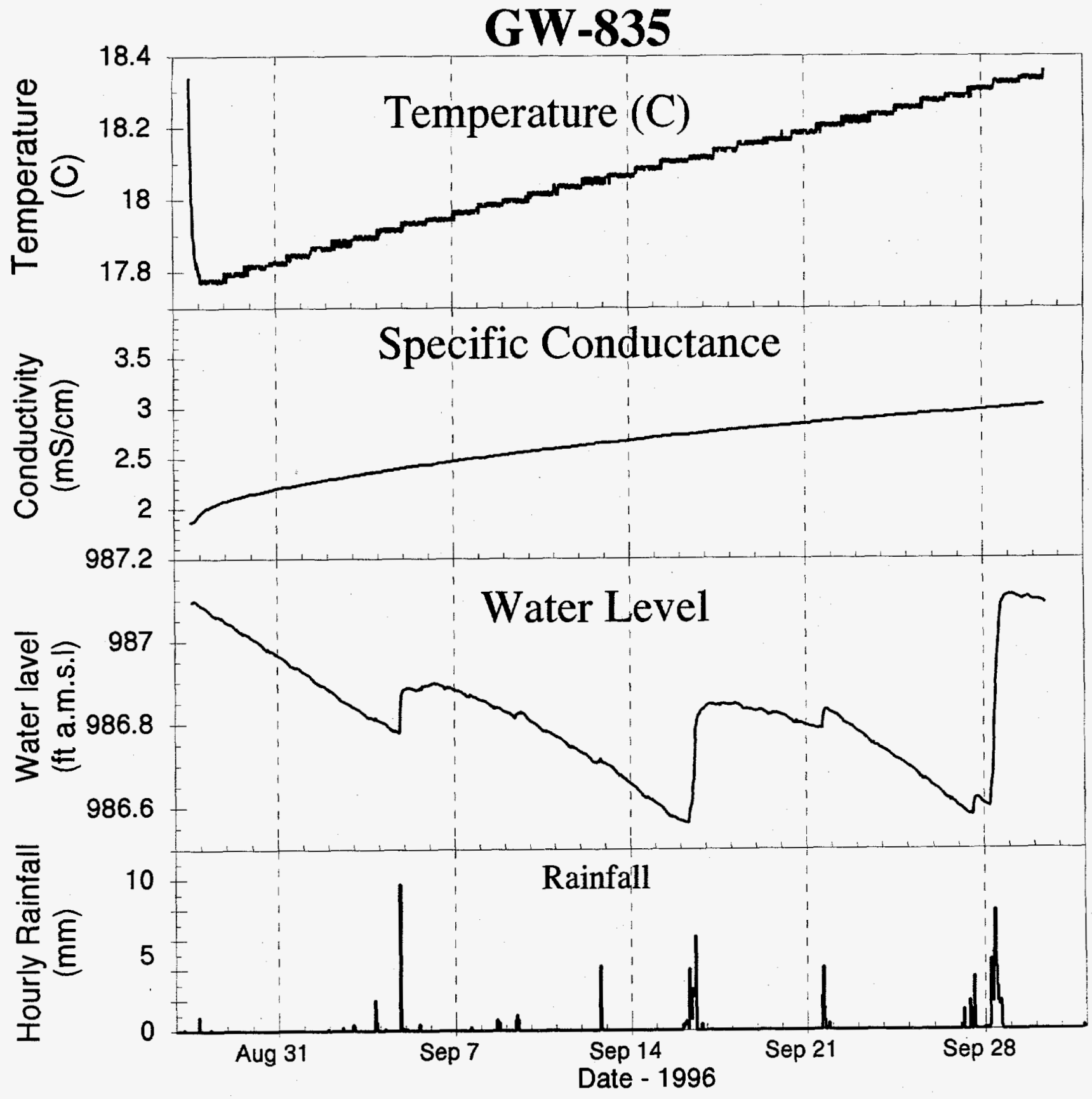

Fig. 13. GW-835 continuous water level, temperature, and conductivity monitoring. 


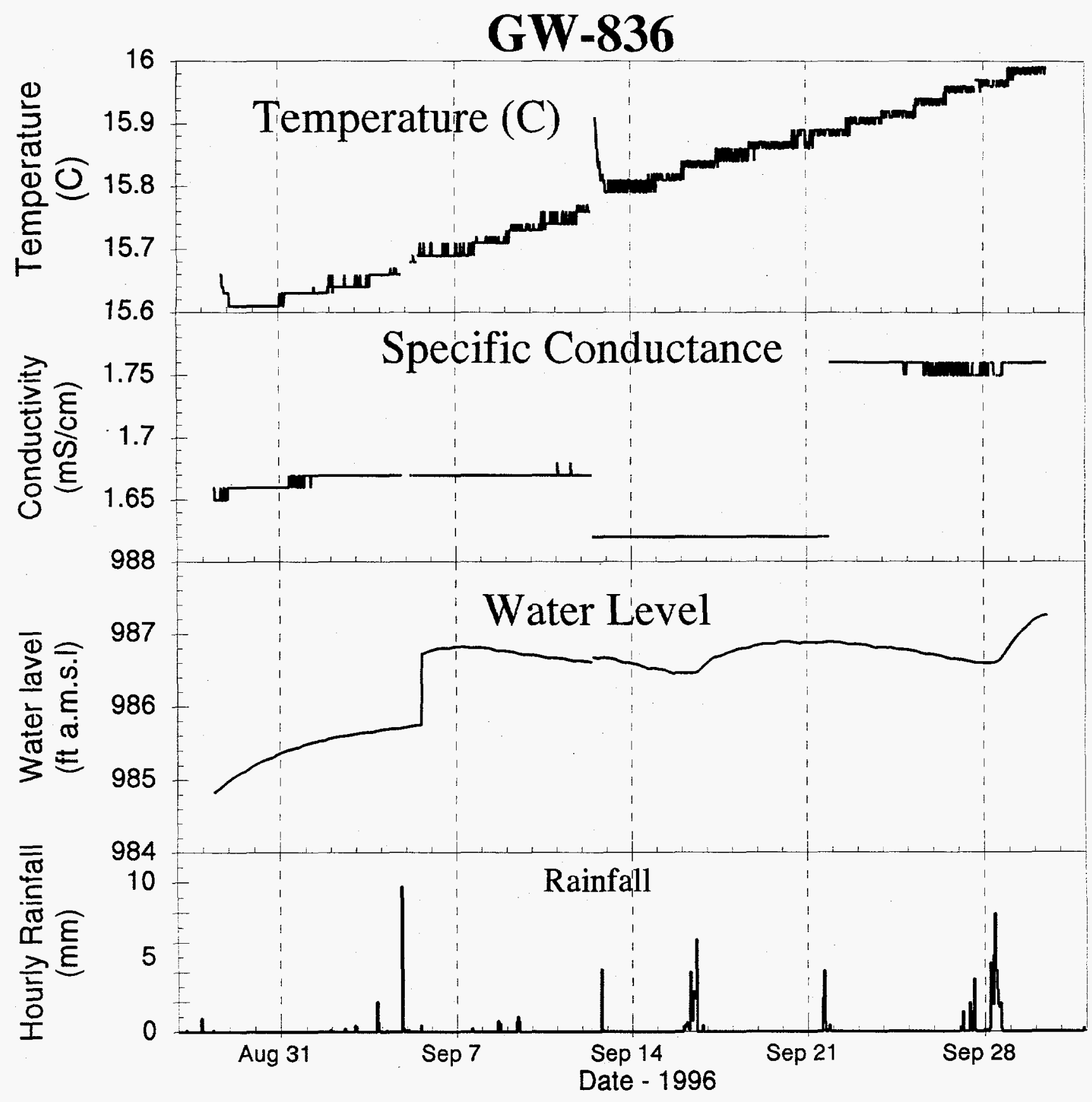

Fig. 14. GW-836 continuous water level, temperature, and conductivity monitoring. 


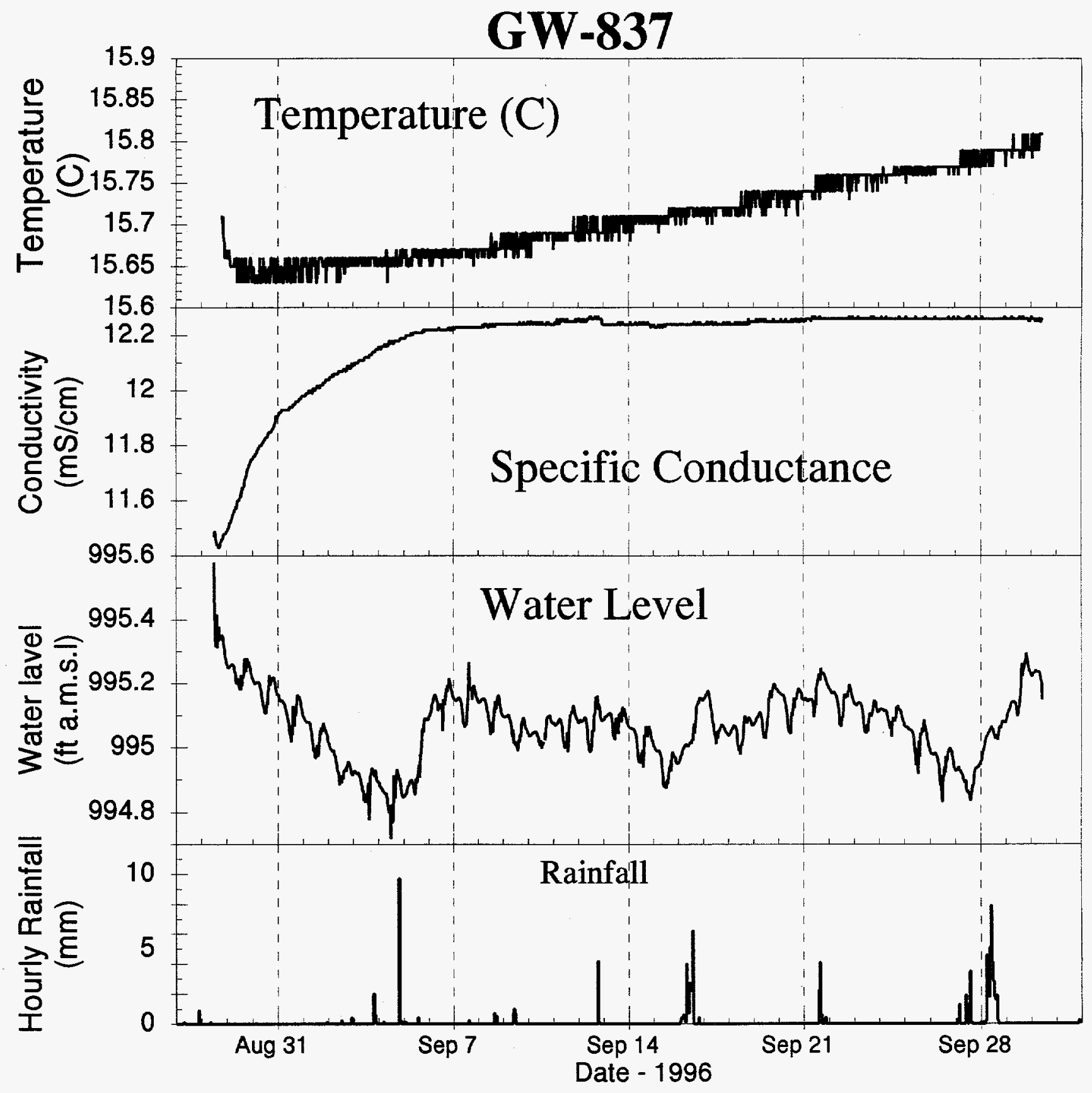

Fig. 15. GW-837 continuous water level, temperature, and conductivity monitoring. 


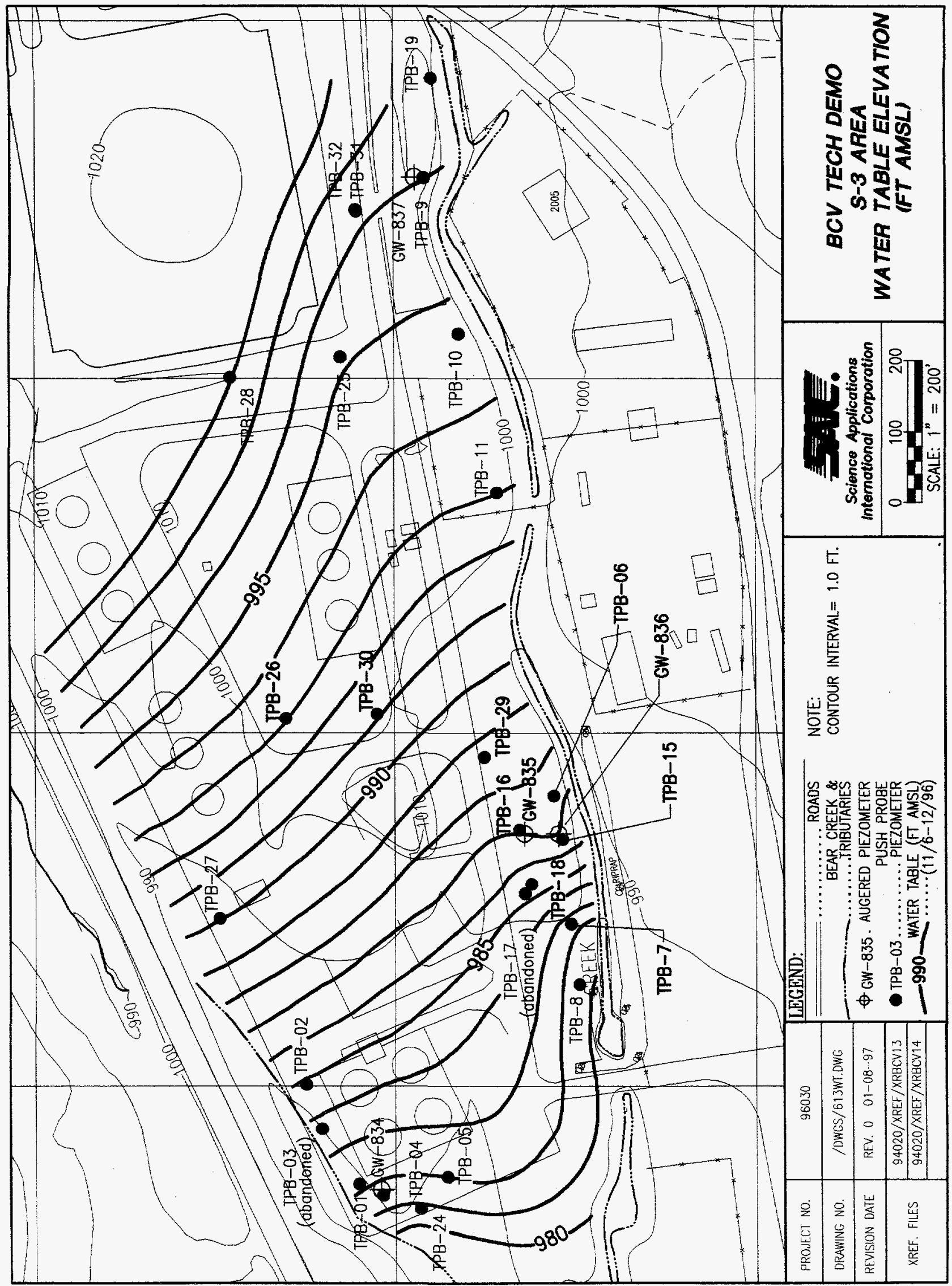

흘 
APPENDIX A

WELL DEVELOPMENT REPORTS

(GW-834, -835, and -837) 

WELL DEVELOPMENT ACTIVITY/PROGRESS REPORT

PAGE 1 of 2

LOCATION: S3 Ponds

BAILERS: G. Shillings/J. Gallaher - Highland Drilling Co HELPERS: NA

DRILL: NA
DATE: START:

FINISH:

8-15-96

LOGGED BY: Timothy Coffey - SAIC

\section{ACTIVITY/COMMENTS}

Arrive at GW-834 site. Don personal protective equipment (PPE).

0944 Arrive at GW-834 site. Don personal protective equipment (PPE)

0948 Enter exclusion zone. Uncap well, organic vapors in casing headspace $=0.0 \mathrm{ppm}$. Measure water level at $10.8 \mathrm{ft}$ below ground surface (BGS). Calculate a well volume of 3.8 gallons.

Commence bailing the well. Bail 4 gallons of water out of the well; water sample is turbid. Breathing zone analysis (BZA) while bailing $=0.0 \mathrm{ppm}$.

Screen water sample. Readings: $\mathrm{pH}=5.8$, conductance $=7600 \mu \mathrm{mhos} / \mathrm{cm}$, temperature $=20.5^{\circ} \mathrm{C}$.

1002 Continue bailing the well. Bail another 2 gallons of water out of the well; water sample is turbid (silty). The well is nearly dry.

1007 Screen water sample. Readings: $\mathrm{pH}=5.6$, conductance $=12,300 \mu \mathrm{mhos} / \mathrm{cm}$, temperature $=19.2^{\circ} \mathrm{C}$.

Wait for well to recharge.

Continue bailing the well. Bail another 2 gallons of water out of the well; water sample is turbid. Well is now dry. 


\section{Y-12 PLANT GROUNDWATER PROTECTION PROGRAM}

WELL NO. GW-834

\section{WELL DEVELOPMENT ACTIVITYIPROGRESS REPORT continued}

PAGE 2 of 2

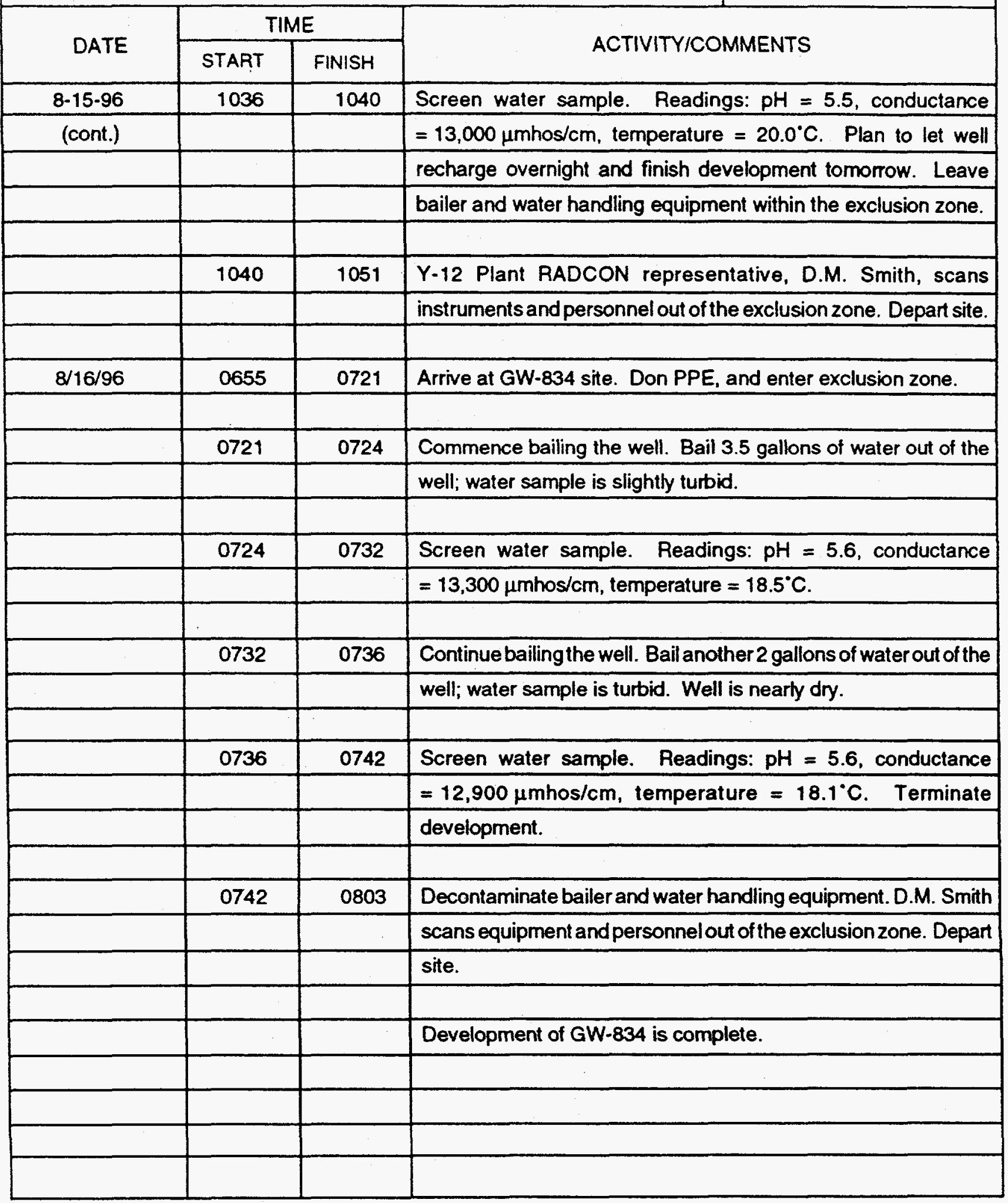




\section{Y-12 PLANT GROUNDWATER PROTECTION PROGRAM}

\section{MONITORING WELL DEVELOPMENT PROGRESS}

WELL NO. GW-834

LOCATION: S3 Ponds

ONE WELL VOLUME: $\quad 3.8$

GALLONS

WELL INSTALLATION

COMPLETION DATE:

$7-11-96$

\begin{tabular}{|c|c|c|c|c|c|c|c|}
\hline DATE & $\begin{array}{l}\text { GALLONS } \\
\text { PUMPED }\end{array}$ & $\begin{array}{l}\text { DESCRIPTION } \\
\text { OF TURBIDITY }\end{array}$ & PH & $\begin{array}{l}\text { DISSOLVED } \\
\text { SOUDS (uS) }\end{array}$ & $\begin{array}{l}\text { TOTAL } \\
\text { OALONS } \\
\text { PUMPED } \\
\end{array}$ & $\begin{array}{c}\text { WELL } \\
\text { VOLUMES } \\
\text { PUMPEDD }\end{array}$ & COMMENTS \\
\hline $8-15-96$ & 4 & Turbid & 5.8 & 7600 & 4 & 1.1 & \\
\hline $8-15-96$ & 2 & Turbid (Silty) & 5.6 & 12,300 & 6 & 1.6 & \\
\hline $8-15-96$ & 2 & Turbid & 5.5 & 13,000 & 8 & 2.1 & 1. \\
\hline $8-16-96$ & 3.5 & Slightly Turbid & 5.6 & 13,300 & 11.5 & 3.0 & \\
\hline $8-16-96$ & 2 & Turbid & 5.6 & 12,900 & 13.5 & 3.6 & 1. \\
\hline & & & & & & & \\
\hline & & & & & & & \\
\hline & & & & & & & \\
\hline & & & & & & & \\
\hline & & & & & & & \\
\hline & & & & & & & \\
\hline & & & & & & & \\
\hline & & & & & & & \\
\hline & & & & & & & \\
\hline & & & & & & & \\
\hline & & & & & & & \\
\hline & & & & & & & \\
\hline & & & & & & & \\
\hline & & & & & & & \\
\hline \multirow{2}{*}{\multicolumn{2}{|c|}{$\begin{array}{l}\text { RESULTS AT END } \\
\text { OF DEVELOPMENT } \\
\text { COMMENTS }\end{array}$}} & Turbid & 5.6 & 12,900 & 13.5 & 3.6 & \\
\hline & & & & & & & \\
\hline
\end{tabular}




\section{Y-12 PLANT GROUNDWATER PROTECTION PROGRAM}

WELL NO. GW-834

\section{MONITORING WELL DEVELOPMENT SUMMARY}

METHOD OF DEVELOPMENT: 4 -liter bailer

DEVELOPMENT OBSERVED BY: Timothy Coffey - SAIC

ONE WELL VOLUME: 3.8

TOTAL GALLONS PUMPED: 13.5

INITIAL pH: 5.8 FINAL pH: $7600 \mu \mathrm{mhos} / \mathrm{cm}$ DEVELOPMENT DATE: START: $8-15-96$

FINISH: $8-16-96$

INITIAL SPECIFIC CONDUCTANCE: GALLONS TOTAL WELL VOLUMES PUMPED: 3.6 5.6

DESCRIPTION OF INITIAL TURBIDITY:

DESCRIPTION OF FINAL TURBIDITY:

FINAL MEASURED TURBIDITY:

WELL APPROVED BY: Turbid Turbid Not Analyzed

W.K. Jago - HSEA

ODOR OF WATER: None discernable

WATER DISCHARGED TO: $\square$ GROUND SURFACE $\square$ STORM SEWERS $\square$ DRUMS

$\square$ TANK TRUCK

X STORAGE TANKS

$\square$ OTHER

INITIAL PRE-DEVELOPMENT

WATER DEPTH: 10.8 tt BGS on 8-15-96

DEVELOPMENT OBSERVATIONS: Well is a moderate to fair producer. Well always gave more than 1 well volume prior to drying up. Water cleared up fairly well, however, the last (sediment-laden) sample was turbid due to the higher ratio of sediments to water as the well dried up. 
WELL NO.

GW-834

SITE:

S3 Ponds

APPROX. VOLUME OF DEVELOPMENT WATER:

13.5 gallons

CALIBRATION OF INSTRUMENTS (check those instruments calibrated to manufacturer's specifications):

pH meter

Sp. Cond. meter

Organic vapor meter

Beta/gamma meter

Alpha meter

$\frac{\frac{x}{x}}{\frac{x}{x}} \frac{x}{x}$

(model)

(model)

(model)

(model)

(model)
Horiba Model U-7

YSI S-C-T Meter Model 33

Century/Foxboro OVA Model 128

Ludlum Model 3 Survey Meter with G-M Pancake Probe

Ludlum Model 12 Count Ratemeter

with Scintillation Tube Probe

FIELD SCREENING RESULTS:

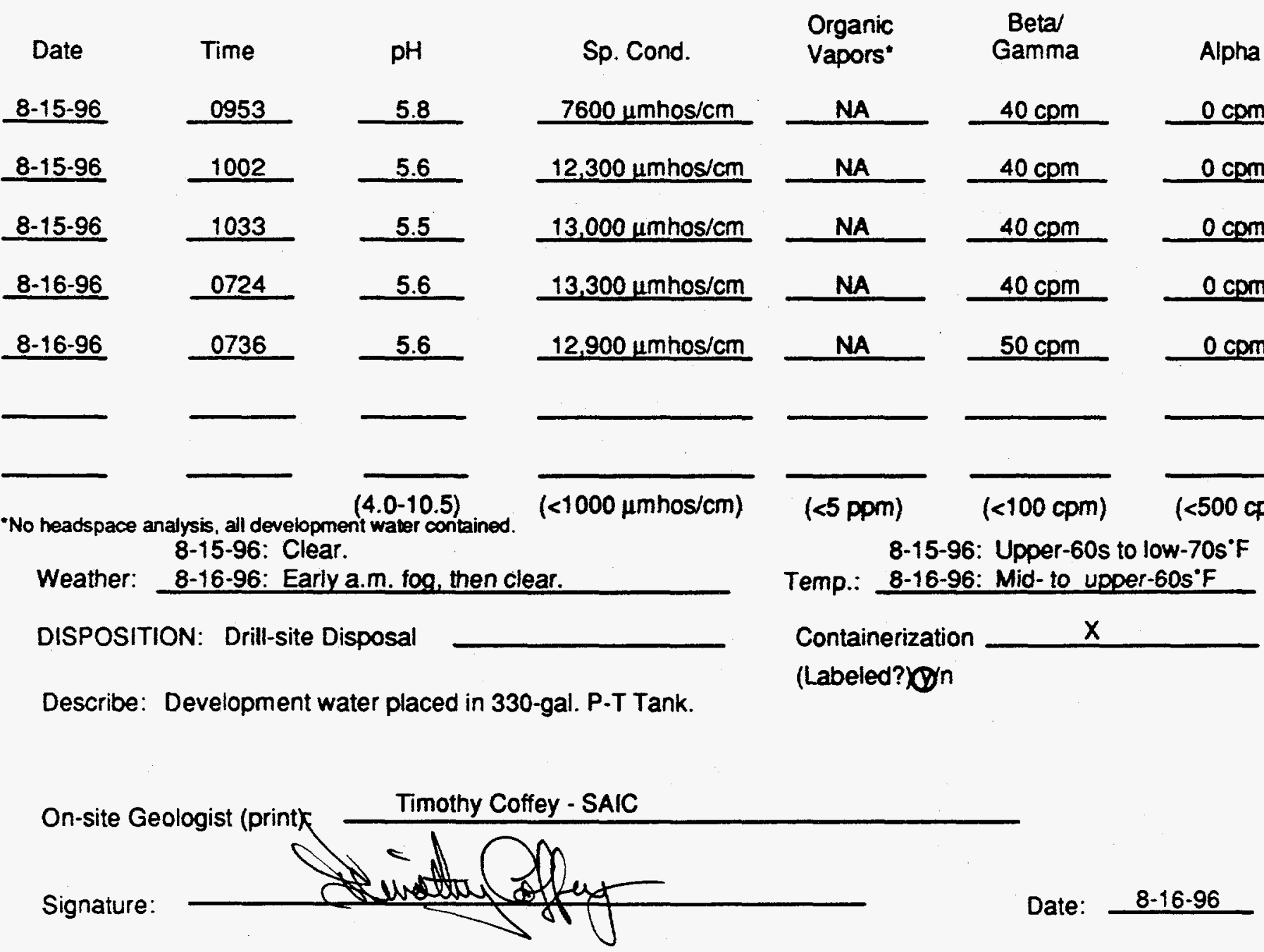




\section{Y-12 PLANT GROUNDWATER PROTECTION PROGRAM}

\section{EQUIPMENT DECONTAMINATION INSPECTION SUMMARY}

LOCATION: S3 Ponds

DECONTAMINATION CREW: G. Shillings
WELL NO. GW-834

INSTALLATION P\&A

DEVELOPMENT
DATE: START: $8-15-96$

FINISH: 8-16-96

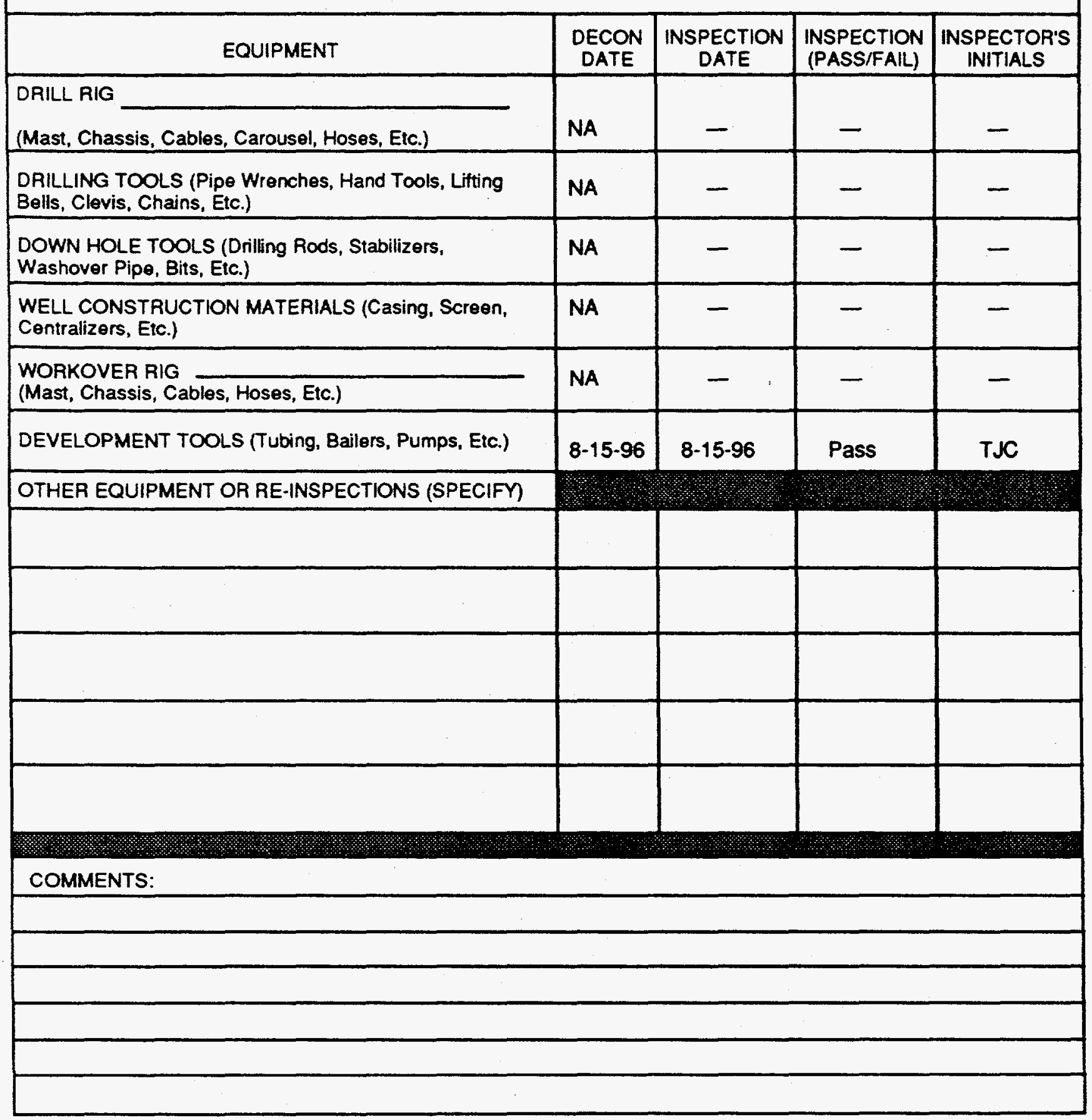




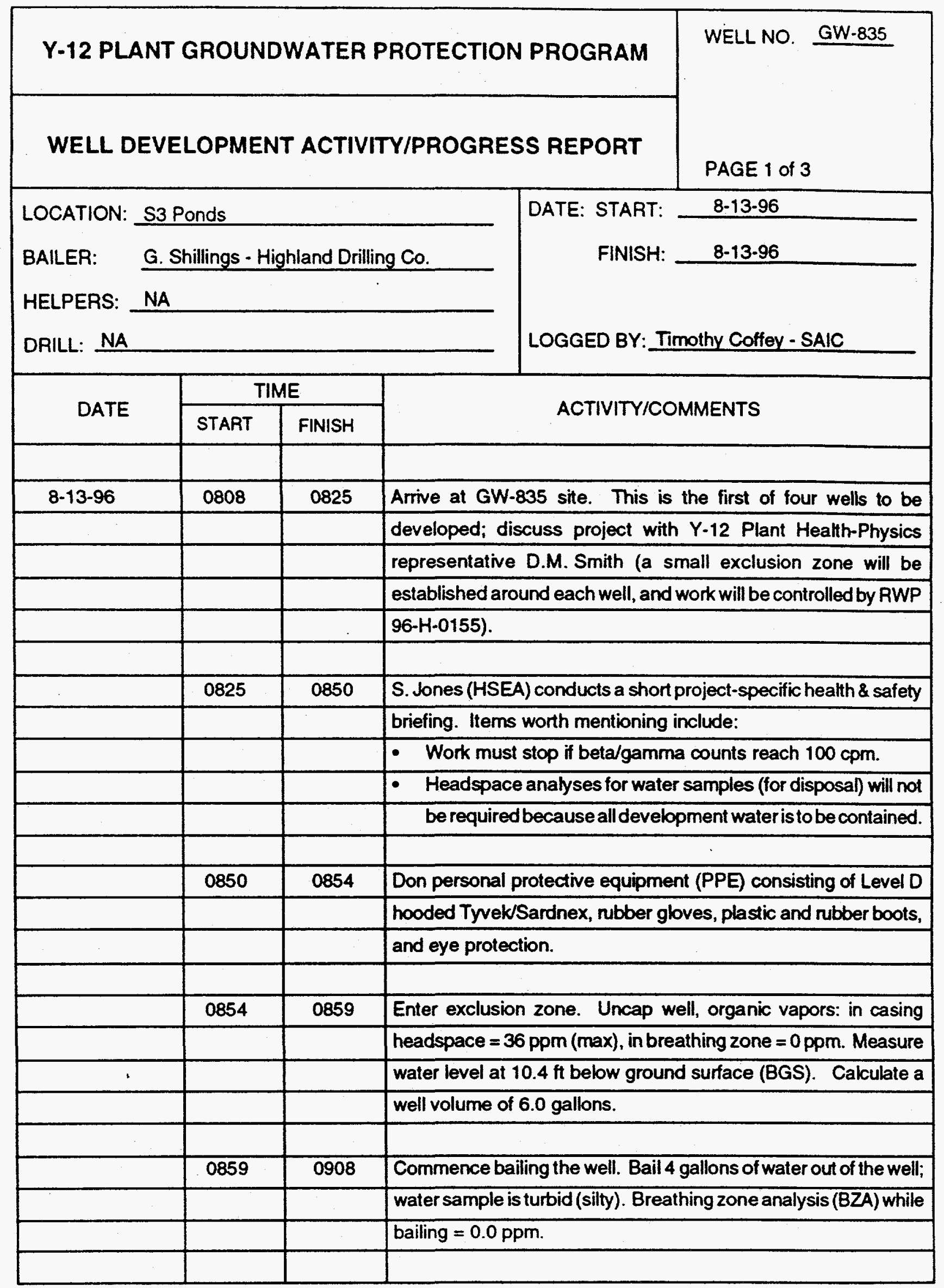




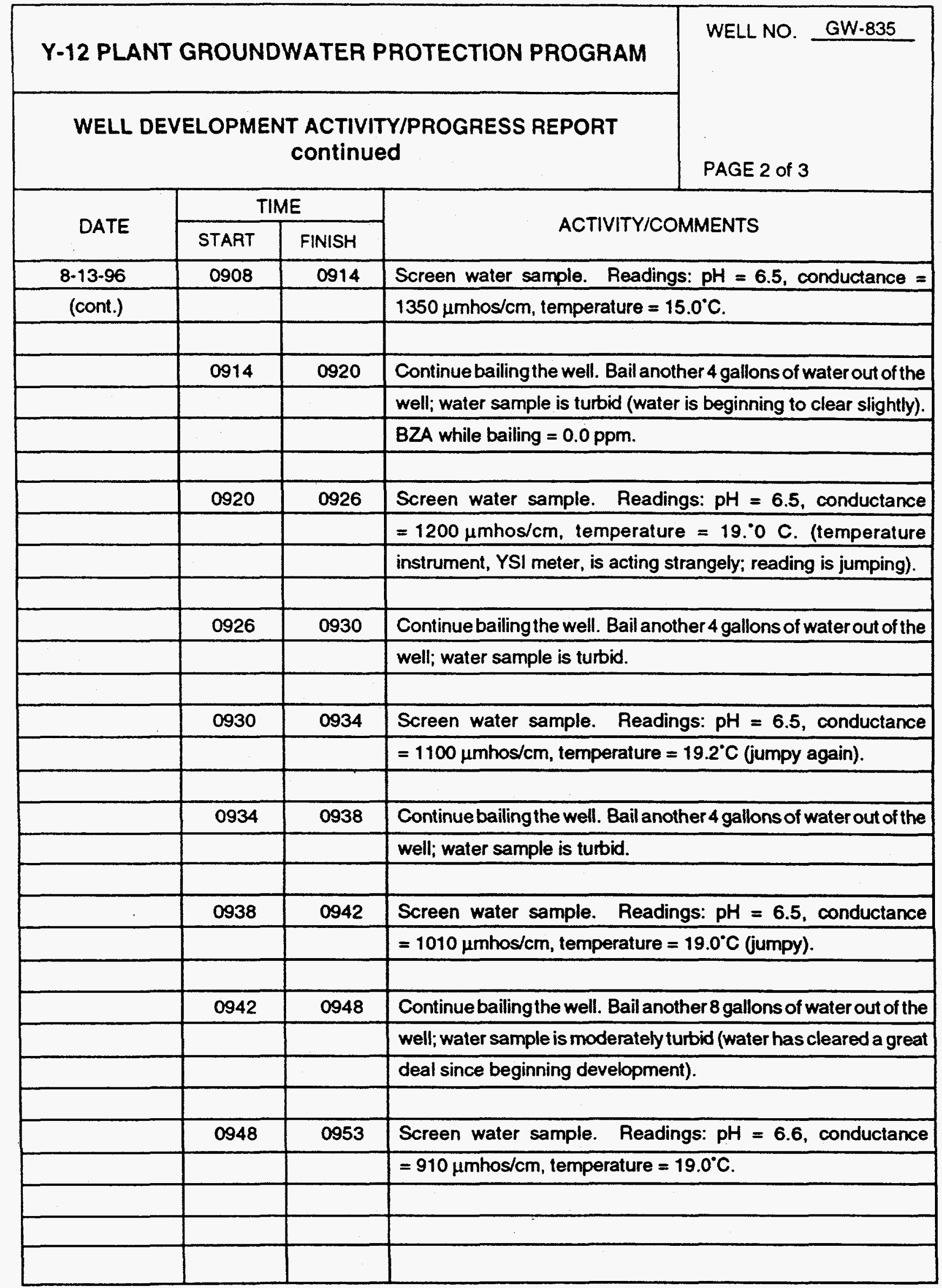




\begin{tabular}{|c|c|c|c|c|}
\hline \multicolumn{4}{|c|}{ Y-12 PLANT GROUNDWATER PROTECTION PROGRAM } & \multirow{2}{*}{$\begin{array}{l}\text { WELL NO. GW.835 } \\
\text { PAGE } 3 \text { of } 3\end{array}$} \\
\hline \multicolumn{4}{|c|}{$\begin{array}{l}\text { WELL DEVELOPMENT ACTIVITY/PROGRESS REPORT } \\
\text { continued }\end{array}$} & \\
\hline \multirow{2}{*}{ DATE } & \multicolumn{2}{|c|}{ TIME } & \multirow{2}{*}{\multicolumn{2}{|c|}{ ACTIVITY/COMMENTS }} \\
\hline & START & FINISH & & \\
\hline 8-13-96 & 0953 & 0959 & Continue bailing the well. Bail a & er 8 gallons of water out of the \\
\hline \multirow[t]{4}{*}{ (cont.) } & & & \multicolumn{2}{|c|}{ well; water sample is moderately turbid. First sign that well is } \\
\hline & & & \multicolumn{2}{|c|}{ beginning to dry up; water level has dropped significantly. } \\
\hline & 0959 & 1004 & \multicolumn{2}{|c|}{ Screen water sample. Readings: $\mathrm{pH}=6.5$, conductance } \\
\hline & & & \multicolumn{2}{|c|}{$=980 \mu \mathrm{mhos} / \mathrm{cm}$, temperature $=19.0^{\circ} \mathrm{C}$} \\
\hline & 1004 & 1011 & \multicolumn{2}{|c|}{ Continue bailing the well. Bail another 8 gallons of water out of the } \\
\hline & & & \multicolumn{2}{|c|}{ well; water sample is slightly turbid. Water continues to clear, while } \\
\hline & & & \multicolumn{2}{|c|}{ water level continues to drop. } \\
\hline & 1011 & 1015 & \multicolumn{2}{|c|}{ Screen water sample. Readings: $\mathrm{pH}=6.6$, Conductance } \\
\hline & & & \multicolumn{2}{|c|}{$=890 \mu \mathrm{mhos} / \mathrm{cm}$, temperature $=19.0^{\circ} \mathrm{C}$} \\
\hline & 1015 & 1023 & \multicolumn{2}{|c|}{ Continue bailing the well. Bail another 8 gallons of water out of the } \\
\hline & & & \multicolumn{2}{|c|}{ well; water sample is slightly turid. } \\
\hline & 1023 & 1027 & \multicolumn{2}{|c|}{ Screen water sample. Readings: $\mathrm{pH}=6.6$, conductance } \\
\hline & & & \multicolumn{2}{|c|}{$=890 \mu \mathrm{mhos} / \mathrm{cm}$, temperature $=19.0^{\circ} \mathrm{C}$} \\
\hline & 1027 & 1055 & \multicolumn{2}{|c|}{ S. Jones (HSEA) terminates development of GW-835: have } \\
\hline & & & \multicolumn{2}{|c|}{ removed 8.0 well volumes, parameters have stabilized and water } \\
\hline & & & \multicolumn{2}{|c|}{ has cleared significantly. Decontaminate bailer and other water } \\
\hline & & & \multicolumn{2}{|c|}{ handling items. D.M. Smith scans equipment and personnel out } \\
\hline & & & \multicolumn{2}{|l|}{ of the exclusion zone. } \\
\hline & 1055 & 1101 & \multicolumn{2}{|l|}{ Pack up equipment. Depart site. } \\
\hline & & & \multicolumn{2}{|c|}{ Development of GW-835 is complete. } \\
\hline & & & & \\
\hline & & & & \\
\hline & & & & \\
\hline
\end{tabular}




\section{Y-12 PLANT GROUNDWATER PROTECTION PROGRAM}

WELL NO. GW-835

\section{MONITORING WELL DEVELOPMENT PROGRESS}

LOCATION: S3 PONdS

ONE WELL VOLUME: 6.0 GALLONS

WELL INSTALLATION

COMPLETION DATE:

\section{$7 \cdot 11.96$}

\begin{tabular}{|c|c|c|c|c|c|c|c|}
\hline DATE & $\begin{array}{l}\text { GALLONS } \\
\text { PUMPED }\end{array}$ & $\begin{array}{l}\text { DESCRIPTION } \\
\text { OF TURBIDITY }\end{array}$ & pH & $\begin{array}{l}\text { DISSOLVED } \\
\text { SOLDS (uSS) }\end{array}$ & $\begin{array}{l}\text { TOTAL } \\
\text { GALLONS } \\
\text { PUMPEED }\end{array}$ & $\begin{array}{l}\text { WELL } \\
\text { VOLUMES } \\
\text { PUMPED }\end{array}$ & COMMENTS \\
\hline $8-13-96$ & 4 & Turbid (silty) & 6.5 & 1350 & 4 & 0.7 & \\
\hline $8-13-96$ & 4 & Turbid & 6.5 & 1200 & 8 & 1.3 & \\
\hline $8-13-96$ & 4 & Turbid & 6.5 & 1100 & 12 & 2.0 & \\
\hline $8-13-96$ & 4 & Turbid & 6.5 & 1010 & 16 & 2.7 & \\
\hline $8-13-96$ & 8 & Moderately Turbid & 6.6 & 910 & 24 & 4.0 & \\
\hline $8-13-96$ & 8 & Moderately Turbid & 6.5 & 980 & 32 & 5.3 & \\
\hline $8-13-96$ & 8 & Slightly Turbid & 6.6 & 890 & 40 & 6.7 & \\
\hline 8-13-96 & 8 & Slightly Turbid & 6.6 & 890 & 48 & 8.0 & \\
\hline & & & & & & & \\
\hline & & & & & & & \\
\hline & & & & & & & \\
\hline & & & & & & & \\
\hline & & & & & & & \\
\hline & & & & & & & \\
\hline & & & & & & & \\
\hline & & & & & & & \\
\hline & & & & & & & \\
\hline & & & & & & & \\
\hline & & & & & & & \\
\hline \multicolumn{2}{|c|}{$\begin{array}{l}\text { RESULTS AT END } \\
\text { OF DEVELOPMENT }\end{array}$} & Slightly Turbid & 6.6 & 890 & 48 & 8.0 & \\
\hline \multicolumn{8}{|c|}{ COMMENTS: } \\
\hline & & & & & & & \\
\hline & & & & & & & \\
\hline & & & & & & & \\
\hline & & & & & & & \\
\hline & & & & & & & \\
\hline & & & & & & & \\
\hline
\end{tabular}




\section{Y-12 PLANT GROUNDWATER PROTECTION PROGRAM}

WELL NO. GW-835

\section{MONITORING WELL DEVELOPMENT SUMMARY}

METHOD OF DEVELOPMENT: 4-liter bailer

DEVELOPMENT OBSERVED BY: Timothy Coffey - SAIC

ONE WELL VOLUME: 6.0 GALLONS

TOTAL GALLONS PUMPED: 48 TOTAL WELL VOLUMES PUMPED:

8.0

INITIAL pH: 6.5 FINAL pH: 6.6

INITIAL SPECIFIC CONDUCTANCE: $1350 \mu \mathrm{mhos} / \mathrm{cm}$ FINAL: $890 \mu \mathrm{mhos} / \mathrm{cm}$

DESCRIPTION OF INITIAL TURBIDITY:

DESCRIPTION OF FINAL TURBIDITY:

Turbid (silty)

FINAL MEASURED TURBIDITY:

Slightly Turbid

WELL APPROVED BY:

Not Analyzed

W.K. Jago - HSEA

ODOR OF WATER: None Discernable

WATER DISCHARGED TO:

$\square$ GROUND SURFACE
$\square$ STORM SEWERS
$\square$ DRUMS

TANK TRUCK

X STORAGE TANKS

$\square$ OTHER

INITIAL PRE-DEVELOPMENT

WATER DEPTH: $10.4 \mathrm{ft}$ BGS on 8-13-96

DEVELOPMENT OBSERVATIONS: Well is an excellent producer, as judged by inability to dry the well up. The well water cleared significantly during development, though not entirely.

OBSERVER SIGNATURE:

DATE:
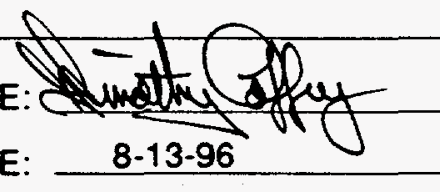
WELL NO GW-835

SITE:

S3 Ponds

APPROX. VOLUME OF DEVELOPMENT WATER: 48 gallons

CALIBRATION OF INSTRUMENTS (check those instruments calibrated to manufacturer's specifications):

$\mathrm{pH}$ meter

Sp. Cond. meter

Organic vapor meter

Beta/gamma meter

Alpha meter
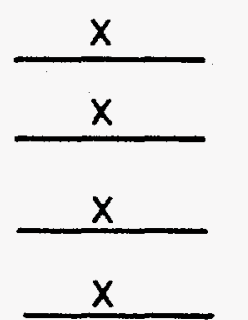

$x$ (model)

(model)

(model)

(model)

(model)
Horiba Model U-7

YSI S-C-T Meter Model 33

Century/Foxboro OVA Model 128 Ludlum Model 3 Survey Meter with G-M Pancake Probe Ludlum Model 12 Count Ratemeter with Scintillation Tube Probe

FIELD SCREENING RESULTS:

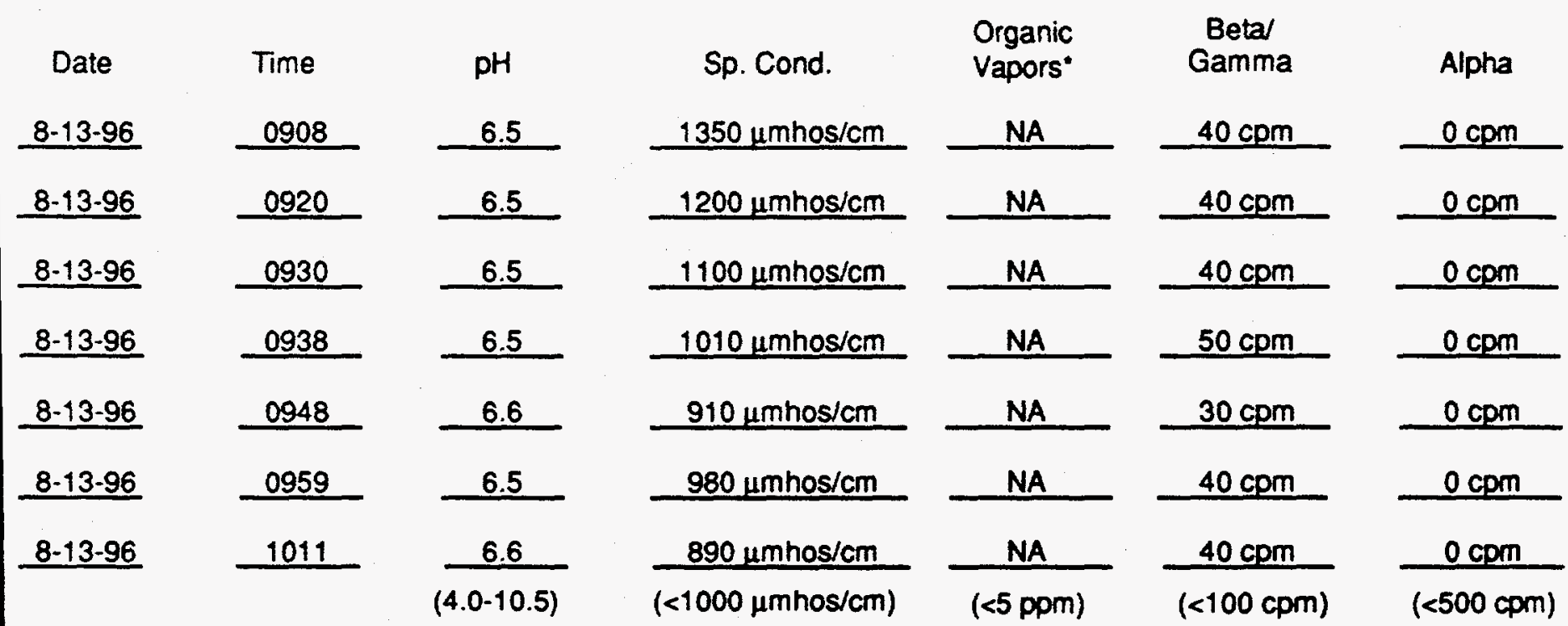

"No headspace analysis, all development water contained.

Weather: Cloudy, breezy

DISPOSITION: Drill-site Disposal

Describe: Development water placed in 330-gal. P-T Tank.
Temp.: Low- to upper-70s ${ }^{\circ} \mathrm{F}$

Containerization

$x$

(Labeled?)Oyn

On-site Geologist (print)

Signature:

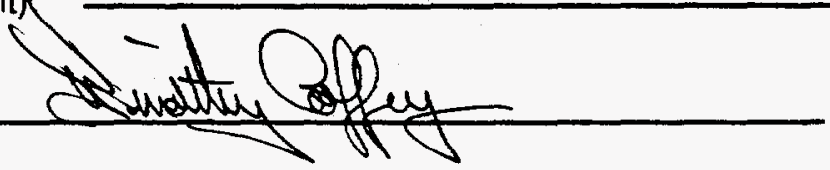

Date: $\quad 8-13-96$ 
Y-12 PLANT GWPP DEVELOPMENT WATER FIELD SCREENING/DISPOSAL SHEET

WELL NO. GW-835

SITE:

S3 Ponds

APPROX. VOLUME OF DEVELOPMENT WATER:

48 gallons

CALIBRATION OF INSTRUMENTS (check those instruments calibrated to manufacturer's specifications):

pH meter

Sp. Cond. meter

Organic vapor meter

Beta/gamma meter

Alpha meter

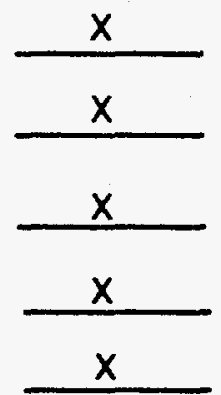

(model)

(model)

(model)

(model)

(model)
Horiba Model U-7

YSI S-C-T Meter Model 33

Century/Foxboro OVA Model 128

Ludlum Model 3 Survey Meter with G-M Pancake Probe

Ludlum Model 12 Count Ratemeter

with Scintillation Tube Probe

FIELD SCREENING RESULTS:

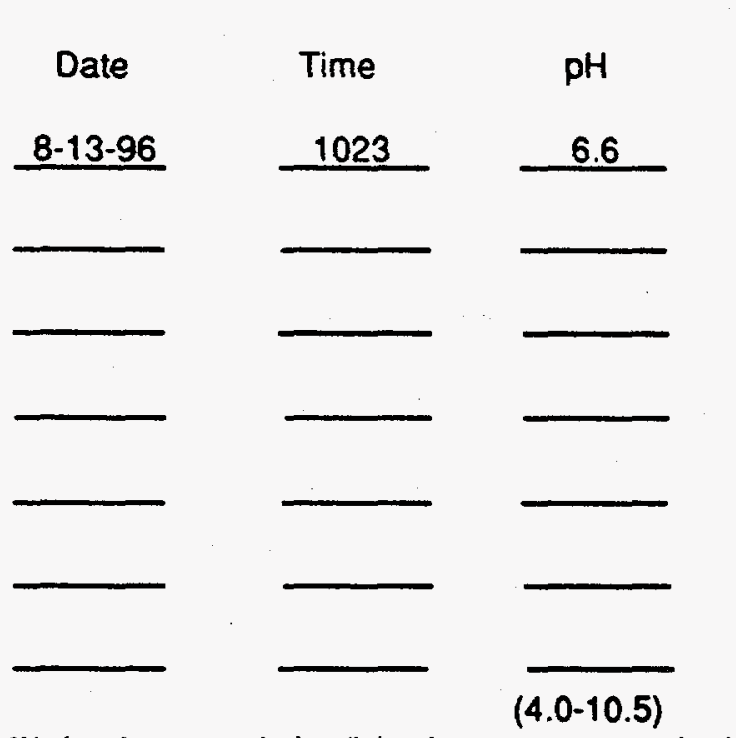

-No headspace analysis, all development water contained.

Sp. Cond.

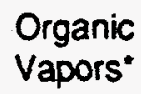

Beta/

Gamma

Alpha

890 umhos/cm

NA

$30 \mathrm{cpm}$

$0 \mathrm{cpm}$

Weather: Cloudy, breezy

DISPOSITION: Drill-site Disposal

Describe: Development water placed in 330-gal. P-T Tank.

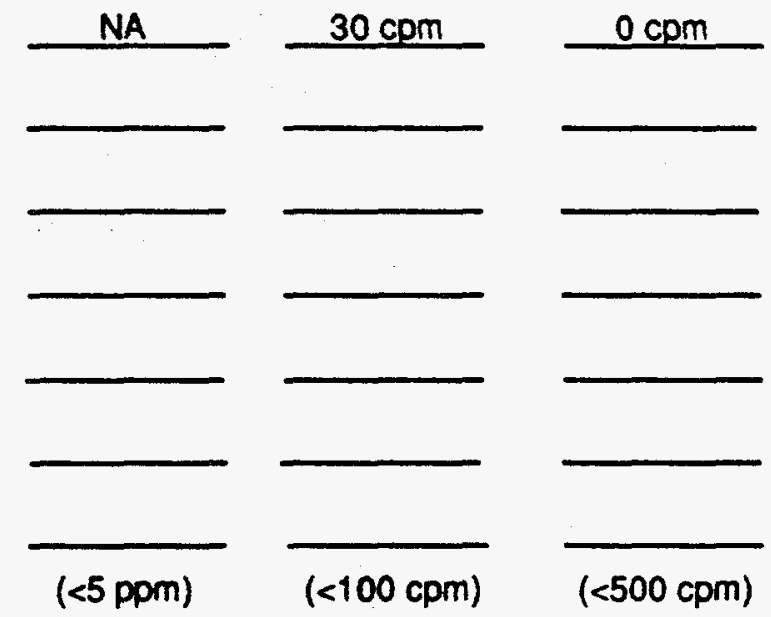

Temp.: Low- to upper-70s"F

Containerization

$x$ (Labeled?)Qn

On-site Geologist (print): Timothy Coffey - SAIC

Signature:

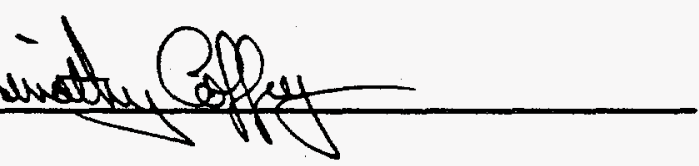

Date: $8-13-96$ 
Y-12 PLANT GROUNDWATER PROTECTION PROGRAM

\section{EQUIPMENT DECONTAMINATION INSPECTION SUMMARY}

LOCATION: S3 Ponds

DECONTAMINATION CREW: G. Shillings
WELL NO. GW-835

INSTALLATION

P\&A

DEVELOPMENT $X$

\begin{tabular}{|l|c|c|c|c|}
\hline \multicolumn{1}{|c|}{ EQUIPMENT } & $\begin{array}{c}\text { DECON } \\
\text { DATE }\end{array}$ & $\begin{array}{c}\text { INSPECTION } \\
\text { DATE }\end{array}$ & $\begin{array}{c}\text { INSPECTION } \\
\text { (PASS/FAlL) }\end{array}$ & $\begin{array}{c}\text { INSPECTOR'S } \\
\text { INITIALS }\end{array}$ \\
\hline $\begin{array}{l}\text { DRILL RIG } \\
\text { (Mast, Chassis, Cables, Carousel, Hoses, Etc.) }\end{array}$ & NA & - & - & - \\
\hline $\begin{array}{l}\text { DRILLING TOOLS (Pipe Wrenches, Hand Tools, Litting } \\
\text { Bells, Clevis, Chains, Etc.) }\end{array}$ & NA & - & - & - \\
\hline $\begin{array}{l}\text { DOWN HOLE TOOLS (Drilling Rods, Stabilizers, } \\
\text { Washover Pipe, Bits, Etc.) }\end{array}$ & NA & - & - & - \\
\hline $\begin{array}{l}\text { WELL CONSTRUCTION MATERIALS (Casing, Screen, } \\
\text { Centralizers, Etc.) }\end{array}$ & NA & - & - & - \\
\hline $\begin{array}{l}\text { WORKOVER RIG } \\
\text { (Mast, Chassis, Cables, Hoses, Etc.) }\end{array}$ & NA & - & - & - \\
\hline DEVELOPMENT TOOLS (Tubing, Bailers, Pumps, Etc.) & $8-9-96$ & $8-13-96$ & Pass & TJC \\
\hline
\end{tabular}

OTHER EQUIPMENT OR RE-INSPECTIONS (SPECIFY)

\begin{tabular}{|l|l|l|l|l|}
\hline & & & & \\
\hline & & & & \\
\hline & & & & \\
\hline & & & & \\
\hline COMMENTS: & & & & \\
\hline & & & & \\
\hline & & & & \\
\hline
\end{tabular}




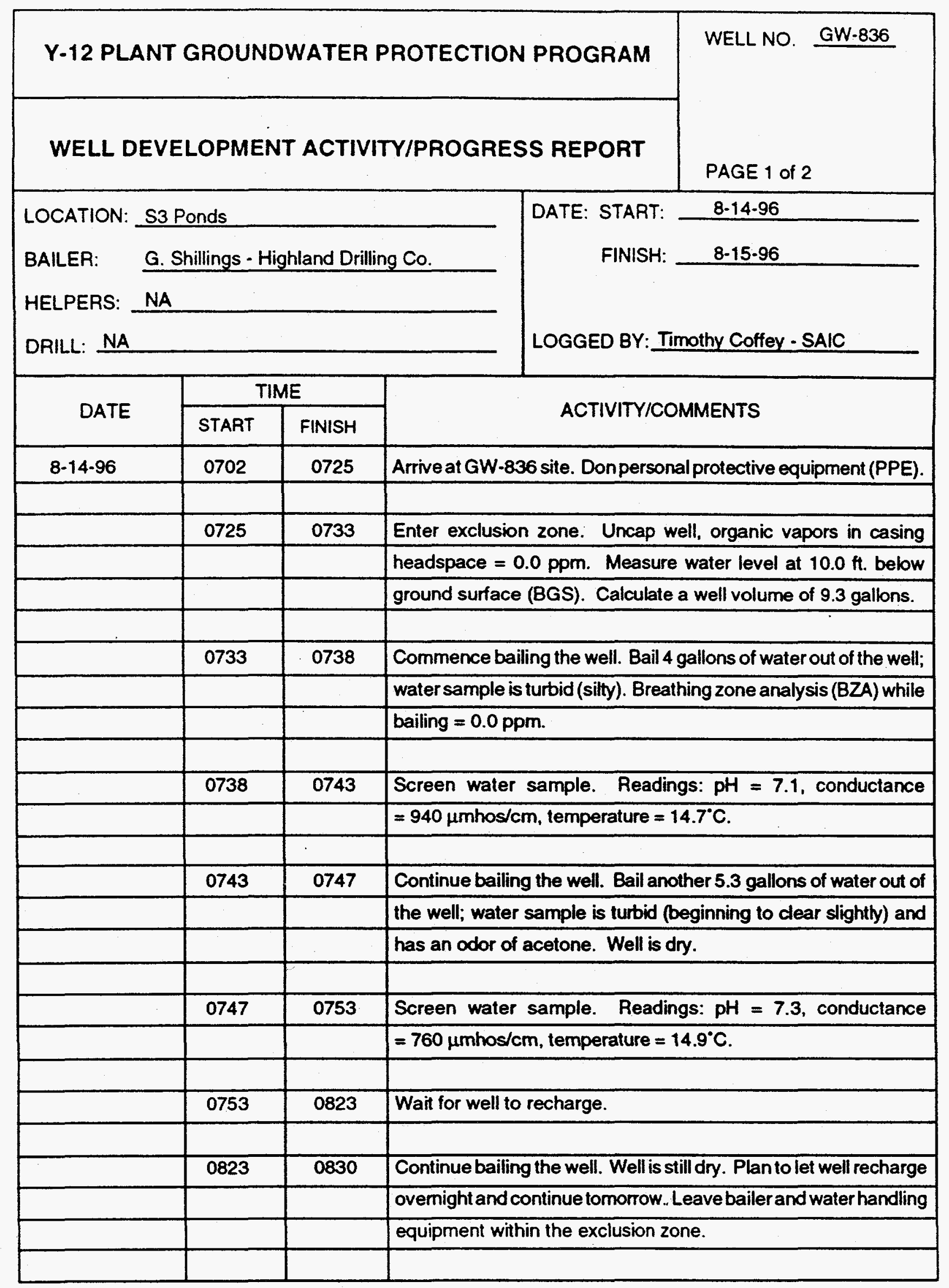




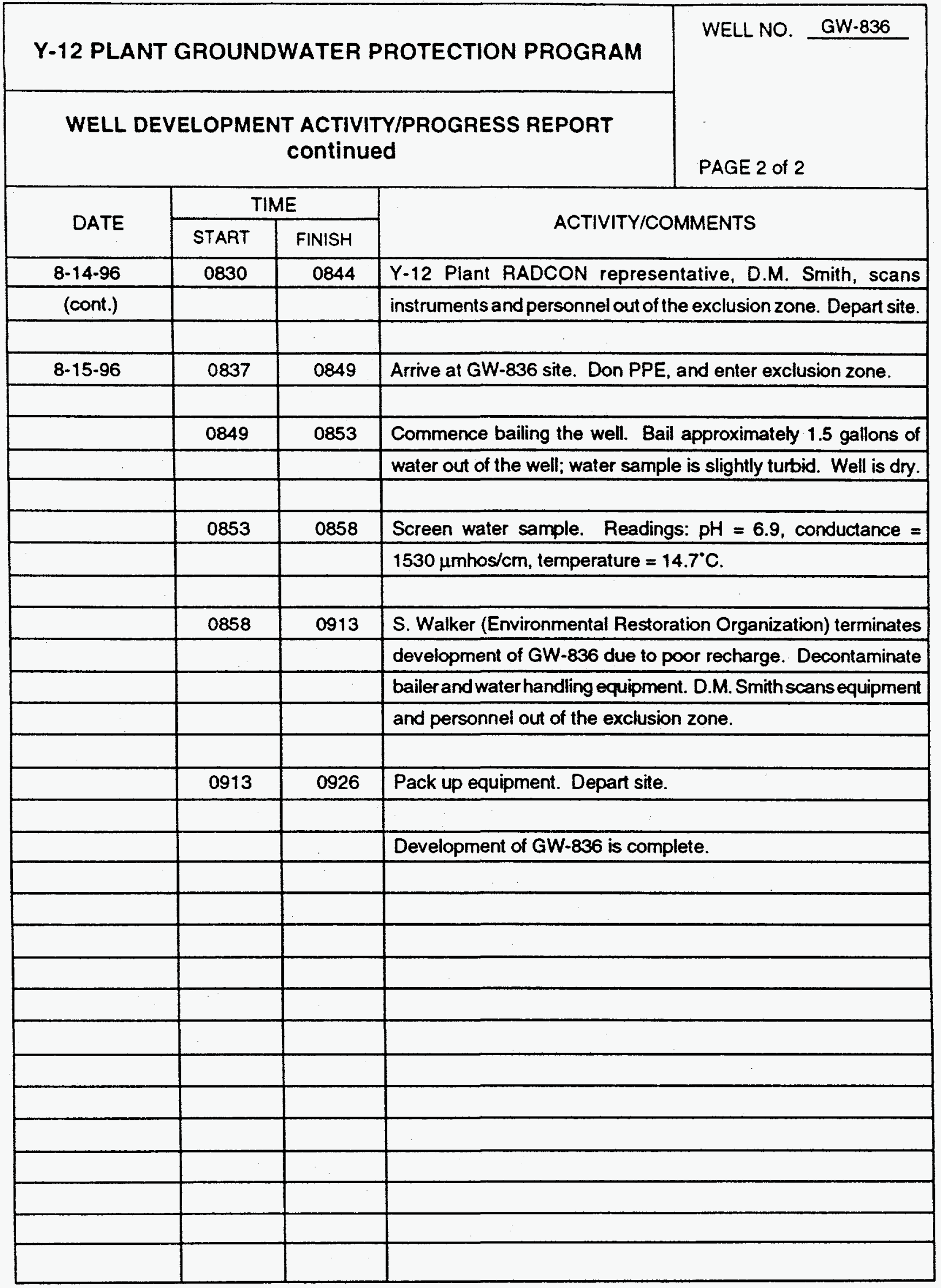




\section{Y-12 PLANT GROUNDWATER PROTECTION PROGRAM}

\section{MONITORING WELL DEVELOPMENT PROGRESS}

WELL NO. GW-836

LOCATION: S3 Ponds

ONE WELL VOLUME: $\quad 9.3$

GALLONS

WELL INSTALLATION

COMPLETION DATE:

7.16-96

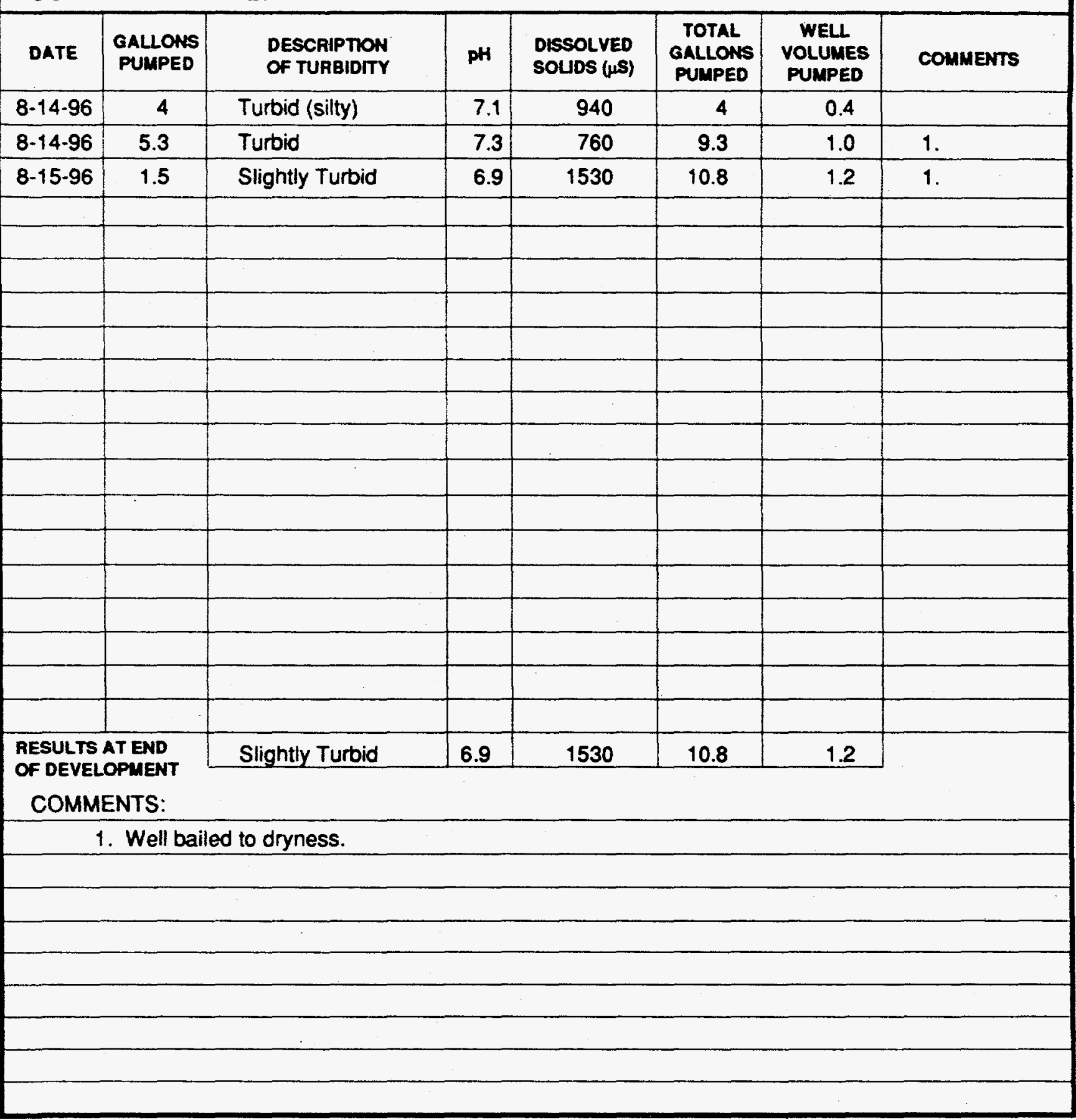




\section{Y-12 PLANT GROUNDWATER PROTECTION PROGRAM}

\section{MONITORING WELL DEVELOPMENT SUMMARY}

WELL NO. GW-836

METHOD OF DEVELOPMENT: 4-liter bailer

DEVELOPMENT DATE:

DEVELOPMENT OBSERVED BY: Timothy Coffey - SAIC

ONE WELL VOLUME:

9.3 GALLONS

TOTAL GALLONS PUMPED:

10.8 TOTAL WELL VOLUMES PUMPED:

1.2

INITIAL pH: 7.1 FINAL pH: 6.9

INITIAL SPECIFIC CONDUCTANCE: $940 \mu \mathrm{mhos} / \mathrm{cm}$

FINAL: $1530 \mu \mathrm{mhos} / \mathrm{cm}$

DESCRIPTION OF INITIAL TURBIDITY:

DESCRIPTION OF FINAL TURBIDITY:

FINAL MEASURED TURBIDITY:

WELL APPROVED BY:

START: $8-14-96$

FINISH: $\quad$ 8-15-96 
WELL NO. GW-836

SITE: S3 Ponds

APPROX. VOLUME OF DEVELOPMENT WATER: 10.8 gallons

CALIBRATION OF INSTRUMENTS (check those instruments calibrated to manufacturer's specifications):

pH meter

Sp. Cond. meter

Organic vapor meter

Beta/gamma meter

Alpha meter

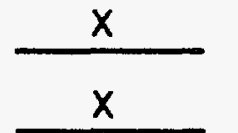

$x$

$\frac{\frac{x}{x}}{x}$

(model)

(model)

(model)

(model)

(model)
Horiba Model U-7

YSI S-C-T Meter Model 33

Century/Foxboro OVA Model 128

Ludlum Model 3 Survey Meter with

G.M Pancake Probe

Ludlum Model 12 Count Ratemeter

with Scintillation Tube Probe

FIELD SCREENING RESULTS:

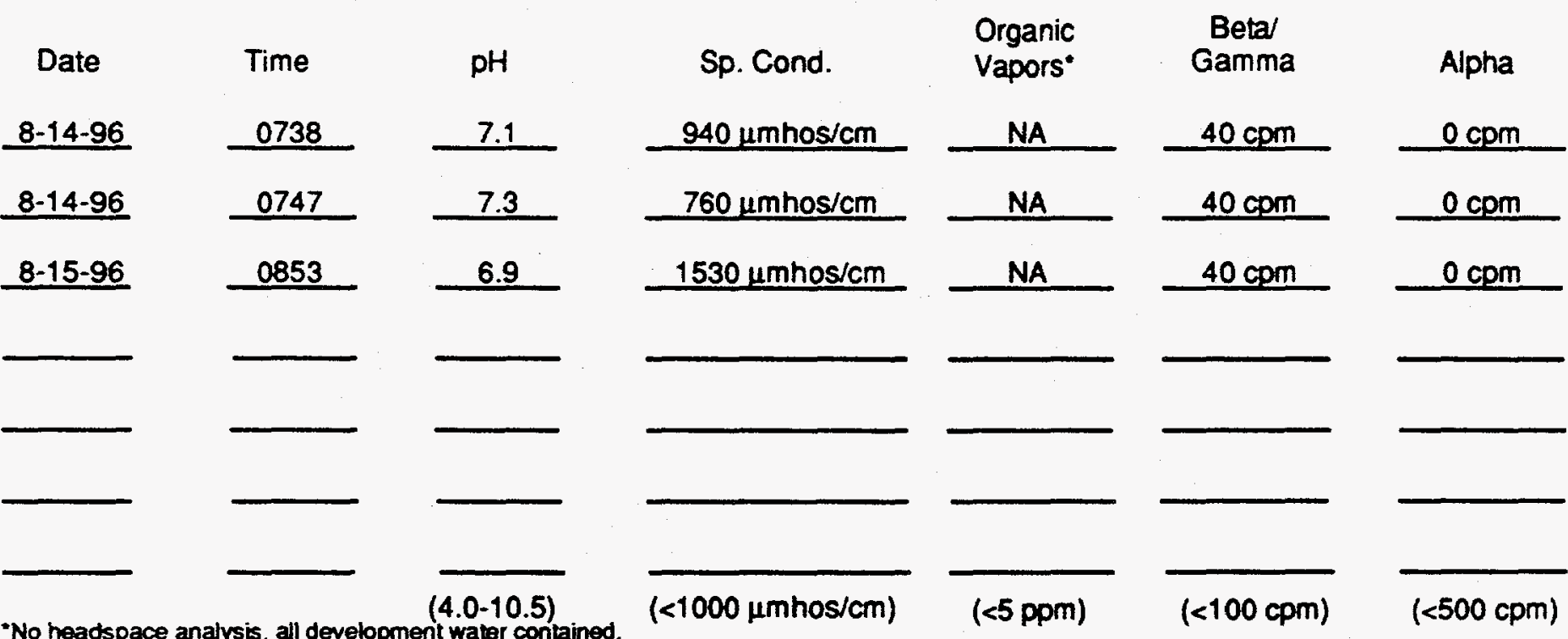

-No headspace analysis, all development water contained.

8-14-96: Early a.m. fog, then clear.

Weather: 8-15-96: Early a.m. fog, then clear.

8-14-96: Low- to upper-60s ${ }^{\circ} \mathrm{F}$

Temp.: 8-15-96: Low- to upper-60s ${ }^{\circ} \mathrm{F}$

DISPOSITION: Drill-site Disposal

Containerization $x$

Describe: Development water placed in 330-gal. P-T Tank.

(Labeled?)ogn

On-site Geologist (print):

Timothy Coffey - SAIC

Signature:

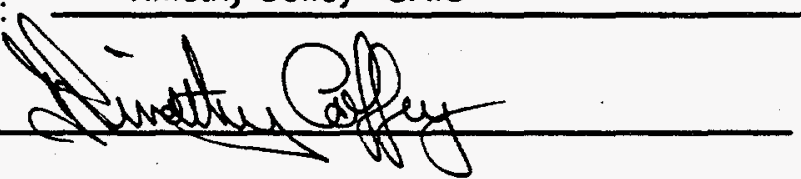

Date: $\quad 8-13-96$ 
Y-12 PLANT GROUNDWATER PROTECTION PROGRAM

\section{EQUIPMENT DECONTAMINATION INSPECTION SUMMARY}

LOCATION:

S3 Ponds

DECONTAMINATION CREW: G. Shillings
WELL NO. GW.836

INSTALLATION

P\&A

DEVELOPMENT

DATE: START: $8-14-96$

FINISH: 8-15.96

\begin{tabular}{|l|c|c|c|c|}
\hline \multicolumn{1}{|c|}{ EQUIPMENT } & $\begin{array}{c}\text { DECON } \\
\text { DATE }\end{array}$ & $\begin{array}{c}\text { INSPECTION } \\
\text { DATE }\end{array}$ & $\begin{array}{c}\text { INSPECTION } \\
\text { (PASS/FAIL) }\end{array}$ & $\begin{array}{c}\text { INSPECTOR'S } \\
\text { INITIALS }\end{array}$ \\
\hline $\begin{array}{l}\text { DRILL RIG } \\
\text { (Mast, Chassis, Cables, Carousel, Hoses, EIc.) }\end{array}$ & NA & - & - & - \\
\hline $\begin{array}{l}\text { DRILLING TOOLS (Pipe Wrenches, Hand Tools, Lifting } \\
\text { Bells, Clevis, Chains, Etc.) }\end{array}$ & NA & - & - & - \\
\hline $\begin{array}{l}\text { DOWN HOLE TOOLS (Drilling Rods, Stabilizers, } \\
\text { Washover Pipe, Bits, Etc.) }\end{array}$ & NA & - & - & - \\
\hline $\begin{array}{l}\text { WELL CONSTRUCTION MATERIALS (Casing, Screen, } \\
\text { Centralizers, Etc.) }\end{array}$ & NA & - & - & - \\
\hline $\begin{array}{l}\text { WORKOVER RIG } \\
\text { (Mast, Chassis, Cables, Hoses, Etc.) }\end{array}$ & NA & - & - & - \\
\hline DEVELOPMENT TOOLS (Tubing, Bailers, Pumps, Etc.) & $8-13-96$ & $8-13-96$ & Pass & TJC \\
\hline
\end{tabular}

\begin{tabular}{|l|l|l|l|l|}
\hline OTHER EQUIPMENT OR RE-INSPECTIONS (SPECIFY & & & & \\
\hline & & & & \\
\hline & & & & \\
\hline & & & & \\
\hline & & & & \\
\hline COMMENTS: & & & & \\
\hline & & & \\
\hline
\end{tabular}




\section{Y-12 PLANT GROUNDWATER PROTECTION PROGRAM}

WELL NO. GW-837

\section{WELL DEVELOPMENT ACTIVITYIPROGRESS REPORT}

PAGE 1 of 4

LOCATION: S3 Ponds

BAILER: J. Gallaher - Highland Drilling Co.

HELPERS: NA

DRILL: NA
DATE: START: $\quad 8-16-96$

FINISH:

8-19-96

LOGGED BY: Timothy Coffey - SAIC

\section{ACTIVITY/COMMENTS}

Arrive at GW-837 site. Waiting for water containment tank to be brought to site.

Don personal protective equipment (PPE).

Enter exclusion zone. Uncap well, organic vapors: in casing headspace $=12 \mathrm{ppm}(\max )$, in breathing zone $=0.0 \mathrm{ppm}$.

Measure water level at $8.2 \mathrm{ft}$ below ground surface (BGS).

Calculate a well volume of 12.9 gallons.

Commence bailing the well. Bail 4 gallons of water out of the well; water sample is turbid. Water sample also has an indistinguishable chemical odor.

Screen water sample. Readings: $\mathrm{pH}=5.7$, conductance $=8900 \mu \mathrm{mhos} / \mathrm{cm}$, temperature $=18.1^{\circ} \mathrm{C}$.

Continue bailing the well. Bail another 7 gallons of water out of the well; water sample is turbid, but appears to be clearing slightly. Water still has a chemical odor.

Screen water sample. Readings: $\mathrm{pH}=5.6$, conductance $=8900 \mu \mathrm{mhos} / \mathrm{cm}$, temperature $=18.9^{\circ} \mathrm{C}$. 


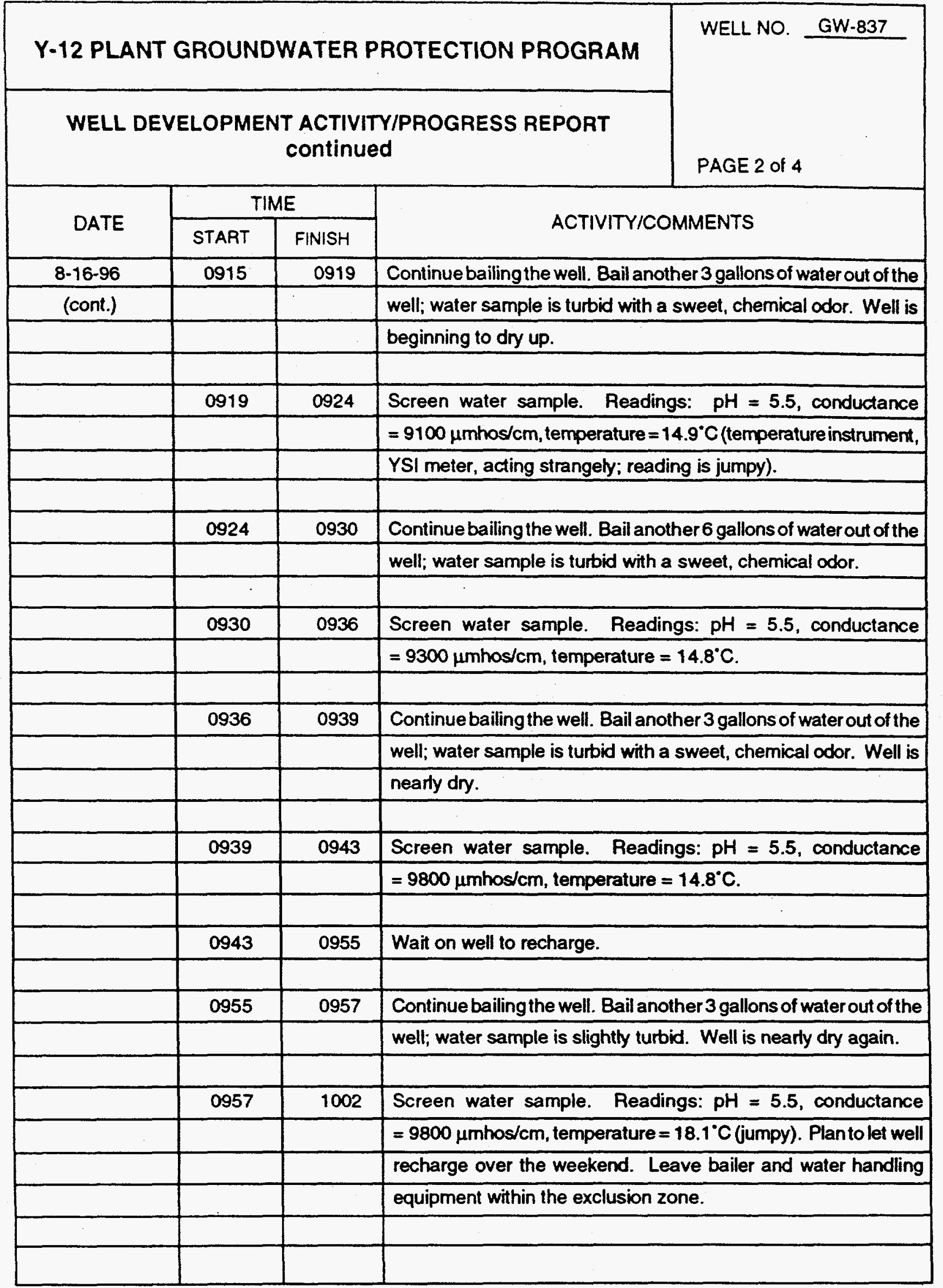




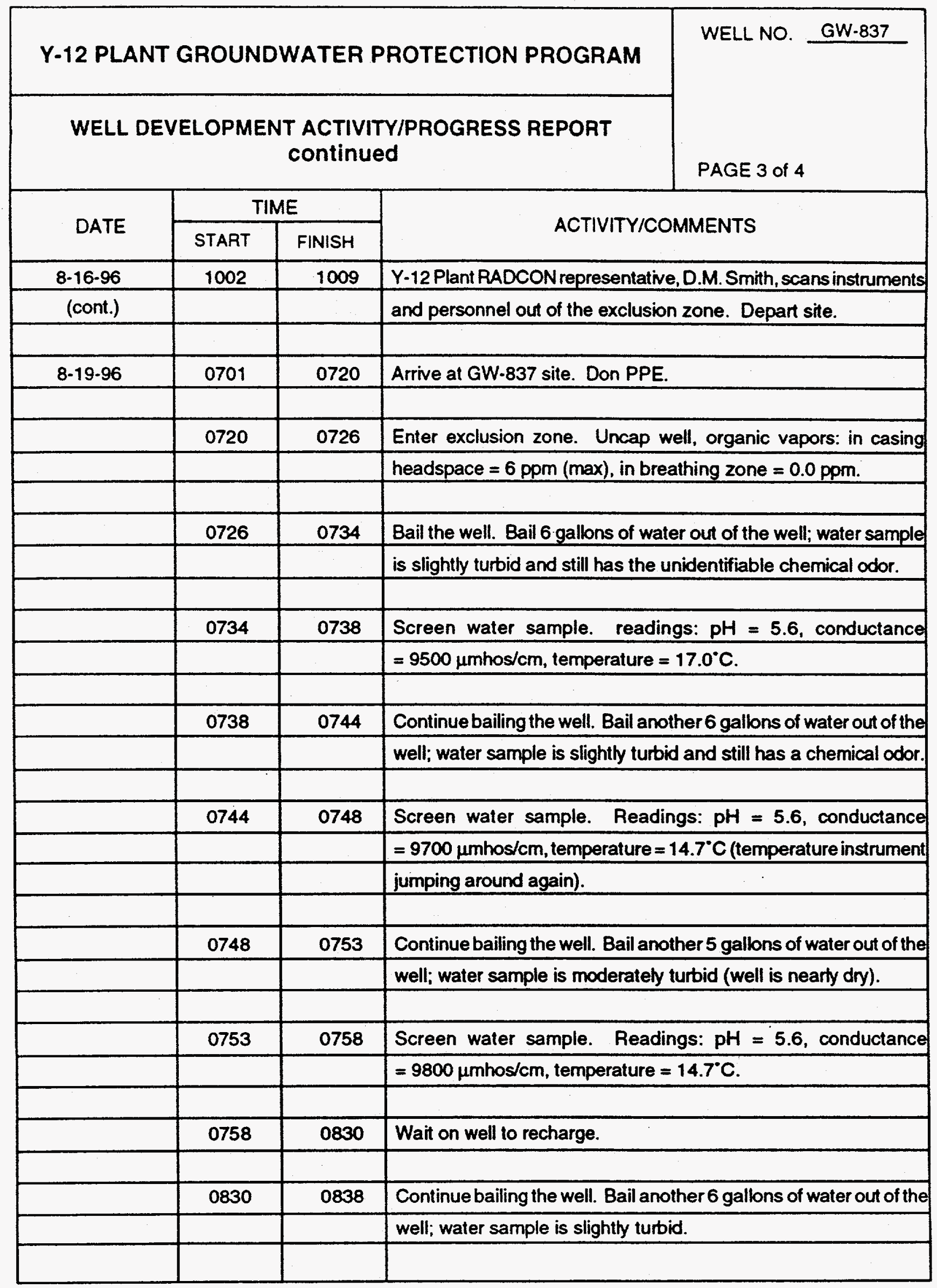


Y-12 PLANT GROUNDWATER PROTECTION PROGRAM

WELL NO. GW-837

WELL DEVELOPMENT ACTIVITY/PROGRESS REPORT continued

PAGE 4 of 4

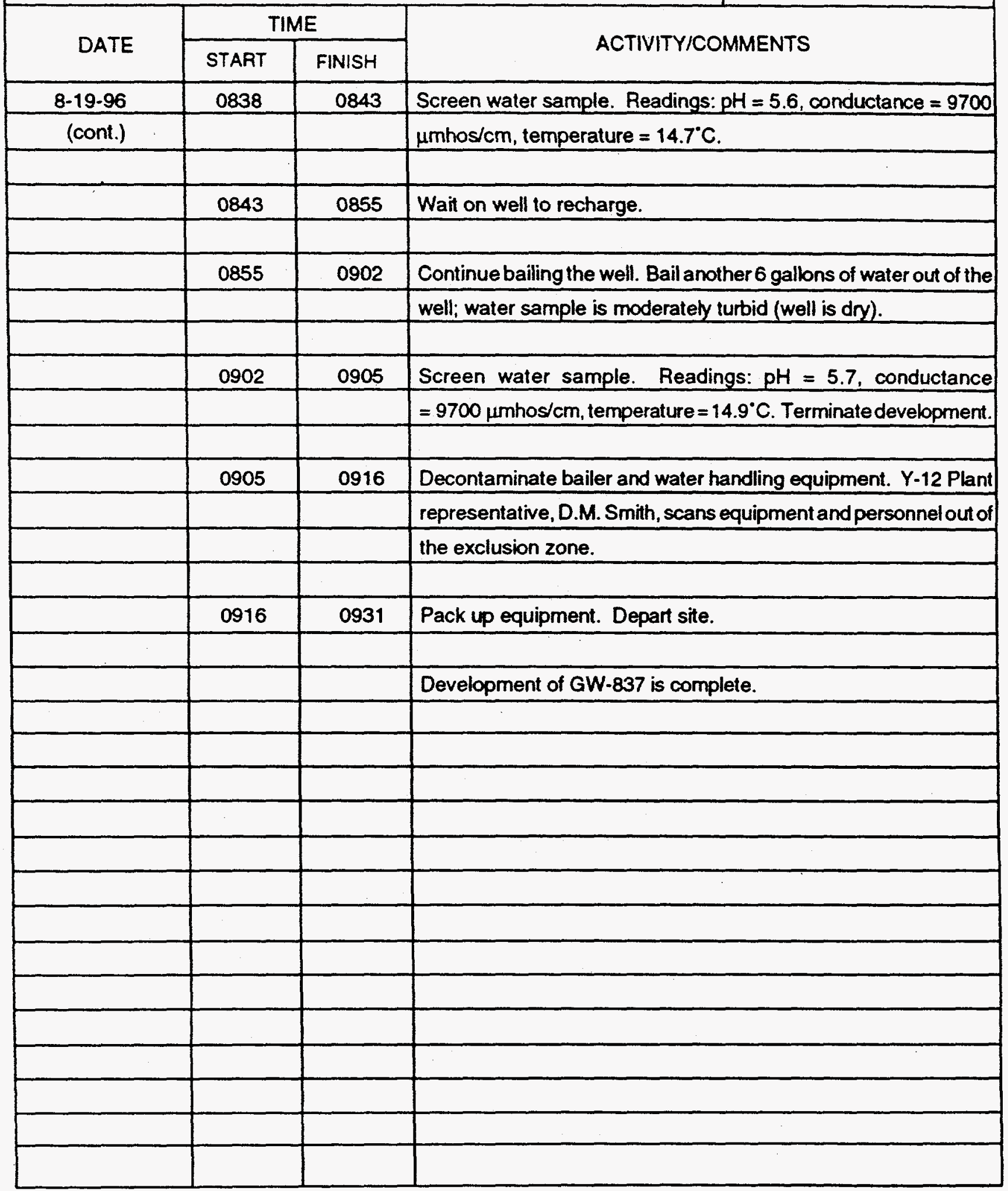




\section{Y-12 PLANT GROUNDWATER PROTECTION PROGRAM}

\section{MONITORING WELL DEVELOPMENT PROGRESS}

WELL NO. GW-837

LOCATION: S3 PONdS ONE WELL VOLUME: 12.9 GALLONS

WELL INSTALLATION

COMPLETION DATE: 7-17-96

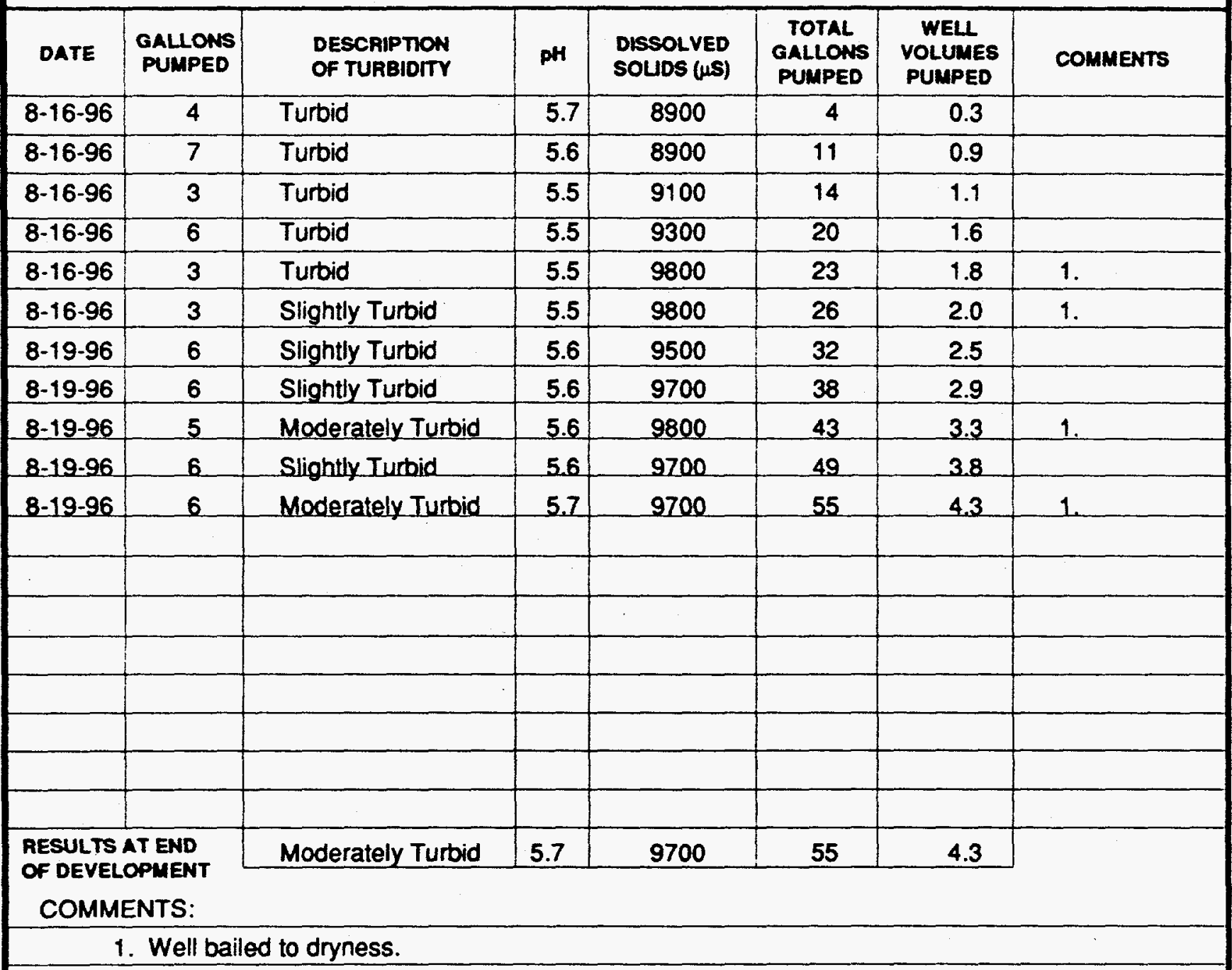




\section{Y-12 PLANT GROUNDWATER PROTECTION PROGRAM}

WELL NO. GW-837

\section{MONITORING WELL DEVELOPMENT SUMMARY}

METHOD OF OEVELOPMENT: 4 -liter bailer

DEVELOPMENT OBSERVED BY: Timothy Coffey - SAIC

DEVELOPMENT DATE:

ONE WELL VOLUME: $12.9 \quad$ GALLONS

TOTAL GALLONS PUMPED: $\quad 55$ TOTAL WELL VOLUMES PUMPED: $\quad 4.3$
INITIAL pH:
5.7
FINAL pH:
5.7

INITIAL SPECIFIC CONDUCTANCE:

$8900 \mu \mathrm{mhos} / \mathrm{cm}$

FINAL: $9700 \mu \mathrm{mhos} / \mathrm{cm}$

DESCRIPTION OF INITIAL TURBIDITY:

DESCRIPTION OF FINAL TURBIDITY:

FINAL MEASURED TURBIDITY:

Turbid

START: $\quad 8-16-96$

FINISH: $\quad$ 8-19-96

WELL APPROVED BY:

Moderately Turbid

Not Analyzed

W.K. Jago - HSEA

ODOR OF WATER: Unidentifiable, sweetpungent chemical odor
WATER DISCHARGED TO:
$\square$ GROUND SURFACE
$\square$ TANK TRUCK
$\square$ STORM SEWERS
$\triangle$ STORAGE TANKS
$\square$ DRUMS
$\square$ OTHER

INITIAL PRE-DEVELOPMENT

WATER DEPTH:

8.2 ft BGS on 8-16-96

DEVELOPMENT OBSERVATIONS: Well is a moderate to good producer. Well easily gave up more than 1 well volume during development sessions, and appears to recharge fairly quickly. Overall, the water cleared fairly significantly, but samples obtained prior to drying up were slightly more turbid (from sediment accumulation still at bottom of well).

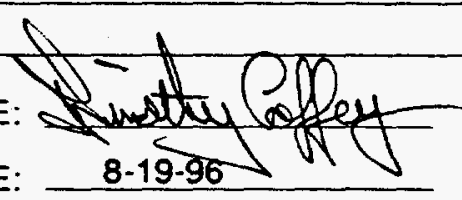


WELL NO. GW-837

SITE: S3 Ponds

APPROX. VOLUME OF DEVELOPMENT WATER: 55 gallons

CALIBRATION OF INSTRUMENTS (check those instruments calibrated to manufacturer's specifications):

pH meter

Sp. Cond. meter

Organic vapor meter

Beta/gamma meter

Alpha meter

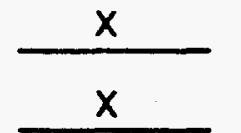

$\frac{\frac{x}{x}}{\frac{x}{x}}$

(model)

(model)

(model)

(model)

(model)
Horiba Model U-7

YSI S-C-T Meter Model 33

Century/Foxboro OVA Model 128

Ludlum Model 3 Survey Meter with G-M Pancake Probe

Ludlum Model 12 Count Ratemeter

with Scintillation Tube Probe

FIELD SCREENING RESULTS:

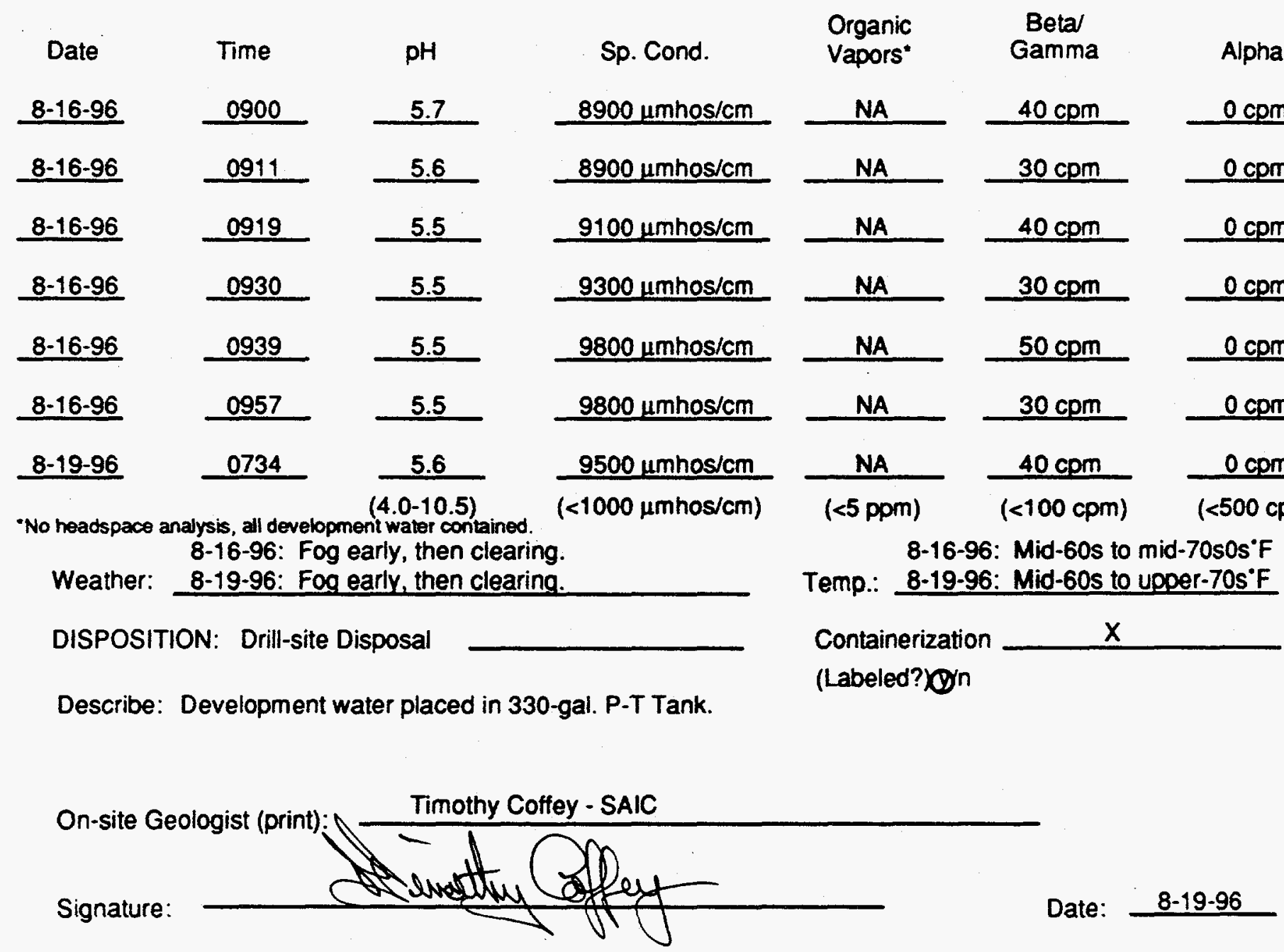




\section{Y.12 PLANT GWPP DEVELOPMENT WATER FIELD SCREENING/DISPOSAL SHEET}

WELL NO. GW-837 SITE:

APPROX. VOLUME OF DEVELOPMENT WATER:

CALIBRATION OF INSTRUMENTS (check those instruments calibrated to manufacturer's specifications):
$\mathrm{pH}$ meter

Sp. Cond. meter

Organic vapor meter

Beta/gamma meter

Alpha meter
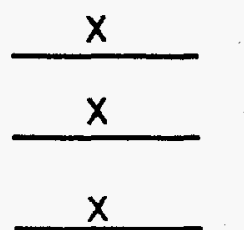

$\frac{x}{x}$

$\frac{x}{x}$

(model)

(model)

(model)

(model)

(model)
S3 Ponds

55 gallons
FIELD SCREENING RESULTS:

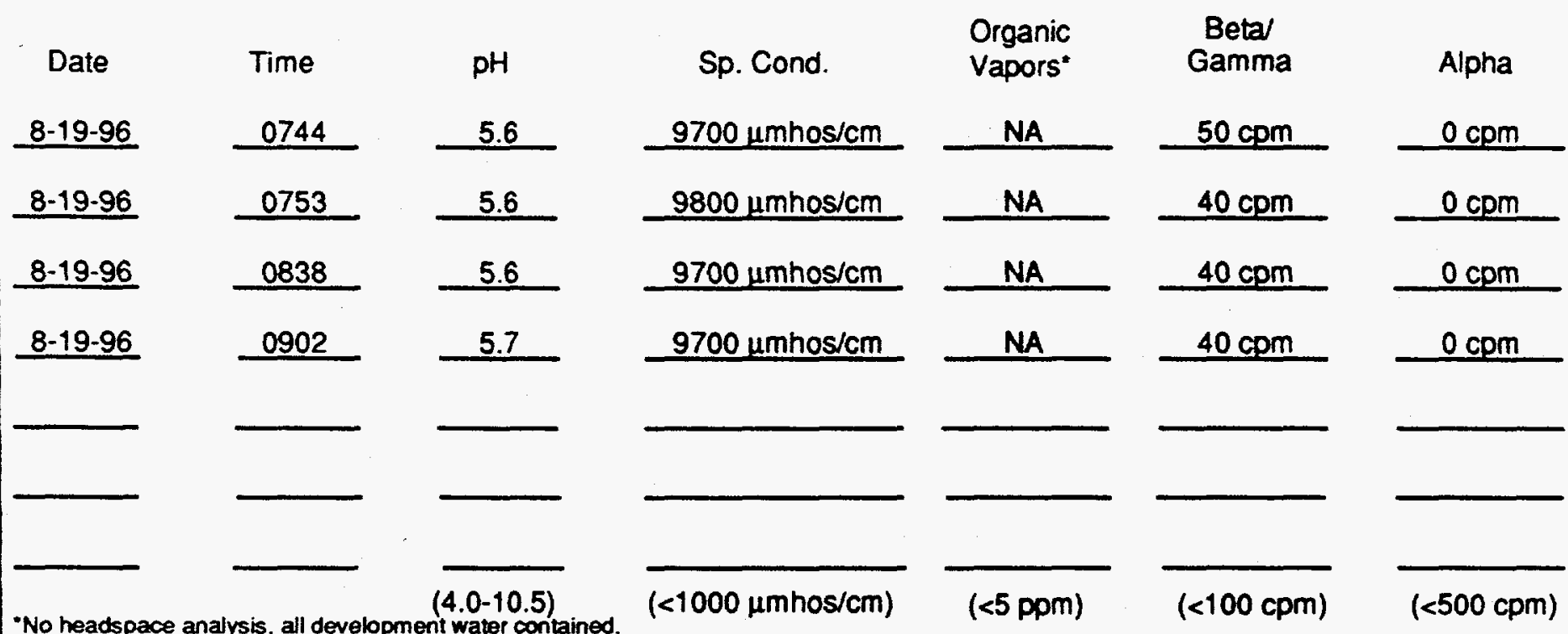

"No headspace analysis, all development water contained.

$$
\text { 8-16-96: Fog early, then clearing. }
$$

Weather: 8-19-96: Fog early, then clearing.

8-16-96: Mid-60s to mid-70s0s ${ }^{\circ} \mathrm{F}$ Temp.: 8-19-96: Mid-60s to upper-70s ${ }^{\circ} \mathrm{F}$

Containerization $x$

DISPOSITION: Drill-site Disposal

(Labeled?)ogn

Century/Foxboro OVA Model 128

Ludlum Model 3 Survey Meter with

G-M Pancake Probe

Ludlum Model 12 Count Ratemeter

with Scintillation Tube Probe

Describe: Development water placed in 330-gal. P-T Tank.

On-site Geologist (print):

Timothy Coffey - SAIC

Signature:

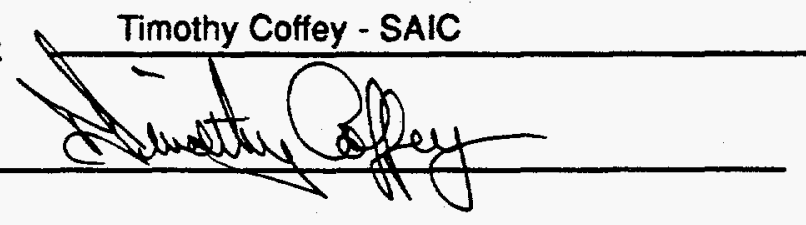

Date: 
Y-12 PLANT GROUNDWATER PROTECTION PROGRAM

\section{EQUIPMENT DECONTAMINATION INSPECTION SUMMARY}

LOCATION: S3 Ponds

DECONTAMINATION CREW: J. Gallaher
WELL NO. GW-837

INSTALLATION

P\&A

DEVELOPMENT $X$

\begin{tabular}{|l|c|c|c|c|}
\hline \multicolumn{1}{|c|}{ EQUIPMENT } & $\begin{array}{c}\text { DECON } \\
\text { DATE }\end{array}$ & $\begin{array}{c}\text { INSPECTION } \\
\text { DATE }\end{array}$ & $\begin{array}{c}\text { INSPECTION } \\
\text { (PASS/FAIL) }\end{array}$ & $\begin{array}{c}\text { INSPECTOR'S } \\
\text { INITIALS }\end{array}$ \\
\hline $\begin{array}{l}\text { ORILL RIG } \\
\text { (Mast, Chassis, Cables, Carousel, Hoses, Etc.) }\end{array}$ & NA & - & - & - \\
\hline $\begin{array}{l}\text { DRILLING TOOLS (Pipe Wrenches, Hand Tools, Litting } \\
\text { Bells, Clevis, Chains, Etc.) }\end{array}$ & NA & - & - & - \\
\hline $\begin{array}{l}\text { DOWN HOLE TOOLS (Drilling Rods, Stabilizers, } \\
\text { Washover Pipe, Bits, Etc.) }\end{array}$ & NA & - & - & - \\
\hline $\begin{array}{l}\text { WELL CONSTRUCTION MATERIALS (Casing, Screen, } \\
\text { Centralizers, Etc.) }\end{array}$ & NA & - & - & - \\
\hline $\begin{array}{l}\text { WORKOVER RIG } \\
\text { (Mast, Chassis, Cables, Hoses, Etc.) }\end{array}$ & NA & - & - & - \\
\hline DEVELOPMENT TOOLS (Tubing, Bailers, Pumps, Etc.) & $8-16-96$ & $8-16-96$ & Pass & TJC \\
\hline
\end{tabular}

OTHER EQUIPMENT OR RE-INSPECTIONS (SPECIFY)

COMMENTS: 


\section{APPENDIX B}

AQUIFER PUMPING TEST RESULTS 

This appendix presents the results of the aquifer pumping test performed on November 12 , 1996 , in pumped piezometer (GW-835) and nine nearby observation piezometers (GW-836 and TPB-06, -07, -15, -16, -18, -26, -29, and -30) (Fig. B.1). A constant flow pumping test was attempted to characterize the unconsolidated aquifer and estimate the pumping flow rates the piezometer could sustain with time.

A pumping pre-test was performed in GW-835 on November 4, 1996, to determine the recharge rate of the aquifer and confirm the potential for the piezometer to serve as the pumped piezometer for the upcoming aquifer test. A bladder pump was installed and used to pump groundwater from the piezometer to determine the recharge rate of the aquifer. A recharge rate of $2.5 \mathrm{~L} / \mathrm{min}$ was measured while a constant water level elevation was observed in the piezometer. Attempts to increase the discharge rate above $2.5 \mathrm{~L} / \mathrm{min}$ were unsuccessful due to limitations of the bladder (pneumatic) pump and the water column above the intake of the pump $(\sim 6.5 \mathrm{ft})$.

These results confirmed that GW-835 could yield a sufficient volume of groundwater to allow the performance of the aquifer pumping test. It also determined that a rate of $2.5 \mathrm{~L} / \mathrm{min}$ would be the minimum rate that the piezometer would be pumped for the duration of the test.

Pumping pre-tests were also performed in GW-834 and GW-837. Recharge rates were 60 $\mathrm{mL} / \mathrm{min}$ and $50 \mathrm{~mL} / \mathrm{min}$, respectively. Based on the results, aquifer pump tests were not proposed at these locations. GW-836 was not pre-tested due to extremely slow recharge rates observed during the attempted development of the piezometer (Appendix A).

Implementation. A pneumatic purge pump with a discharge capacity of up to $15 \mathrm{~L} / \mathrm{min}$ was installed in GW-835 to be used during the aquifer pumping test. Table B.1 presents the schedule developed for recording of the water levels in the pumped and observations piezometers.

Table B.1. Measurement frequency in piezometers

\begin{tabular}{lcclcc}
\hline & $\begin{array}{c}\text { Time period } \\
(\mathrm{min})\end{array}$ & $\begin{array}{c}\text { Measurement } \\
\text { frequency }\end{array}$ & & $\begin{array}{c}\text { Time period } \\
(\mathrm{min})\end{array}$ & $\begin{array}{c}\text { Measurement } \\
\text { frequency }\end{array}$ \\
\hline \multirow{3}{*}{ GW-835: } & $0-5$ & $15 \mathrm{sec}$ & pbservation & & \\
(pumped & $5-10$ & $30 \mathrm{sec}$ & $(\mathrm{GW}-836$, & $60-120$ & $5 \mathrm{~min}$ \\
piezometer) & $10-20$ & $1 \mathrm{~min}$ & TPB-06, -07, & $120-240$ & $10 \mathrm{~min}$ \\
& $20-60$ & $5 \mathrm{~min}$ & $-15,-16,-18$, & $240-360$ & $30 \mathrm{~min}$ \\
& $60-300$ & $30 \mathrm{~min}$ & $-26,-29$, and & $360-$ termin. & 60 min \\
& & & $-30)$ & & \\
\hline
\end{tabular}

The test was initiated at 0850 on November 12,1996 . Prior to beginning the test, water levels were measured in the pumped and observation piezometers to determine pre-test water table level. The elevation 


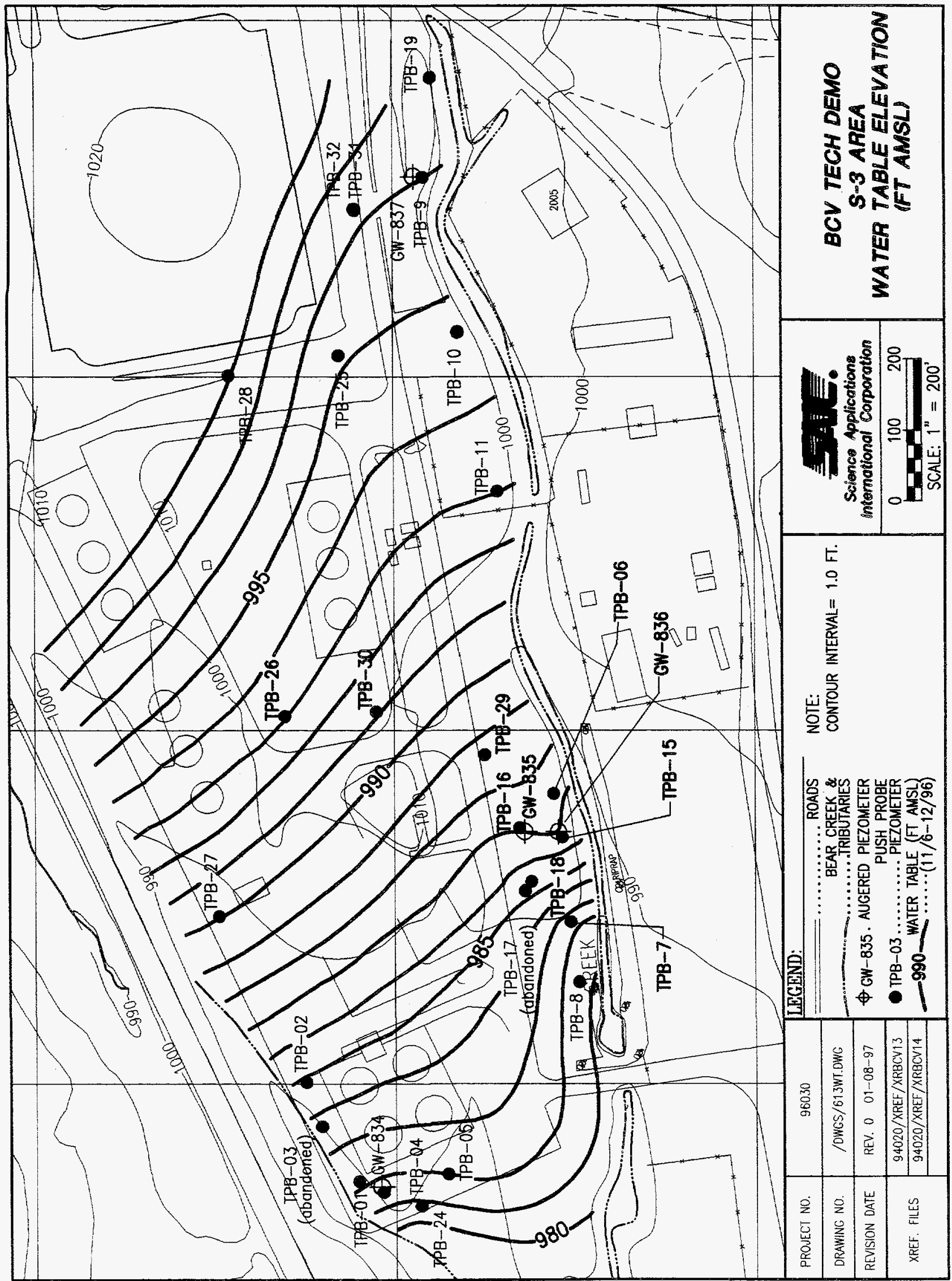

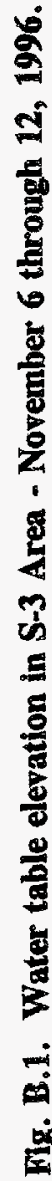




\section{B-5}

of the water table prior to the test is shown in Fig. B.1. Depths to water in the piezometers are a composite of water levels measured from November 6 through 12, 1996.

Results. The water level responses during the aquifer pumping test are shown in Fig. B.2. Little response to the pumping of GW-835 was noted in the observation piezometers. Much of the fluctuation in water levels could most likely be attributed to normal daily variation in the water table. Slight drawdown, though, was observed in TPB-18, but as the test progressed the piezometer appeared to recharge. Water levels in piezometers GW-836 and TPB-07, $-26,-29$, and -30 rose in elevation as the test progressed.

Based on the lack of response in the observation piezometers (especially in TPB-16, $\sim 8 \mathrm{ft}$ from GW-835), pumping rate was increased in an attempt to observe a constant rate of drawdown in the pumped piezometer. A rate $\sim 6 \mathrm{~L} / \mathrm{min}$ was established and maintained over the greater part of the test period. This rate appeared to be the maximum possible using the installed purge pump. The water level in the pumped well (GW-835) stabilized and remained relatively constant at this flow rate.

At 1725 , the pump began to cycle erratically. The test was terminated at this time based on continued lack of response in the observation piezometers. Within $5 \mathrm{~min}$ of turning the pump off in GW-835, water level returned to within $0.07 \mathrm{ft}$ of the pre-test level. Total drawdown in GW-835 during the test was $\sim 4$ $\mathrm{ft}$ from pre-test water level.

Groundwater grab samples were collected at the beginning, middle, and near end of the test period. These samples were analyzed for radiochemistry (gross alpha/beta and fluorimetric uranium), nitrate (as nitrogen), and TDS by the Y-12 ASO. Analytical results are presented in Table B.2.

Table B.2. Aquifer test period groundwater analytical results from GW-835

\begin{tabular}{lccc}
\hline \multicolumn{1}{c}{ Sample no. } & BPA2D1 & BPA2D2 & BPA2D3 \\
Collection date & $11 / 12 / 96$ & $11 / 12 / 96$ & $11 / 12 / 96$ \\
Collection time & 0932 & 1247 & 1430 \\
\hline & Analysis & & \\
Alpha activity, pC/L & 550 & 490 & 500 \\
Beta activity, pC/L & 260 & 260 & 230 \\
Uranium (fluorimetric), ppm & 1.3 & 1.2 & 1.2 \\
Nitrite as Nitrogen, mg/L & 25 & 24 & 24 \\
Total dissolved solids, mg/L & 690 & 670 & 680 \\
\hline
\end{tabular}




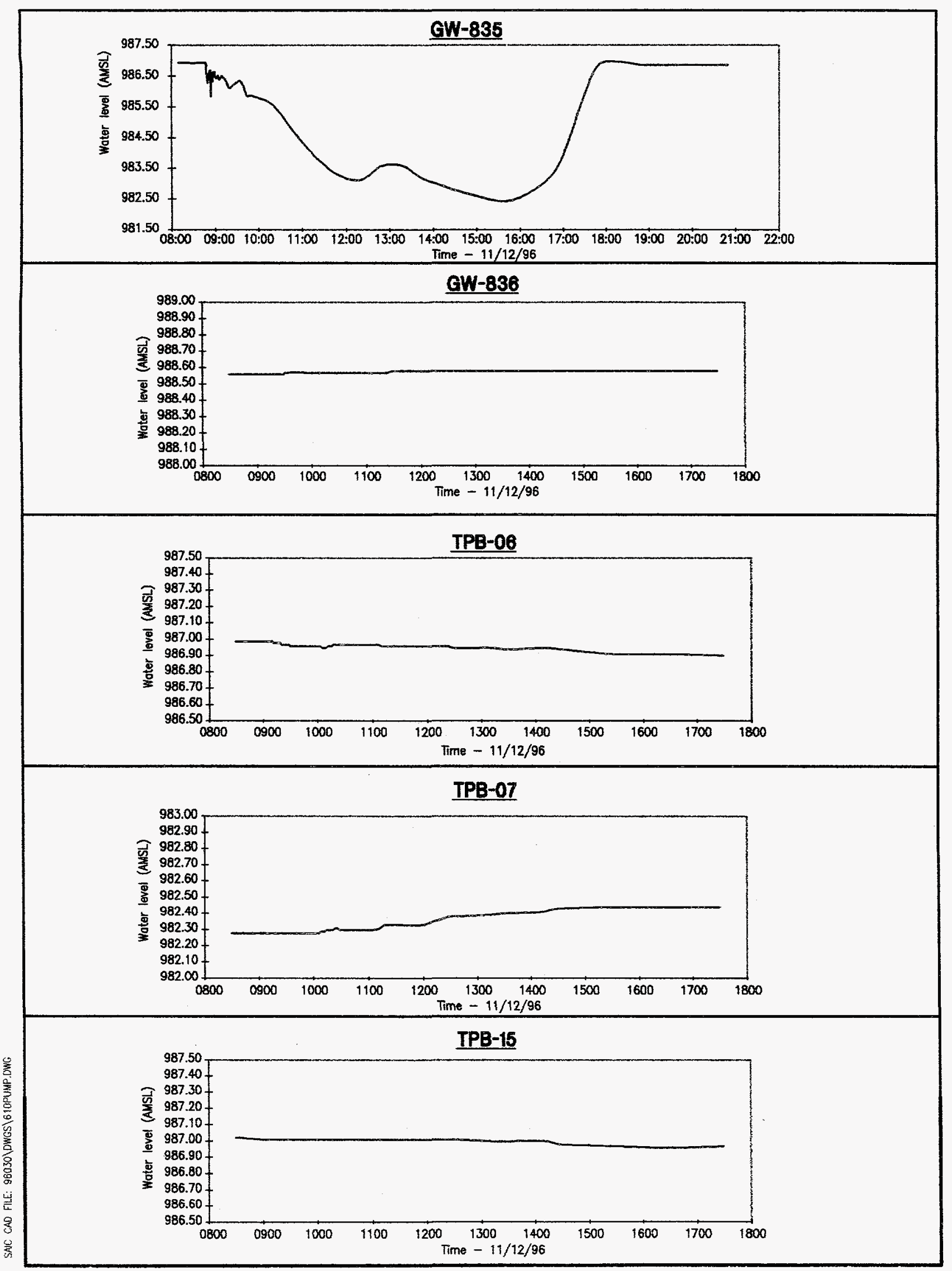

Fig. B.2. Aquifer pumping test water levels in pumped piezometer (GW-835) and observation piezometers (ft-AMSL). 


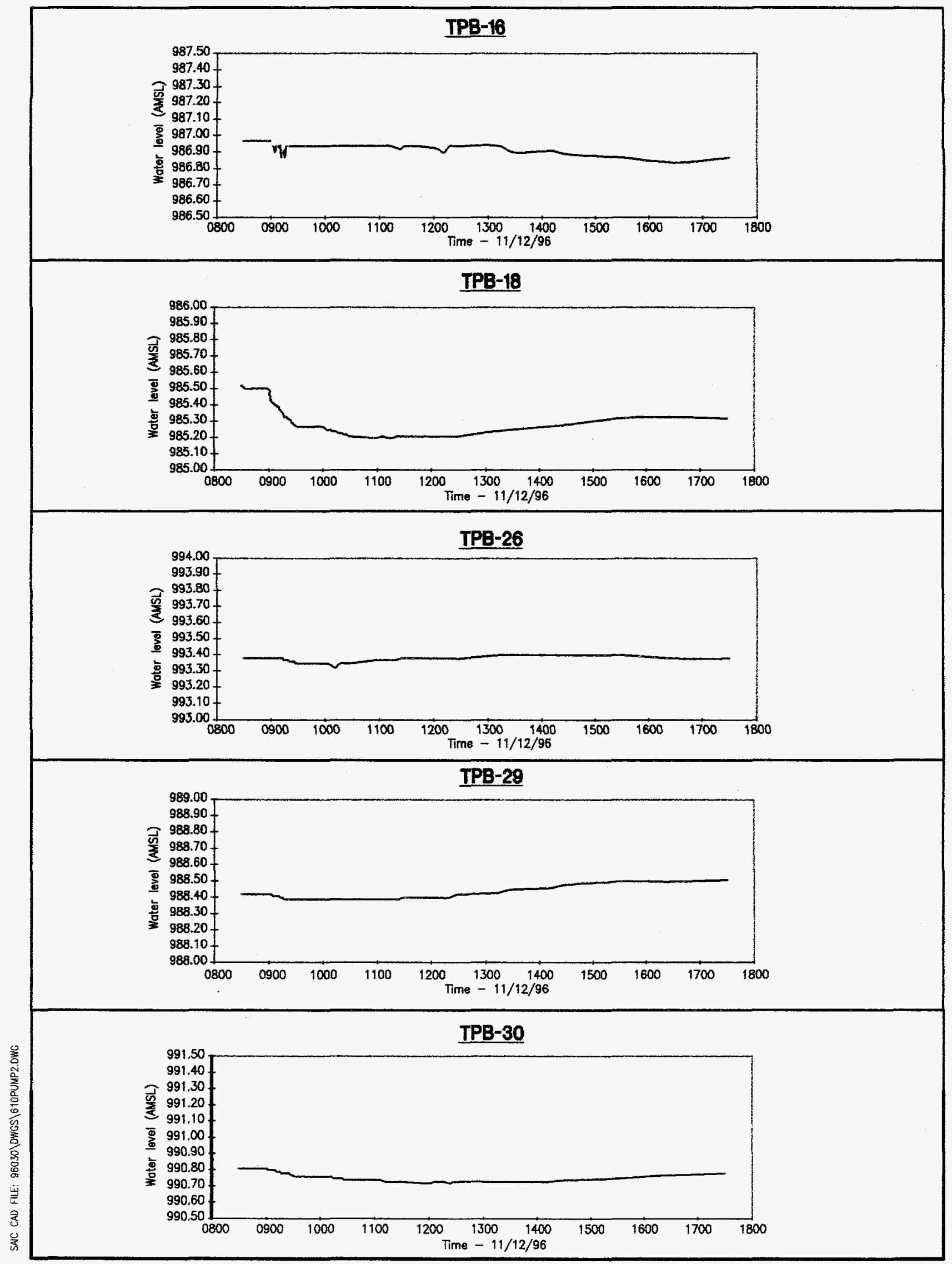

Fig. B.2 (continued) 


\section{APPENDIX C}

SURVEY COORDINATES 



\section{C-3}

Table C.1. Survey coordinates of sample locations Y-12 Plant grid

\begin{tabular}{|c|c|c|c|c|}
\hline Location ID & $\begin{array}{c}\text { East } \\
\text { coordinate }\end{array}$ & $\begin{array}{l}\text { North } \\
\text { coordinate }\end{array}$ & $\begin{array}{l}\text { Surface elevation } \\
\text { (ft-AMSL) }\end{array}$ & $\begin{array}{c}\text { TOC } \\
\text { elevation } \\
\text { (ft-AMSL) }\end{array}$ \\
\hline GW-834 & 50855.00 & 30020.30 & 991.93 & 995.04 \\
\hline GW-835 & 51358.36 & 29822.02 & 997.94 & 1000.91 \\
\hline GW-836 & 51356.24 & 29774.56 & 997.97 & 1001.00 \\
\hline GW-837 & 52281.57 & 29969.06 & 1005.22 & 1007.96 \\
\hline TPB-01 & 50862.26 & 30050.68 & 990.33 & 994.12 \\
\hline TPB-02 & 51002.32 & 30123.76 & 991.72 & 995.00 \\
\hline TPB-04 & 50846.69 & 30017.36 & 990.30 & 992.35 \\
\hline TPB-05 & 50872.25 & 29928.39 & 991.20 & 994.33 \\
\hline TPB-06 & 51410.93 & 29781.15 & 998.17 & 1000.28 \\
\hline TPB-7 & 51229.86 & 29757.29 & 996.63 & 999.48 \\
\hline TPB-8 & 51144.29 & 29745.83 & 988.87 & 991.64 \\
\hline TPB-9 & 52281.32 & 29958.44 & 1004.51 & 1006.78 \\
\hline TPB-10 & 52062.66 & 29911.55 & 1005.04 & 1007.16 \\
\hline TPB-11 & 51838.90 & 29858.65 & 1003.08 & 1005.53 \\
\hline TPB-12 & 49773.83 & 30063.55 & 975.63 & 978.82 \\
\hline TPB-13 & 49551.56 & 29970.97 & 969.94 & 972.73 \\
\hline TPB-14 & 49478.25 & 29912.20 & 971.70 & 973.73 \\
\hline TPB-15 & 51349.84 & 29768.96 & 997.91 & 1000.27 \\
\hline TPB-16 & 51363.03 & 29828.61 & 998.21 & 999.44 \\
\hline TPB-18 & 51286.82 & 29812.18 & 997.30 & 999.97 \\
\hline TPB-19 & 52420.49 & 29948.10 & 1001.36 & 1004.01 \\
\hline TPB-20 & 49702.40 & 30009.87 & 975.75 & 978.33 \\
\hline TPB-21 & 49752.14 & 29940.63 & 980.97 & 983.44 \\
\hline TPB-22 & 49791.72 & 29873.18 & 980.13 & 982.51 \\
\hline TPB-23 & 49471.15 & 29914.63 & 972.76 & 975.00 \\
\hline TPB-24 & 50827.71 & 29965.39 & 991.53 & 994.34 \\
\hline TPB-25 & 52029.77 & 30074.96 & 1007.68 & 1009.03 \\
\hline TPB-26 & 51520.13 & 30151.23 & 1000.36 & 1003.21 \\
\hline TPB-27 & 51237.66 & 30244.31 & 998.72 & 1001.49 \\
\hline TPB-24 & 50827.71 & 29965.39 & 991.53 & 994.34 \\
\hline
\end{tabular}


Table C.1 (continued)

\begin{tabular}{|c|c|c|c|c|}
\hline Location ID & $\begin{array}{c}\text { East } \\
\text { coordinate }\end{array}$ & $\begin{array}{l}\text { North } \\
\text { coordinate }\end{array}$ & $\begin{array}{l}\text { Surface elevation } \\
\text { (ft-AMSL) }\end{array}$ & $\begin{array}{c}\text { TOC } \\
\text { elevation } \\
\text { (ft-AMSL) }\end{array}$ \\
\hline TPB-29 & 51465.49 & 29876.85 & 999.88 & 1003.11 \\
\hline TPB-30 & 51526.39 & 30024.42 & 1000.06 & 1003.72 \\
\hline TPB-31 & 52234.25 & 30052.04 & 1007.42 & 1011.00 \\
\hline TPB-32 & 52236.31 & 30053.34 & 1007.29 & 1010.35 \\
\hline BC-135 & 52261.33 & 29923.70 & 996.53 & NA \\
\hline BC-200 & 52240.08 & 29933.36 & 996.45 & NA \\
\hline BC-300 & 52162.31 & 29907.52 & 995.34 & NA \\
\hline BC- 400 & 52071.93 & 29875.86 & 996.47 & NA \\
\hline BC-500 & 51968.83 & 29838.67 & 995.75 & NA \\
\hline BC- 600 & 51867.33 & 29824.86 & 996.18 & NA \\
\hline BC-700 & 51730.22 & 29835.68 & 993.34 & NA \\
\hline BC-800 & 51664.16 & 29851.77 & 991.97 & NA \\
\hline BC-900 & 51568.14 & 29805.42 & 989.50 & NA \\
\hline $\mathrm{BC}-1000$ & 51486.31 & 29769.34 & 988.55 & NA \\
\hline BC-1030 & 51413.51 & 29756.01 & 986.95 & NA \\
\hline BC-1100 & 51395.34 & 29753.24 & 986.50 & NA \\
\hline BC-1200 & 51278.20 & 29717.51 & 982.42 & NA \\
\hline BC- 1300 & 51175.68 & 29704.18 & 981.37 & NA \\
\hline BC-1400 & 51080.55 & 29706.77 & 978.81 & NA \\
\hline NT $1-000$ & 50684.39 & 29892.57 & 977.70 & NA \\
\hline NT1-075 & 50735.25 & 29964.66 & 977.44 & NA \\
\hline NT1-100 & 50768.49 & 29962.8 & 978.63 & NA \\
\hline NT1-200 & 50813.92 & 30054.54 & 978.90 & NA \\
\hline NT1-300 & 50912.64 & 30105.66 & 980.37 & NA \\
\hline NT1-390 & 50993.64 & 30143.47 & 982.71 & NA \\
\hline NT1-400 & 51002.03 & 30159.45 & 982.65 & NA \\
\hline NT1-500 & 51086.34 & 30222.79 & 985.40 & NA \\
\hline NT1-575 & 51145.17 & 30275.04 & 989.48 & NA \\
\hline NT2-000 & 49204.42 & 29474.25 & 958.46 & NA \\
\hline NT2-100 & 49310.73 & 29519.52 & 959.06 & NA \\
\hline NT2-200 & 49385.56 & 29551.56 & 959.63 & NA \\
\hline
\end{tabular}




$$
\text { C-5 }
$$

Table C.1 (continued)

\begin{tabular}{lcccc}
\hline Location ID & $\begin{array}{c}\text { East } \\
\text { coordinate }\end{array}$ & $\begin{array}{c}\text { North } \\
\text { coordinate }\end{array}$ & $\begin{array}{c}\text { Surface elevation } \\
\text { (ft-AMSL) }\end{array}$ & $\begin{array}{c}\text { TOC } \\
\text { elevation } \\
\text { (ft-AMSL) }\end{array}$ \\
\hline NT2-300 & 49425.94 & 29632.27 & 961.61 & NA \\
NT2-400 & 49412.26 & 29715.58 & 962.51 & NA \\
NT2-500 & 49441.54 & 29794.90 & 962.85 & NA \\
NT2-600 & 49484.70 & 29859.36 & 963.31 & \\
NT2-645 & 49510.40 & 29896.11 & 963.98 & \\
NT2-700 & 49572.13 & 29881.54 & 964.97 & NA \\
NT2-800 & 49607.18 & 29971.78 & 965.29 & NA \\
NT2-900 & 49656.14 & 30045.32 & 967.94 & NA \\
NT2-970 & 49717.68 & 30092.01 & 967.88 & NA \\
NT2-1000 & 49746.42 & 30094.82 & 968.01 & NA \\
NT2-1120 & 49878.94 & 30154.55 & 969.96 & NA \\
\hline
\end{tabular}




\section{DISTRIBUTION}

1. L. V. Asplund

2. W. D. Bostick

3. B. $\mathrm{Gu}$

4. C. S. Haase

5. K. T. Klasson

6. L. Liang

7. H. C. Newsom

8. P. T. Owen

9. L. B. Raulston

10. C. M. Smith

11. A. J. Stewart

12-14. J. H. Vanderlan

15. D. B. Watson

16. File-EMEF DMC-RC

17. R. Adkisson, Entech, 560 Oak Ridge Turnpike, Suite 2, Oak Ridge, Tennessee 37830

18. W. Goldberg, MSE Technology Applications, Inc., P.O. Box 4078, Butte, Montana 59702

19. M. Leavitt, Science Applications International Corporation, 800 Oak Ridge Turnpike, P.O. Box 2502, Oak Ridge, Tennessee 37831

20. D. Moss, Science Applications International Corporation, 800 Oak Ridge Turnpike, P.O. Box 2502, Oak Ridge, Tennessee 37831

21. B. Sams, Jacobs Engineering Group Inc., 125 Broadway Ave., Oak Ridge, Tennessee 37830 


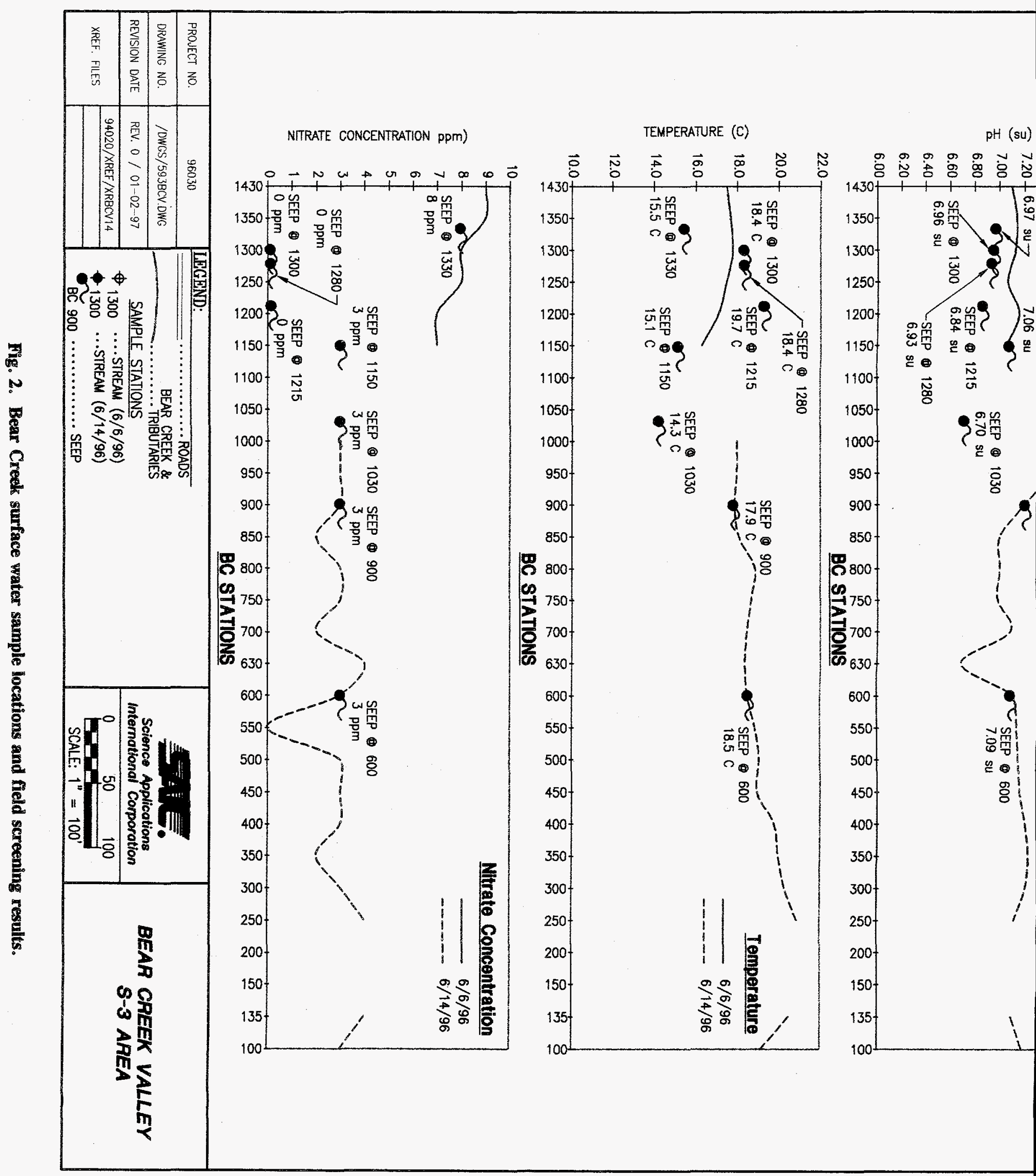

